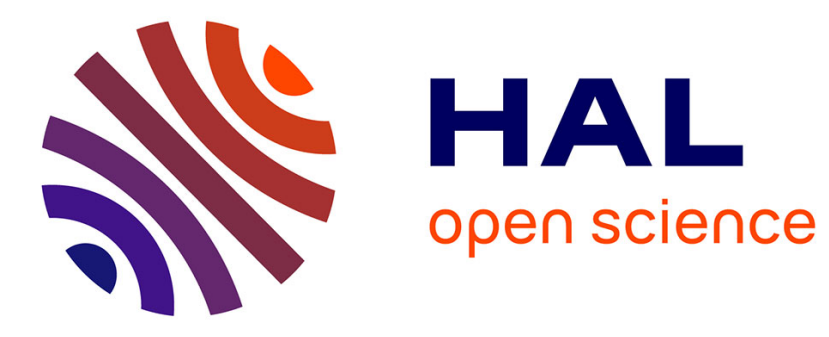

\title{
Large Time Behavior of the Navier-Stokes Flow
}

\author{
Lorenzo Brandolese, Maria E Schonbek
}

\section{To cite this version:}

Lorenzo Brandolese, Maria E Schonbek. Large Time Behavior of the Navier-Stokes Flow. Handbook of Mathematical Analysis in Mechanics of Viscous Fluids, Giga Y., Novotný A. (eds), Springer International Publishing, pp.579-645, 2018. hal-02158208

\section{HAL Id: hal-02158208 \\ https://hal.science/hal-02158208}

Submitted on 17 Jun 2019

HAL is a multi-disciplinary open access archive for the deposit and dissemination of scientific research documents, whether they are published or not. The documents may come from teaching and research institutions in France or abroad, or from public or private research centers.
L'archive ouverte pluridisciplinaire HAL, est destinée au dépôt et à la diffusion de documents scientifiques de niveau recherche, publiés ou non, émanant des établissements d'enseignement et de recherche français ou étrangers, des laboratoires publics ou privés. 


\title{
Large time behavior of the Navier-Stokes flow*
}

\author{
Lorenzo Brandolese ${ }^{\dagger \ddagger}$ and Maria E. Schonbek ${ }^{\S}$
}

August 23, 2016

\begin{abstract}
Different results related to the asymptotic behavior of incompressible fluid equations are analyzed as time tends to infinity. The main focus is on the solutions to the Navier-Stokes equations, but in the final section a brief discussion is added on solutions to Magneto-Hydrodynamics, Liquid crystals, Quasi-Geostrophic and Boussinesq equations. Consideration is given to results on decay, asymptotic profiles, and stability for finite and nonfinite energy solutions.
\end{abstract}

\section{Introduction}

This chapter focuses on the asymptotic behavior of solutions to dissipative incompressible fluid equations. The diffusion for these systems is given by the presence of a Laplacian or a fractional Laplacian. When a stationary solution exists, an important issue is to establish if as time goes to infinity, the evolutionary solutions tend to the corresponding stationary ones. The question at hand then, is to investigate the asymptotic stability of the stationary solutions. If the original system has no external forcing terms, it is surmised that the stationary solution is zero. The stability is analyzed in $L^{p}\left(\mathbb{R}^{n}\right), p \geq 1$, Sobolev, Lorentz and Besov spaces. Emphasis is on solutions to the incompressible Navier-Stokes equations. In the last section of the chapter, brief consideration is given to the decay of solutions corresponding to the Quasi-Geostrophic equations, Magneto-Hydrodynamics, Boussinesq, and to Liquid Crystal systems. This small sample of "Navier-Stokes like" equations illustrates how the methods developed in the study of large time behavior of basic fluid motions can be adapted to more complicated models.

Asymptotic behavior of Leray's weak solutions to the Navier-Stokes equations in $\mathbb{R}^{n}$ is the central theme of the first part of this chapter. Upper and lower bounds of rates

\footnotetext{
*In: Handbook of Mathematical Analysis in Mechanics of Viscous Fluids. Part I. Incompressible fluids. Unsteady viscous Newtonian fluids. Yoshikazu Giga and Antonn Novotný editors. Springer

${ }^{\dagger}$ Université de Lyon, Université Lyon 1. CNRS UMR 5208 Institut Camille Jordan, 43 bd. du 11 novembre, Villeurbanne Cedex F-69622, France. E-mail: brandolese@math.univ-lyon1.fr

${ }^{\ddagger} \mathrm{L}$. Brandolese is supported by the ANR project DYFICOLTI N. 36338

${ }^{\S}$ Department of Mathematics, UC Santa Cruz, Santa Cruz, CA 95064, USA. E-mail: schonbek@math.ucsc.edu
} 
of decay of the solutions are obtained, mostly when the corresponding system has zero external forces. The aim is to obtain optimal rates of decay, i.e. rates that coincide with the corresponding solutions of the underlying linear part: this is often achieved by establishing that the difference between the solution and its linear counterpart decays faster than the latter. Consideration is then given to several classes of strong solutions for which more detailed information can be obtained: point-wise decay, asymptotic profiles, etc.

Once finite energy solutions have been investigated, various scenarios involving non finite $L^{2}$-norms for the Navier-Stokes flows are discussed. Restricting attention to finite energy solutions seems perfectly natural looking at the energy inequality, but there are some drawbacks, it does not cover for instance: the dynamics of flows with vorticity $\omega$ in $L^{1}\left(\mathbb{R}^{2}\right)$. This situation has recently attracted considerable interest. These are typically infinite energy flows, as the $L^{2}$ condition for the velocity field would otherwise force the corresponding vorticity to have zero mean. The reader will find more on this topic in Th. Gallay and Y. Maekawa's contribution [84] of the present handbook.

A second drawback appears looking at the scale invariance of the Navier-Stokes equations: $u \mapsto u_{\lambda}$, where $u_{\lambda}(x, t)=\lambda u\left(\lambda x, \lambda^{2} t\right)$. The function space associated with the energy inequality is $X=L^{2}\left((0, \infty), L^{2}\left(\mathbb{R}^{n}\right)\right) \cap L^{2}\left((0, \infty), \dot{H}^{1}\left(\mathbb{R}^{n}\right)\right)$, but $\left\|u_{\lambda}\right\|_{X}$ is independent of $\lambda$ only in dimension 2. Starting with the classical contributions of H. Fujita and T. Kato [75], [122] a large amount of research has been developed regarding the constructions of solutions in function spaces respecting the scale invariance. These solutions in general have infinite energy. As soon as $u_{0}$ satisfies a suitable smallness condition, (that can be dropped in the 2D case), the solutions are global-in-time. The construction of such solutions often provides valuable information on their decay as $t \rightarrow \infty$. I. Gallagher's [82] contribution in this handbook presents a detailed account on well-posedness issues in scale invariant spaces. Here the focus is only on a few aspects of the large time decay results that follow from the so-called Kato's method.

A closely related issue to the scaling invariance is self-similarity. That is, solutions of the Navier-Stokes equations, like those constructed by Y. Giga and T. Miyakawa [96], or by M. Cannone, Y. Meyer and F. Planchon [37]. Such solutions that are left-invariant by the natural scaling, $u=u_{\lambda}$, play a major role, e.g., in the description of the large time behavior of flows that are asymptotically homogeneous, in the study of stability of stationary flows, in the understanding of axisymmetric flows, etc. Results on self-similar profiles are discussed on brief terms only as the reader will find a detailed account in H. Jia, V. Šverák and T.-P. Tsai's contribution [116] to this handbook.

In Section 3 attention is turned on large time decay results for Navier-Stokes flows in domains other than the whole $\mathbb{R}^{n}$. The presentation of this topic will be more succinct than in the case of the whole space. In particular, the focus is on the classical cases of bounded or exterior domains with smooth boundaries.

In Section 4 results are reviewed on the asymptotic stability of stationary solutions. Recent developments on this topic are discussed: For certain solutions to the Navier-Stokes 
equations (possibly of infinite energy) results are presented that analyze the stability under arbitrary large $L^{2}$ perturbations. This is the case, for instance, of stationary solutions associated with a time-independent external force, suitably small in some rough norm. The classical one-parameter family of Landau solutions are asymptotically stable in this sense, at least when the parameter belongs to an appropriate range. In particular, results from [119] and its extensions in $[111,120]$, that deal with the asymptotics of such flows are discussed.

The last section considers decay for other diffusive models. Among them, the QuasiGeostrophic equations which are an interesting example, of a simplified model arising in geophysics, with a diffusive term given by a fractional power of the Laplacian. The other models discussed (MHD, inhomogeneous NS, Boussinesq system, Nematic liquid crystals equations) require a more involved analysis since for them the Navier-Stokes system is strongly coupled with one or more additional equations.

\section{Long-time behavior for the Navier-Stokes equations in the whole space}

\subsection{The energy decay problem of weak solutions}

The first part of this chapter is centered on solutions to the Cauchy problem for the incompressible Navier-Stokes equations:

$$
\begin{aligned}
u_{t}-\Delta u+(u \cdot \nabla) u+\nabla p & =f, \quad(x, t) \in \mathbb{R}^{n} \times(0, \infty), n \geq 2, \\
\operatorname{div} u & =0 \\
u(x, 0) & =u_{0}(x)
\end{aligned}
$$

As usual, $u=u(x, t)$ represents the velocity of the fluid, $p=p(x, t)$ is the pressure and, $f=f(x, t)$ is the external force. The classical theory of viscous, incompressible fluid flows is governed by these equations. A large area of modern research is devoted to deducing the qualitative behavior of their solutions. The results on existence and asymptotic behavior depend on the initial conditions and, either boundary requirements or conditions when $|x| \rightarrow \infty$. Also adequate constraints need to be stipulated for the forcing term $f$. For simplicity, the viscosity coefficient is set equal to one, as this can be always achieved by an appropriate rescaling. All the results remain valid in the presence of a coefficient $\nu>0$ in the diffusive term. What will change is the dependence on $\nu$ for estimates. In the sequel the Navier-Stokes equations are referred to by NS. The literature related to the NS equations is too vast to attempt a complete list of references. The results in this chapter are restricted to the analysis related to the asymptotic behavior.

The closing remark of the 1934 pioneering paper on the NS equations by J. Leray [145] (reproduced in the figure above), where global solutions were constructed for the first time, states:

"N.B. I do not know if $W(t)$ goes necessarily to 0 when $t$ grows indefinitely." 


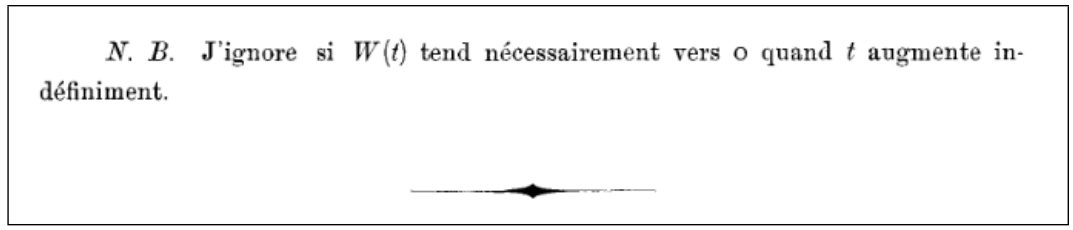

Here $W(t)$ refers to the $L^{2}\left(\mathbb{R}^{n}\right)$-norm of the weak solution to NS that Leray constructed, under the only assumption that $u_{0}$ is in $L^{2}\left(\mathbb{R}^{n}\right)$ and divergence-free. The question in the $N$.B. was addressed in the particular case $f \equiv 0$ and for $n=3$. Since then, this question has been studied not only for the decay in $L^{2}\left(\mathbb{R}^{3}\right)$, but for many other spaces and, non zero forcing terms.

\subsection{Small data approach and T. Kato's decay results}

The first answer to Leray's Nota Bene stated above, was given by T. Kato ([122, Theorem $\left.4^{\prime}\right]$ ), showing decay to zero of solutions with datum in $L^{2}\left(\mathbb{R}^{3}\right)$ and zero forcing term. The results in [122] are very deep and helped to lay the groundwork for decay and existence questions for the solutions to the Navier-Stokes equations.

Before stating the results from [122] the following notation is recalled: (note that the notations used will not distinguish between function space of scalar and vector fields,)

1. $L_{\sigma}^{2}=L_{\sigma}^{2}\left(\mathbb{R}^{n}\right)=\left\{v \in L^{2}\left(\mathbb{R}^{n}\right): \operatorname{div} v=0\right\}$, where the divergence of the vector field $v$ is taken in the distributional sense.

2. $\mathbb{P}$ denotes the Leray projector, the orthogonal projector mapping $L^{2}$ onto $L_{\sigma}^{2}$. This projector extends to more general spaces, including the $L^{p}$ spaces, $1<p<\infty$.

3. $\mathbb{P} L^{n}=\left\{v \in L^{n}\left(\mathbb{R}^{n} ; \mathbb{R}^{n}\right), \operatorname{div} v=0\right\}$.

Once local existence of solutions with datum in $\mathbb{P} L^{n}$ had been established in [122], it is shown that they can be extended globally, provided the datum is small in $L^{n}\left(\mathbb{R}^{n}\right)$. Next the decay in $L^{q}\left(\mathbb{R}^{n}\right)$ spaces is analyzed for the solution and the first derivatives. The decay rates obtained coincide with the rates for solutions to the underlying Stokes flow. The two main results in this paper state:

Theorem 2.1 ([122]). Let $u_{0} \in \mathbb{P} L^{n}$. Then there is $T>0$ and a unique solution such that

$$
\begin{gathered}
t^{\frac{1}{2}-\frac{n}{2 q}} u \in B C\left([0, T) ; \mathbb{P} L^{q}\right), \quad n \leq q \leq \infty, \\
t^{1-\frac{n}{2 q}} D u \in B C\left([0, T) ; \mathbb{P} L^{q}\right), \quad n \leq q<\infty .
\end{gathered}
$$

The notation $B C$ refers to bounded continuous functions. The $L^{p}$-norms will be denoted simply by $\|\cdot\|_{p}$. 
If $\left\|u_{0}\right\|_{n}<\lambda$ with $\lambda$ sufficiently small, then the time $T$ from the above theorem can be taken to be infinity and the following rates of decay are valid:

$$
\|u\|_{q} \leq C t^{-\frac{1}{2}+\frac{n}{2 q}}, n \leq q \leq \infty, \quad\|D u\|_{q} \leq C t^{-\left(1-\frac{n}{2 q}\right)}, n \leq q<\infty .
$$

Furthermore, Kato also established the following variant for data with better integrability properties:

Theorem 2.2 ([122]). Let the initial data $u_{0} \in \mathbb{P} L^{n} \cap L^{p}, 1<p<n$. There exists $\lambda>0$ such that if $\left\|u_{0}\right\|_{n} \leq \lambda$, then the solution constructed in Theorem 2.1 is global. Moreover for any finite $q \geq p$

$$
t^{\frac{n}{2}\left(\frac{1}{p}-\frac{1}{q}\right)} u \in B C\left([1, \infty) ; \mathbb{P} L^{q}\right), \quad t^{\left.\frac{n}{2}\left(\frac{1}{p}-\frac{1}{q}\right)+1\right)} D u \in B C\left([1, \infty) ; \mathbb{P} L^{q}\right),
$$

provided the exponents of $t$ are smaller then 1, otherwise they be should replaced by an arbitrary number smaller then 1 . Moreover, $\|u(t)\|_{p} \rightarrow 0$ as $t \rightarrow \infty$.

The proof of these theorems is based on the construction of a sequence of approximating solutions represented in the mild form. Recursive bounds combined with a passage to the limit in this sequence yield the decay rates. For the argument to work, Kato needs at each step a uniform bound which is only possible if either the data or the time of existence are sufficiently small. The results in [122] can easily be extended to solution with non zero forcing terms with appropriate decay conditions. A short sketch of Kato's arguments are presented here.

Sketch of Kato's proof. Rewrite the NS solution as

$$
u_{t}+A u+\mathbb{P} \nabla \cdot(u \otimes u)=0
$$

where $A u=-\mathbb{P} \Delta u$. In the whole space, the projector $\mathbb{P}$ commutes with the Laplacian and, $A$ boils down to the operator $-\Delta$ when applied to divergence-free vector fields. The solution in mild form can be represented by:

$$
u=e^{-t A} u_{0}+G u, \quad \text { with } \quad G u(t)=-\int_{0}^{t} e^{-(t-s) A} \mathbb{P} \nabla \cdot(u \otimes u)(s) \mathrm{d} s .
$$

Let $\alpha, \beta, \gamma>0$ and $\gamma \leq \alpha+\beta<m$, then

$$
\left\|D^{\mu} G u(t)\right\|_{\frac{n}{\gamma}} \leq C \int_{0}^{t}(t-s)^{-\frac{1}{2}(\mu+\alpha+\beta-\gamma)}\|u\|_{\frac{n}{\gamma}}\|D u\|_{\frac{n}{\beta}} \mathrm{d} s, \quad \mu=0,1 .
$$

Construct a sequence of the form

$$
u_{0}=u(x, 0), \quad u_{m+1}=a_{0}+G u_{m}
$$

This allows to obtain the inductive estimates, for any $0<\delta<1$,

$$
t^{\frac{1}{2}(1-\delta)} u_{m} \in B C\left([0, \infty] ; \mathbb{P} L^{\frac{n}{\delta}}\right), \quad\left\|u_{m}\right\|_{\frac{n}{\delta}} \leq K_{m}
$$




$$
t^{\frac{1}{2}} D u_{m} \in B C\left([0, \infty] ; \mathbb{P} L^{\frac{n}{\delta}}\right),\left\|u_{m}\right\|_{\frac{n}{\delta}} \leq K_{m}^{\prime}
$$

Setting

$$
K_{0}=K_{0}^{\prime}=C\left\|u_{0}\right\|_{n}
$$

it is possible to show that

$$
K_{m+1} \leq K_{0}+C K_{m} K_{m}^{\prime}, \quad K_{m+1}^{\prime} \leq K_{0}^{\prime}+C K_{m} K_{m}^{\prime}
$$

The last estimates for a small time interval or, for data with sufficiently small norm yield $K_{0}^{\prime}+C K_{m} K_{m}^{\prime} \leq K$, with $K$ a fixed constant. If $n \leq q \leq \infty, \gamma=\frac{n}{q}, \alpha=\delta$ and $\beta=1$ it follows that

$$
\left\|u_{m+1}\right\|_{q} \leq\left\|e^{-t A} u_{0}\right\|_{q}+C K_{m} K_{m}^{\prime} \int_{0}^{t}(t-s)^{-\frac{1}{2}\left(1+\delta-\frac{n}{q}\right)} s^{-\left(1-\frac{\delta}{2}\right)} \mathrm{d} s \leq K t^{-\frac{1}{2}\left(1-\frac{n}{q}\right)} .
$$

Passing to the limit as $m \rightarrow \infty$ gives the conclusion of the theorem.

The last claim of the second Theorem, applied to $n=3$ and $p=2$, answers Leray's questions, at least for small solutions. Kato concludes his paper by showing how his results, in fact, apply to Leray's weak solutions, that eventually become small, at least in dimension $\leq 4$.

\subsection{Masuda's approach and nonuniform decay}

The same year that Kato's decay results were established, K. Masuda [156] proved the non-uniform decay for weak solutions with large data in $L^{2}\left(\mathbb{R}^{n}\right)$ and appropriate non zero forcing terms. His method applies to more general domains. Before stating Masuda's main result, it is recalled tha, by definition, a weak solution to NS is a function $u \in$ $C_{w}\left([0, \infty], L^{2}\left(\mathbb{R}^{n}\right)\right) \cap L^{2}\left(\mathbb{R}_{+}, \dot{H}^{1}\left(\mathbb{R}^{n}\right)\right)$, which satisfies

$$
\begin{aligned}
\langle u(t), \phi(t)\rangle & +\int_{0}^{t}\left\{\left\langle u(s), \frac{\partial \phi}{\partial s}\right\rangle+\langle\nabla u(s), \nabla \phi(s)\rangle+\langle(u(s) \cdot \nabla) u(s), \phi(s)\rangle\right\} \mathrm{d} s \\
& =\int_{0}^{t}\langle f(s), \phi(s)\rangle d s+\left\langle u_{0}, \phi(0)\right\rangle, \quad t>0,
\end{aligned}
$$

for all $\phi \in C\left([0, \infty), C_{\sigma}^{\infty}\left(\mathbb{R}^{n}\right)\right)$, where $C_{\sigma}^{\infty}\left(\mathbb{R}^{n}\right)$ is the set for smooth and compactly supported functions with zero divergence. Here $\langle\cdot, \cdot\rangle$ denotes the $L^{2}$-inner product.

For $u_{0} \in L^{2}\left(\mathbb{R}^{n}\right)$ and all $f \in L_{l o c}^{1}\left(\mathbb{R}^{+}, L^{2}\left(\mathbb{R}^{2}\right)\right)$ a weak solution can be constructed, in an arbitrarily large time interval $(0, T)$, satisfying the energy inequality:

$$
\|u(t)\|_{2}^{2}+2 \int_{0}^{t}\|\nabla u(r)\|_{2}^{2} \mathrm{~d} r \leq\left\|u_{0}\right\|_{2}^{2}+2 \int_{0}^{t}\langle f, u\rangle \mathrm{d} r, \quad \text { for all } t \geq 0 .
$$

On the other hand, the so called "strong energy inequality" is the variant of (4) that reads

$$
\|u(t)\|_{2}^{2}+2 \int_{s}^{t}\|\nabla u\|_{2}^{2} \leq\|u(s)\|_{2}^{2}+2 \int_{s}^{t}\langle f, u\rangle, \quad \text { for } s=0, \text { almost all } s>0 \text { and all } t \geq s .
$$


Inequality (5) is only known to be true in dimensions $n=2,3,4$ (or in any dimension, but for bounded domains). When available, such inequality provides useful additional information on the energy. Next, Masuda's result is stated

Theorem $2.3([156])$. Let $u_{0} \in L_{\sigma}^{2}\left(\mathbb{R}^{n}\right)$, and the forcing term $f$ with the projection Pf $\in L^{1}\left(\mathbb{R}^{+}, L^{2}\left(\mathbb{R}^{2}\right)\right)$. Let $u$ be a weak solution to the Navier-Stokes equations in $\mathbb{R}^{n}$ such that $\int_{0}^{\infty}\|\nabla u\|_{2}^{2}<\infty$. Then

$$
\int_{t}^{t+1}\|u(s)\|_{2}^{2} d s \rightarrow 0, \quad \text { as } t \rightarrow \infty
$$

In particular, if $\|u(t)\|_{2}$ is monotonically decreasing for large enough $t$ (when $f=0$, this is the case at least for the weak solutions constructed by Leray in the case $n=3$, and those obtained by Leray's method for $2 \leq n \leq 4)$ then $\|u(t)\|_{2} \rightarrow 0$.

Ideas from Masuda's proof. The first step consists in showing that $\lim _{t \rightarrow \infty}\left\|(I+A)^{-\alpha} u\right\|_{2}=$ 0 . Here $A=-\mathbb{P} \Delta$ and $\alpha=(n-2) / 4$. The crucial estimate of Masuda's proof follows from an interpolation inequality and reads, with $\beta=1 /(1+2 \alpha)$,

$$
\int_{t}^{t+1}\|u(s)\|_{2}^{2} \leq\left(\int_{t}^{t+1}\left\|(I+A)^{-\alpha} u(s)\right\|_{2}^{2}\right)^{\beta}\left(\int_{t}^{t+1}\left\|(I+A)^{1-\beta} u(s)\right\|_{2}^{2}\right)^{1-\beta} .
$$

The second integral on the right turns out to be bounded. This is used to obtain that $\lim _{t \rightarrow \infty} \int_{t}^{t+1}\|u(s)\|_{2}^{2} \mathrm{~d} s=0$. The decay is then immediate, since, when the $L^{2}$-norm is decreasing, it converges to a constant, and this constant must be zero by the above integral limit.

Masuda's result that $\|u(t)\|_{2} \rightarrow 0$ not only applies to Leray's solutions, but also to a class of weak solutions constructed by O. Ladyzhenskaya [141], whose energy decays monotonically for large enough times.

For data exclusively in $L_{\sigma}^{2}$, decay without a uniform rate is the best that can be expected. Even at the level of the underlying linear part, solutions to the heat equations, with data in $L^{2}$ alone, cannot decay at a uniform algebraic rate. For such solutions, this can be seen considering the rescaled data $u_{0}^{\alpha}(x)=\alpha^{\frac{n}{2}} u_{0}(\alpha x)$. Such initial values have all the same $L^{2}$-norm, and it is easy to show that, for all $T>0$,

$$
\lim _{\alpha \rightarrow 0} \frac{\left\|e^{T \Delta} u_{0}^{\alpha}\right\|_{2}}{\left\|u_{0}^{\alpha}\right\|_{2}}=1
$$

Thus, no better decay rates can be expected for solutions to the Navier-Stokes equations. Specifically for any $\beta>0, \epsilon>0$ and $T>0$ one can find (see [181]) $u_{0}$ such that $\left\|u_{0}\right\|_{2}=\beta$ and

$$
\frac{\|u(T)\|_{2}}{\left\|u_{0}\right\|_{2}} \geq 1-\epsilon
$$

For exterior domains the lack of decay uniformity for $L_{\sigma}^{2}$ was established by Hishida in [112]. 


\subsection{Fourier splitting method and decay rates of weak solutions}

For weak solutions to the NS equations, M.E. Schonbek, [180], obtained algebraic decay for the Cauchy problem with large datum in $L^{1}\left(\mathbb{R}^{n}\right) \cap L^{2}\left(\mathbb{R}^{n}\right), n \geq 3$ and $f=0$. The Fourier splitting method used in [180], was first introduced to obtain the algebraic rates of decay to parabolic conservation laws [179]. Moreover this method serves for a wide class of diffusive systems that satisfy an appropriate integral energy inequality. The method is based on the fact that the low frequencies of the data plays a significant role in the decay. This fact will be discussed in more detail in Section 2.12. In the sequel Fourier splitting method will be referred by FS.

A brief formal description of the FS technique is given following the steps in [183]. For a rigorous proof, FS can be applied to a sequence of approximating solutions and the decay follows by passing to the limit. Specifically FS is a technique used to determine the decay rates of solutions to an integral differential energy inequality of the form

$$
\frac{\mathrm{d}}{\mathrm{d} t} \int_{\mathbb{R}^{n}}|u|^{2} \mathrm{~d} x \leq-C_{0} \int_{\mathbb{R}^{n}}\left|D^{m} u\right|^{2} \mathrm{~d} x, \quad x \in \mathbb{R}^{n}, t>0
$$

where $C_{0}>0$ and $m>0$ are independent on $u$ and on $t$. (Note that when $m=0$, exponential decay follows). In the sequel $\hat{v}(\xi)$ denotes the Fourier transform of $v$. For the FS method the following set $\mathcal{S}_{m}=\mathcal{S}_{m}(t)$ needs to be defined:

$$
\mathcal{S}_{m}=\left\{\xi \in \mathbb{R}^{n}:|\xi| \leq\left(\frac{n}{C_{0}(t+1)}\right)^{\frac{1}{2 m}}\right\} .
$$

Theorem 2.4. Let $u=u(x, t)$ satisfy the integral differential inequality (6), let $u_{0} \in$ $L^{2}\left(\mathbb{R}^{n}\right)$. If there exists $r>0$ and $\mathcal{A}>0$ so that $|\hat{u}(\xi, t)| \leq \mathcal{A}$ for all $|\xi|<r$, then for some constant $K>0$ depending on $r, \mathcal{A}$ and the $L^{2}$ data of the solution, it follows that

1. If $1<2 m \Rightarrow\|u(t)\|_{2}^{2} \leq K(t+1)^{-\frac{n}{2 m}}$.

2. If $1=2 m \Rightarrow\|u(t)\|_{2}^{2} \leq K(t+1)^{-n} \ln (t+1)$.

3. If $1>2 m \Rightarrow\|u(t)\|_{2}^{2} \leq K(t+1)^{-n}$.

Sketch of the formal proof. By Plancherel's theorem inequality (6) can be rewritten as

$$
\frac{\mathrm{d}}{\mathrm{d} t} \int_{\mathbb{R}^{n}}|\hat{u}|^{2} \mathrm{~d} \xi \leq-C_{0} \int_{\mathbb{R}^{n}}|\xi|^{2 m}|\hat{u}|^{2} \mathrm{~d} \xi
$$

Split the frequency space on the right hand side of the last inequality into integrals over $\mathcal{S}_{m}$ and $\mathcal{S}_{m}^{c}$, drop the term that comes from the low frequencies:

$$
\frac{\mathrm{d}}{\mathrm{d} t} \int_{\mathbb{R}^{n}}|\hat{u}|^{2} \mathrm{~d} \xi \leq-\frac{n}{t+1} \int_{\mathcal{S}_{m}^{c}}|\hat{u}|^{2} \mathrm{~d} \xi=-\frac{n}{t+1} \int_{\mathbb{R}^{n}}|\hat{u}|^{2} d \xi+\frac{n}{t+1} \int_{\mathcal{S}_{m}}|\hat{u}|^{2} \mathrm{~d} \xi .
$$

Note that $t$ can be taken as large as necessary so that for all $|\xi| \in \mathcal{S}_{m}$ one has $|\xi|<r$. Hence, by the pointwise bound assumption on $\hat{u}(\cdot, t)$, it follows that

$$
\frac{\mathrm{d}}{\mathrm{d} t}\left[(t+1)^{n} \int_{\mathbb{R}^{n}}|u|^{2} \mathrm{~d} x\right] \leq C_{n}(t+1)^{n-1-\frac{n}{2 m}}
$$


Integrating in time the last inequality yields the conclusion of the theorem.

FS can also be applied for differential inequalities of the form

$$
\frac{d}{d t} \int_{\mathbb{R}^{n}}|u|^{2} d x \leq-C \int_{\mathbb{R}^{n}}\left|D^{m} u\right|^{2} d x+g(t), \quad t>0,
$$

for appropriate functions $g(t)$. The technique can be used for solutions to systems such as Navier-Stokes, Magneto-Hydrodynamics, and several other diffusive equations. In the particular case of the NS and MHD equations, inequality (7) if there is no external force, will be satisfied with $m=1$ and $g(t)=0$. For estimates of higher-order derivatives and/or solutions of systems with non-vanishing forcing terms, non zero functions $g(t)$ will appear, see $[184,190]$. Other diffusive systems may involve different values of $m$ and $g(t)$. See Section 5.4 for applications of FS to fractional diffusion operators.

Ideas for the formal decay proof of solutions $(u, p)$ to $N S$. The above FS argument is completed by suitable point-wise estimates on the Fourier Transform $\widehat{u}$. Namely, using

$$
|\widehat{u \cdot \nabla u}|+|\widehat{\nabla p}| \leq C|\xi||\widehat{u u}| \leq C|\xi|\|u(t)\|_{2}^{2}
$$

one gets

$$
|\hat{u}(\xi, t)| \leq e^{-|\xi|^{2} t}\left|\hat{u}_{0}\right|+c \int_{0}^{t} e^{-|\xi|^{2}(t-s)}|\xi|\|u(s)\|_{2}^{2} \mathrm{~d} s
$$

In [180] for integrable initial data, the point-wise bound for $|\hat{u}(\xi, t)| \leq C|\xi|^{-1}$ was then obtained, yielding the non-optimal decay rate $\|u(t)\|_{2}^{2} \leq C(t+1)^{-\frac{n-1}{2}}$. After this preliminary decay result, better rates (assuming $u_{0} \in L^{p} \cap L^{2}\left(\mathbb{R}^{n}\right)$, for some $1 \leq p<2$ ) were obtained by R. Kajikiya and T. Miyakawa [118] for dimensions $n \geq 2$. The improvement in [118] was based on using spectral theory for self adjoint operators. For dimensions $n \geq 3$ optimal decay rates were also obtained by M.E. Schonbek [181], by combining the FS method with improved frequency estimates. In two dimensions, the FS-method as used in [180] only gave logarithmic decay. Its variant proposed by M. Wiegner [205], consisting in applying the Fourier splitting argument to the integral equation, gave the optimal decay for dimensions $n \geq 2$. Furthemore, Wiegner's result extends to give uniform decay rates for solutions arising from initial data $u_{0}$ that do not need to belong to any $L^{p}$-space, with $p \neq 2$, but for which the decay of the heat equation is prescribed.

Notation and preliminaries, that will be needed, are recalled before giving the main results in [205]. Wiegner assumed the initial data $u_{0}$ and the external force $f$ belong to the following class of functions:

$$
\mathcal{D}_{\alpha}^{n}=\left\{\left(u_{0}, f\right) \in \mathcal{L}:\left\|u_{0}(t)\right\|_{2}^{2}+(1+t)^{2}\|f(t)\|_{2}^{2} \leq C(1+t)^{-\alpha} \text {, some } C>0, \alpha>0\right\},
$$

where $\mathcal{L}=L^{2}\left(\mathbb{R}^{n}\right)^{n} \times L^{1}\left(\mathbb{R}_{+},\left(\mathbb{R}^{n}\right)^{n}\right)$ and $u_{0}(t)$ is the solution to the heat equation with data $u_{0}$ and forcing term $f$. The main results of [205] are described in the following Theorem 
Theorem 2.5 ([205]). Let $n \geq 2$. Let $u$ be a weak solution to the NS equations with data and forcing terms $\left(u_{0}, f\right)$. Suppose the strong energy inequality (5) holds. Let $\left(u_{0}, f\right) \in \mathcal{D}_{\alpha}^{n}$. Then

1. $\|u(t)\|_{2}^{2} \leq C(1+t)^{-\bar{\alpha}}$, with $\bar{\alpha}=\min \left\{\alpha, \frac{n}{2}+1\right\}$

2. $\left\|u(t)-u_{0}(t)\right\|_{2}^{2} \leq h_{\alpha}(t)(1+t)^{-d}$, where $d=\frac{n}{2}+1-2 \max \{1-\alpha, 0\}$, and

$$
h_{\alpha}(t)=\left\{\begin{array}{l}
\epsilon(t) \text { for } \alpha=0 \text { with } \epsilon(t) \searrow 0, \\
C \ln ^{2}(1+\epsilon(t)) \quad \text { for } \alpha=1, \\
C \text { for } \alpha \neq 0,1
\end{array} \quad \text { as } t \rightarrow+\infty .\right.
$$

As pointed out in [205], the assumption on the validity of the strong energy inequality (5) can be relaxed by requiring that weak solutions are suitably approximated by sequences of solutions verifying such inequality. Since this is known to be true also when $n \geq 5$, Wiegner's decay result applies in any dimension.

Remark 2.6. If $u_{0} \in L^{1} \cap L_{\sigma}^{2}$, then $\int u_{0}=0$, see [160]. If one additionally assumes that $\int(1+|x|)\left|u_{0}(x)\right| \mathrm{d} x<+\infty$, then the solution of the heat equation satisfies $\left\|u_{0}(t)\right\|_{2}=$ $\mathcal{O}\left(t^{-(n+2) / 4}\right)$ as $t \rightarrow+\infty$ (see [161]). By Wiegner's theorem applied with $f \equiv 0$, one can construct a weak solution arising from $u_{0}$ such that $\|u(t)\|_{2}=\mathcal{O}\left(t^{-(n+2) / 4}\right)$ as $t \rightarrow+\infty$. As observed above, this is true in any space dimension $n \geq 2$. As it will be seen in the next section, this is the best decay rate that can be achieved for generic solutions of NS.

The FS method for the Navier-Stokes was later extended to get the decay of the derivatives $[184,190]$. For the extension of the FS-method the main tool was an energy estimate of the form

$$
\frac{d}{d t} \int_{\mathbb{R}^{n}}\left|D^{m} u\right|^{2} d x \leq-C \int_{\mathbb{R}^{n}}\left|D^{m+1} u\right|^{2}+G(t) d x, \quad x \in \mathbb{R}^{n}, t>0 .
$$

\subsection{Lower bounds}

Throughout this section assume that $f \equiv 0$. As the solutions decay for large time, the influence of the linear terms supersedes the one of the nonlinear ones. To streamline the understanding of the asymptotics, both upper bounds and lower bounds of decay need to be studied. This gives better information on the closeness of the Navier-Stokes and their underlying linear counterpart solutions.

The lower rates of decay were first considered by Schonbek in [182]. In this paper, conditions where introduced to show when solutions to the heat and Navier-Stokes equations have similar lower and upper bounds of rates of decay. The following notation will be used in the sequel, for all $k, l=1, \ldots, n$ :

$$
b_{k, l}=\int_{\mathbb{R}^{n}} y_{l} u_{0, k}(y) \mathrm{d} y, \quad c_{k, l}(u)=c_{k, l}=\int_{0}^{\infty} \int_{\mathbb{R}^{n}} u_{l} u_{k} \mathrm{~d} y \mathrm{~d} s .
$$

The above integrals are convergent provided, e.g., $u_{0} \in L_{\sigma}^{2}\left(\mathbb{R}^{n}\right)$ and $\int_{\mathbb{R}^{n}}(1+|y|)\left|u_{0}(y)\right| d y<$ $\infty$, at least for weak solutions satisfying the energy inequality in its strong form (5). 
Indeed, these assumptions imply that $\int_{\mathbb{R}^{n}} u_{0}(x) d x=0$ and then a simple computation (see [161]) shows that the solution $u_{0}(t)$ of the heat equation in $\mathbb{R}^{n}$ with data $u_{0}$ and zero forcing term satisfies $\left\|u_{0}(t)\right\|_{2} \leq C(1+t)^{-(n+2) / 4}$. Next one has just to apply the time decay estimate on $\|u(t)\|_{2}$ provided by Theorem 2.5 to conclude.

In [187] lower and upper rates of decay are studied for solutions to the MHD system. The results in [187] add a hypothesis on the solutions that was missed in [182] and corrects a gap. The ideas and hypothesis from $[182,187]$ give the basis for the more precise results by Miyakawa-Schonbek in [164].

The main theorem in [164] applies to weak solutions in all space dimensions and states:

Theorem $2.7([164])$. Let $u_{0} \in L_{\sigma}^{2}\left(\mathbb{R}^{n}\right)$, such that $\int_{\mathbb{R}^{n}}(1+|y|)\left|u_{0}(y)\right| d y<\infty$. Let $u$ be a weak solution of $N S$ arising from $u_{0}$ such that $\|u(t)\|_{2}=\mathcal{O}\left(t^{-(n+2) / 4}\right)$ as $t \rightarrow+\infty$ (see Remark 2.6 for the existence of such a solution). The following dichotomy holds:

(i) If $\left\{b_{k, l}\right\}=0$ and if there exists $c \in \mathbb{R}$ such that $\left\{c_{k, l}\right\}=\left\{c \delta_{k, l}\right\}$ (where $\delta_{k, l}=1$ or 0 is the Kronecker symbol) then

$$
\lim _{t \rightarrow \infty} t^{\frac{n+2}{4}}\|u(t)\|_{2}=0
$$

(ii) Otherwise, (i.e., if $\left\{b_{k, l}\right\} \neq 0$ or if $\left\{c_{k, l}\right\}$ is not a scalar multiple of the identity matrix), then there exists $c^{\prime}>0$ such that

$$
\|u(t)\|_{2} \geq c^{\prime} t^{-\frac{n+2}{4}} .
$$

In particular, if $\int x_{k} u_{0, l}(x) d x \neq 0$ for some $k, l \in\{1, \ldots, n\}$, then (11) follows.

Remark 2.8. In view of the fact that the uniqueness of the weak solutions is a major open problem, a priori one cannot exclude the possible situation that for some initial data one could have several weak solutions, possibly with different values of $c_{k, l}$ and with different behavior for $t \rightarrow+\infty$.

Ideas of the proof. Let $u$ be a weak solution of NS starting from $u_{0}$, written in its integral form, as in (2). The integral formulation is indeed valid in the classical setting of weak solution (as well as in more general settings) as thoroughly discussed in [61,143].

Writing this equation component-wise $(j=1, \ldots, n)$ leads to:

$$
u_{j}(t)=\int_{\mathbb{R}^{n}} E_{t}(x-y)\left(u_{0}\right)_{j}(y) \mathrm{d} y-\int_{0}^{t} \int_{\mathbb{R}^{n}} F_{l, j, k}(x-y, t-s) u_{l}(y, s) u_{k}(y, s) \mathrm{d} y \mathrm{~d} s .
$$

Here

$$
E_{t}(x)=(4 \pi t)^{-\frac{n}{2}} e^{-|x|^{2} / 4 t}
$$

is the heat kernel, and $F$ denotes the kernel of the operator $e^{t \Delta} \mathbb{P}$ div. The components of $F$ are given by (see [161])

$$
F_{l, j, k}(x, t)=\partial_{l} E_{t}(x) \delta_{j k}+\int_{t}^{\infty} \partial_{l} \partial_{j} \partial_{k} E_{s}(t) \mathrm{d} y \mathrm{~d} s .
$$


Under the hypothesis of Theorem 2.7, in [73] Fujigaki and Miyakawa established that

$$
\lim _{t \rightarrow \infty} t^{\frac{n+2}{4}}\left\|u_{j}(t)+\partial_{l} E_{t}(\cdot) \int_{\mathbb{R}^{n}} y_{l} u_{0, j}(y) \mathrm{d} y+F_{l, j, k}(x, t) \int_{0}^{t} \int_{\mathbb{R}^{n}}\left(u_{l} u_{k}\right)(y, s) \mathrm{d} y \mathrm{~d} s\right\|_{2}=0
$$

Let $b_{l}=\left(b_{1, l}, \cdots, b_{n l}\right)$ and $F_{l, k}=\left(F_{l, 1, k}, \ldots, F_{l, n, k}\right)$. The key observation in [164] is that $\left\|\partial_{l} E_{t}(\cdot) b_{l}+F_{l k}(\cdot, t) c_{k l}\right\|_{2}=c^{\prime} t^{-(n+2) / 4}$, for some $c^{\prime} \geq 0$ depending on $u$, with $c^{\prime}=0$ if and only if

$$
\begin{aligned}
& \int_{\mathbb{R}^{n}} x_{k} u_{0, l}(x) \mathrm{d} x=0 \quad \forall k, l, \quad \text { and } \\
& \int_{0}^{\infty} \int_{\mathbb{R}^{n}}\left(u_{k} u_{l}\right)(x, t) \mathrm{d} x \mathrm{~d} t=c \delta_{k, l} \quad \text { for some constant } c \geq 0 \text { and } \forall k, l .
\end{aligned}
$$

Applying the trangle inequality to

$$
u(t)=-\left(\partial_{l} E_{t} b_{l}+F_{l, k} c_{k, l}\right)+\left(u(t)+\partial_{l} E_{t} b_{l}+F_{l, k} c_{k, l}\right)
$$

and the limit (14) yields:

$$
\|u(t)\|_{2}=c^{\prime} t^{\frac{n+2}{4}}-o\left(t^{\frac{n+2}{4}}\right) .
$$

This achieves the proof.

In [164], the authors also considered the case of flows arising from data $u_{0} \in L_{\sigma}^{2}$ such that $\left\|e^{t \Delta} u_{0}\right\|_{2} \leq C(1+t)^{-\frac{n}{4}}$ : this is typically the case when $u_{0} \in L^{1} \cap L_{\sigma}^{2}$, even though the integrability is not always necessary. In this case they showed that

$$
\|u(t)\|_{2} \geq c t^{-\frac{n}{4}} \text {, if and only if }\left\|e^{t \Delta} a\right\|_{2} \geq c t^{-\frac{n}{4}} \text { for } t \rightarrow \infty .
$$

The proof follows using triangle inequalities in one direction for $\left\|e^{t \Delta} a\right\|_{2}$ and for $\|u(t)\|_{2}$ in the other, combined with upper bounds of decay for the heat kernel and the estimate (14).

\subsection{Rapidly dissipative solutions and the role of symmetries}

Let $u(x, t)$ be the solution to NS with datum $u_{0}$. By Theorem $2.5,\|u(t)\|_{2}^{2} \leq C(1+t)^{-\alpha}$ with $0<\alpha \leq(n+2) / 2)$, provided such decay holds for the solution $e^{t \Delta} u_{0}$ of the heat equation. As discussed in Section 2.5 the restriction on the exponent $\alpha$ is now known to be generically optimal. See [182] and, more recently, in $[86,87,164]$ with different methods. Accordingly with Wiegner's decay result, any solution of the free Navier-Stokes equation (NS) in $\mathbb{R}^{n}$ will be called rapidly dissipative provided that

$$
\lim _{t \rightarrow+\infty} t^{\frac{n+2}{2}}\|u(t)\|_{2}^{2}=0
$$

Even though Theorem 2.7 provides some sort of necessary and sufficient condition for solutions to be rapidly dissipative, the existence of such solutions (different from the identically zero one) is not an obvious consequence of this theorem. Indeed, the algebraic 
condition $(\mathcal{C})$ on $\int_{0}^{\infty} \int\left(u_{j} u_{k}\right)(y, s) \mathrm{d} y \mathrm{~d} s$ is difficult to check, as it involves the solution itself and not just the initial datum.

However, exceptional flows which decay much faster do exist. For example, it has been known for a long time that, in dimension $n=2$, there exists such an explicit solution of the Navier-Stokes equations with radial vorticity. This condition on the vorticity implies that the velocity field is rotationally invariant and the nonlinearity has potential form (i.e. $\nabla \cdot(u \otimes u)=-\nabla p)$, so that $u$ is also a solution to the homogeneous heat equation. It was pointed out for the first time in [182] (see also [73,164]), that inside this class of flows one can find nonzero solutions with exponential decay at infinity in the $L^{2}$-norm. Specifically, let $\omega_{0}=$ curl $u_{0}$ be a radial function in $\mathbb{R}^{2}$. Let $\omega(t)=\operatorname{curl} u(t)$ and assume that $\nabla u_{0}$ and $\omega_{0}$ satisfy $\omega_{0} \in L^{1}, \nabla u_{0} \in L^{1}, \hat{\omega}(\xi)=0$ for $|\xi| \leq \delta$, some $\delta>0$. An easy computation shows that $u_{0} \in L^{1} \cap L^{2}$, and that $u$ satisfies:

$$
u(x, t)=\frac{1}{r^{2}}\left(\begin{array}{c}
-x_{2} \\
x_{1}
\end{array}\right) \int_{0}^{r} s \omega(s, t) d s,
$$

The velocity remains rotationally invariant for all time and in particular it fulfills condition $(\mathcal{C})$, ensuring that Part (i) of Theorem 2.7 holds. But for this flow, it is established in $[182]$ that

$$
\|u(\cdot, t)\|_{\infty} \leq C \exp \left(-\delta^{2} t\right), \quad \text { and } \quad\|u(\cdot, t)\|_{1} \leq c t .
$$

The exponential decay of the $L^{2}$-norm follows by interpolation.

Similar flows with exponential decay exist in higher even dimension and a general method for their construction is described in [187]. All these solutions, sometimes called generalized Beltrami flows, turn out to solve simultaneously (NS) and the heat equation. As discussed in [187], it seems impossible to adapt these examples to the $n=3$ case or for any other odd dimensions. One difficulty is related to the fact that one is faced with a topological obstruction in attempting to construct nontrivial divergence-free vector fields invariant under rotations. Beside generalized Beltrami flows, a few other exact solutions of the Navier-Stokes equations are known (see e.g. the review paper [202]). However, no examples of rapidly dissipative solution in $\mathbb{R}^{3}$ has been known until Brandolese's construction [18] in 2001 (see also [20]).

The basic idea of [18] is to consider solutions invariant under a suitable discrete subgroup of rotations. For example, a weak solution arising from

$$
u_{0}\left(x_{1}, x_{2}, x_{3}\right)=\left(\begin{array}{c}
x_{1}\left(x_{3}^{2}-x_{2}^{2}\right) e^{-|x|^{2}} \\
x_{2}\left(x_{1}^{2}-x_{3}^{2}\right) e^{-|x|^{2}} \\
x_{3}\left(x_{2}^{2}-x_{1}^{2}\right) e^{-|x|^{2}}
\end{array}\right) .
$$

will inherit the symmetry property of the datum (the parity conditions and the discrete rotational invariance) and the energy decays considerably faster than predicted by Wiegner's theorem (the decay will be $\mathcal{O}\left(t^{-9 / 2}\right)$ as $t \rightarrow+\infty$ for the above example). This behavior is due to the fact that such symmetries force both the velocity and vorticity to have a few additional vanishing moments for all time. The persistence of higher-order vanishing 
moments of the vorticity is also closely related to the persistence during the evolution of orthogonality-type relations, like

$$
\forall t \geq 0, \quad \int\left(u_{j} u_{k}\right)(x, t) \mathrm{d} x=c(t) \delta_{j, k}
$$

which is even stronger than what is actually needed in condition $(\mathcal{C})$. Such orthogonality relations are essential to prevent the spatial spreading effects (the instantaneous loss of localization) on the solution described by Brandolese in [25]. Such type of results can be adapted in the case of flows in the half-space (see [74]) or some other domains with symmetries, see [102].

A complete picture of rapidly dissipative solution with symmetries, in dimension two and three, is presented in [19] by Brandolese, where the best decay rates, depending on the symmetry group, are fully classified.

\subsection{The vorticity approach and more on rapidly dissipative solutions}

The analysis of Th. Gallay and C.E. Wayne [86], [87] (see also Section 2.10.2) provides a deeper insight of rapidly dissipative solutions. Their starting observation is that the decay property (10) is left invariant by time translations of the solution $u$, but the hypothesis $\int(1+|x|)\left|u_{0}(x)\right| \mathrm{d} x<\infty$ needed in Theorem 2.7 to achieve (10) is not. Thus, this hypothesis cannot be optimal.

The work in [87] analyzes the 2-dimensional NS equations. The vorticity is studied after being rescaled and the corresponding velocity is recuperated using the Bio-Savart law. The scaling, already considered in [39], is given by

$$
\xi=\frac{x}{t+1}, \quad \tau=\log (1+t)
$$

yielding the new functions $w(\xi, \tau)$ and $v(\xi, \tau)$ defined by

$$
\omega(x, t)=\frac{1}{t+1} w\left(\frac{x}{t+1}, \log (1+t)\right) \quad u(x, t)=\frac{1}{t+1} v\left(\frac{x}{t+1}, \log (1+t)\right) .
$$

With this scaling the vorticity satisfies the equations in $2 \mathrm{D}$

$$
\partial_{\tau} w=\mathcal{L} w-\left(v \cdot \nabla_{\xi}\right) w
$$

where $\mathcal{L}$ is defined by

$$
\mathcal{L} w=\Delta_{\xi} w+\frac{1}{2}\left(\xi \cdot \nabla_{\xi}\right) w+w
$$

The long time asymptotic is analyzed in this new setting. Linearizing equations (19) around the origin gives a countable set of isolated real eigenvalues corresponding to the time evolution generator: by working with vorticities in the weighted- $L^{2}$ spaces

$$
L^{2}(m)=\left\{f:\|f\|_{L^{2}(m)}^{2} \equiv \int\left(1+|\xi|^{2}\right)^{\frac{m}{2}}|f(\xi)| d \xi<\infty\right\}
$$


with sufficiently large $m$, the essential spectrum can be moved as far as needed to the left half plane. One can then establish the existence of invariant manifolds of finite dimension in the phase space of the vorticity equations. These manifolds are attractors for solutions in neighborhoods of the origin. Ordinary differential equations are obtained by restricting the vorticity partial differential equation to the invariant manifolds. The rate at which the solutions converge to the attracting manifolds is determined by the asymptotics of these ordinary differential equations.

The work in [86] studies the 3 -dimensional NS equations in the spaces $L^{2}(m)$. The solution considered have small initial vorticity which decays algebraically as $|x| \rightarrow \infty$. The same scaling of $2 \mathrm{D}$ is used. The vorticity equations in the new variables in $3 \mathrm{D}$, with $\mathcal{L}$ defined in (20), read as

$$
\partial_{\tau} w=\mathcal{L} w-\left(v \cdot \nabla_{\xi}\right) w+\left(w \cdot \nabla_{\xi}\right) v
$$

The authors in [86] obtain an asymptotic development of the solution as $t \rightarrow+\infty$, in negative powers of $t$. Explicit computations are given for all the first and second order asymptotics. A strong stable manifold of the origin is constructed where the initial vorticity is supposed to be. For solutions with such initial vorticity a new characterization of rapidly dissipative solutions (in the sense of Section 2.6) is obtained. Unlike in Theorem 2.7, no conditions on $u_{0}$ are prescribed that are non-invariant under the flow. On the other hand, a smallness assumption is required on the initial vorticity, but this condition in the end is not really restrictive, as weak solutions constructed so far are eventually small due to to the energy dissipation.

A byproduct of their construction is that there exist nontrivial solutions of the NS equations with energy dissipating at an arbitrary fast algebraic decay rate. With appropriate changes, their method goes through in any space dimension.

\subsection{Asymptotic behavior of global solutions in scale invariant spaces}

Kato's theory described in Section 2.2 provides global solutions $u \in B C\left([0, \infty), L^{n}\left(\mathbb{R}^{n}\right)\right)$, satisfying $\|u(t)\|_{n} \rightarrow 0$, assuming that $\left\|u_{0}\right\|_{n}$ is small enough. In fact, in the decay estimates (1) one could drop the smallness condition on $\left\|u_{0}\right\|_{n}$, replacing it by the assumption that $\|u(t)\|_{n}$ does not blow up: in this case, however, the constants in those estimates would depend on the solution itself. The solutions constructed by T. Kato are in fact the only mild solutions in $B C\left([0, \infty), L^{n}\left(\mathbb{R}^{n}\right)\right)$ with $u(0)=u_{0}$, by G. Furioli, P.-G. LemariéRieusset and E. Terraneo uniqueness theorem, see [143]. On the other hand, in the three dimensional case, there are a few known examples of solutions, with a special geometrical structure, that are global-in-time, do belong to $C\left([0, \infty), L^{3}\left(\mathbb{R}^{3}\right)\right)$ and, arise from "large data" ( this means arbitrarily large in the $L^{3}\left(\mathbb{R}^{3}\right)$-norm, or any other norm of possibly rougher spaces with the same scaling), see the work of Chemin and Gallagher in [49] and the references therein.

A priori, one might think that such solutions do not vanish as $t \rightarrow \infty$. However, I. Gallagher, D. iftimie and F. Planchon [81] proved that all these solutions must decay 
in the $L^{3}\left(\mathbb{R}^{3}\right)$ norm as $t \rightarrow \infty$, no matter what the size of $\left\|u_{0}\right\|_{3}$ is. Their proof uses the structure of the equation and in particular energy estimates on the difference $v=$ $u-w$, where $u$ is the a priori given solution, obtained through the decomposition of $u_{0}$ as $u_{0}=v_{0}+w_{0}$, where $w_{0}$ belongs to $L^{3}\left(\mathbb{R}^{3}\right)$ with small norm (in such a way that one can construct a global solution $w$ from $w_{0}$ via Kato's method) and $v_{0} \in L^{2}\left(\mathbb{R}^{3}\right)$. This idea is due to C. Calderón [31]. Moreover, such global solutions are stable, in the sense that if $\left\|\tilde{u}_{0}-u_{0}\right\|_{3}$ is small enough, then the lifetime of the mild solution $\tilde{u} \in C\left([0, \infty), L^{3}\right)$ arising from $\tilde{u}_{0}$ is infinite and, for all $t \geq 0,\|\tilde{u}(t)-u(t)\|_{3}$ remains bounded by a constant independent on $t$.

These kind of results in fact are not specific to the $L^{3}\left(\mathbb{R}^{3}\right)$ space, similar conclusions hold true e.g. in homogeneous Besov spaces $\dot{B}_{p}^{-1+\frac{3}{p}, q}\left(\mathbb{R}^{3}\right)$, that share with $L^{3}\left(\mathbb{R}^{3}\right)$ the same scaling invariance, see [81]. Results in the same spirit, but with technically different proofs are due to P. Auscher, S. Dubois and Ph. Tchamitchian, see [4]. These authors essentially prove that an a priori global solution $u$ belonging to the Koch-Tataru space [126] must decay to zero in the Koch-Tataru norm as $t \rightarrow \infty$, and are stable in such a space with an analytical dependence on the initial data. As the Koch-Tataru space is the largest scale invariant space where the well-posedness has been established, the results of [4] encompass earlier stability results and are seemingly optimal. The regularity of Koch-Tataru solutions, with decay estimates for higher-order derivatives generalizing estimates (1) has been obtained, e.g. by Germain, Pavlovic and Staffilani [91].

Similar ideas can be used to construct infinite energy global solutions of NS in two dimensions, without putting any smallness assumptions. For example, if $u_{0}$ belongs to the Besov space $\dot{B}_{p}^{-1+\frac{2}{p}, q}\left(\mathbb{R}^{2}\right)$, with $p \geq 2$ and $q>2$, then I. Gallagher and F. Planchon prove that there is a unique solution in $C\left([0, \infty), \dot{B}_{p}^{-1+\frac{2}{p}, q}\right)$. This solution decays to zero in the $\dot{B}_{p}^{-1+\frac{2}{p}, q}\left(\mathbb{R}^{2}\right.$ )-norm (under additional restrictions on $p, q$ ), as shown by P. Germain [90].

\subsection{Decay in weighted spaces and point-wise estimates}

\subsubsection{Early results}

The analysis of the solutions in weighted spaces provides useful information on their spatial behavior. In connection with the energy inequality, it is natural to ask whether weak solutions satisfy the following estimate:

$$
\int|x|^{2 \alpha}|u(x, t)|^{2} \mathrm{~d} x+\int_{0}^{t} \int|x|^{2 \alpha}|\nabla u|^{2}(x, s) \mathrm{d} x \mathrm{~d} s \leq c .
$$

In the case of the Cauchy problem in $\mathbb{R}^{3}$, this estimate was first established with $0 \leq \alpha \leq 3 / 2$ by M.E. Schonbek and T.P. Schonbek [186] for smooth solutions, and then by C. He and Z. Xin [104] for weak solutions emanating from integrable data such that $(1+|x|)^{3 / 2} u_{0} \in L^{2}\left(\mathbb{R}^{3}\right)$. H.-O. Bae and B.J. Jin [7] improved this to $\alpha<\frac{5}{2}$, using CalderónZygmund type inequalities, under slightly more stringent conditions on the decay of the datum. A similar conclusion, valid locally in time and with an additional weighted- $L^{2}$ 
estimate for $\Delta u$ was obtained in [21] by L.Brandolese. The bound $\alpha<\frac{5}{2}$, as pointed out by Brandolese in [25], is generically optimal, as any solutions satisfying estimate (21) with $\alpha=\frac{5}{2}$ must satisfy orthogonality relations of the form (18).

In the setting of strong solutions, there are many results establishing upper bounds for weighted- $L^{p}$ norms of $u$ or its derivatives. This issue is closely related to the problem of establishing point-wise decay estimates. Indeed, from a point-wise decay estimate one immediately obtains weighted estimates by a straightforward integration. Deducing a point-wise estimate for large time from weighted estimates is less obvious, nevertheless, this can be done as will discussed below.

Early results on space-time point-wise decay go back to G.H. Knightly [123], where he addresses such type of decay for solutions to the 3D NS equations with zero forcing term. The initial datum is assumed to be continuous, divergence free and to fulfill the condition

$$
|u(x, 0)| \leq A \min \left\{1,|x|^{-r}\right\}, \quad \text { for some } 1 \leq r<3 .
$$

Provided $A$ is sufficiently small the corresponding solutions are shown to satisfy

$$
|u(x, t)| \leq C \min \left\{1,|x|^{-r}, t^{-\frac{r}{2}}\right\}, \quad x \in \mathbb{R}^{3}, \quad t>0 .
$$

These results were extended by Knightly in $[124,125]$ to any spatial dimension, where in addition he established that the solutions are space-time analytic. The case of nonzero spatial asymptotic behavior was also considered: If $u$ satisfies,

$$
\left|u(x, t)-u_{\infty}\right|=o(1), \quad|\nabla u(x, t)|=o(x), \quad|p(x, t)|=o(x) \text { as }|x| \rightarrow \infty,
$$

where $u_{\infty}$ is a constant, then it is shown that

$$
\begin{gathered}
\left|\nabla^{j} u(x, t)\right| \leq C \min \left\{1,|x|^{-r}, t^{-\frac{r}{2}}\right\} \quad j=0,1, r \in[1, n), \\
|p(x, t)| \leq C t^{-\frac{1}{2}} \min \left\{1,|x|^{-2 r-1}, t^{-r-\frac{1}{2}}\right\}, r \in\left[1, \frac{n}{2}\right) .
\end{gathered}
$$

Note that for the pressure decay the result is only obtained when $n \geq 3$.

A different approach was used in S. Takahashi's paper [199]. Using weighted-equation techniques, Takahashi gets point-wise decay rates both in time and space, under the assumptions that the external force has an algebraic space-time decay rate and the initial datum is zero. The solutions are assumed to be bounded in some weighted $L^{q, s}$ norms, with $\frac{n}{q}+\frac{2}{s}=1$ and $q, s \in[2, \infty]$, (the limiting Serrin class), where $L^{q, s}$ denotes the space of all $u: \mathbb{R}^{n} \times(0, \infty) \rightarrow \mathbb{R}^{n}$ such that

$$
\left(\int_{0}^{\infty}\left(\int_{\mathbb{R}^{n}}|u(x, t)|^{q} \mathrm{~d} x\right)^{\frac{s}{q}} d t\right)^{\frac{1}{s}}<\infty .
$$

In [199] almost optimal uniform space-time decay estimates are established, that is, the estimates are close to the ones corresponding to solutions to the heat equations. For an outline of previous work on space-time decay the reader is referred to [199]. The work of 
C. Amrouche et al. [3] complements and extends the results in [199]. It considers nonzero initial data and zero forcing terms, the space time decay is obtained for the derivatives of all orders in spaces of dimensions $n \leq 5$. The proof combines the decay obtained for moments of the solutions $u$ of NS in $L^{2}$ in [186], the decay of derivatives of the solution $[184,190]$ and a Gagliardo Nirenberg interpolation inequality that yields the decay in $L^{\infty}$ norm of $v(x, t)=\left(1+|x|^{2}\right)^{\frac{k}{2}} D^{\alpha} u$, for $k \leq \frac{n}{2}$. I. Kukavica's paper [136] improves the decay rates computed in [3], obtaining rates similar to those that are available for the heat equations up to the critical rates $|x|^{-n}$ and $t^{-\frac{n}{2}}$. These critical rates were further improved to their optimal values by T. Miyakawa [161], as will be seen in Section 2.9.2.

\subsubsection{Miyakawa's point-wise space-time decay result and its consequences}

T. Miyakawa [161] not only found the optimal point-wise decay rates in space-time, but also got the optimal range of validity for these space-time decay estimates to hold. The slightly improved version below of his theorem (the improvement concerns only the smallness assumption, that in [161] was not scale invariant) is taken from Brandolese [20].

Theorem 2.9 (Miyakawa). Let $1 \leq \gamma \leq n+1$ and let $u_{0}$ be a divergence-free vector field such that

$$
(1+|x|)^{\gamma} u_{0}(x) \in L^{\infty}\left(\mathbb{R}^{n}\right) .
$$

If $\gamma=n, n+1$ it is also assumed that

$$
\sup _{x \in \mathbb{R}^{n}, t \geq 0}(1+|x|)^{\gamma}\left|e^{t \Delta} u_{0}(x)\right|<\infty
$$

and in the case $\gamma=n$, additionally it will be assumed that $t^{\frac{n}{2}}\left|e^{t \Delta} u_{0}(x)\right|$ is uniformly bounded in $x$ and $t$.

If $\sup _{x \in \mathbb{R}^{n}}|x|\left|u_{0}(x)\right|<\eta$, with $\eta>0$ small enough, then there exist a constant $C$ and a solution $u$ of $(N S)$ such that $u(0)=u_{0}$ (e.g. in the distributional sense) and

$$
|u(x, t)| \leq C \eta(1+|x|)^{-\gamma}, \quad|u(x, t)| \leq C \eta(1+t)^{-\frac{\gamma}{2}} .
$$

This theorem can also be seen as a persistence result of the point-wise decay condition $(1+|x|)^{\gamma} u_{0} \in L^{\infty}\left(\mathbb{R}^{n}\right)$. F. Vigneron addressed in [201] the related questions of the persistency (for strong solutions) of a condition like $(1+|x|)^{\gamma} u_{0} \in L^{p}\left(\mathbb{R}^{n}\right)$. In this case, the natural limitation on the exponenent of the weight is $\gamma+\frac{n}{p}<n+1$.

The proof relies in writing the Navier-Stokes equation in its integral form, as in (12):

$$
u(t)=e^{t \Delta} u_{0}-\int_{0}^{\infty} \int F(x-y, t-s)(u \otimes u)(y, s) \mathrm{d} y \mathrm{~d} s
$$

The kernel $F$ is smooth outside the origin and satisfies the scaling invariance $F(x, t)=$ $t^{-\frac{1}{2}(n+1)} F\left(\frac{x}{\sqrt{t}}, 1\right)$ and the bound $|F(x, 1)| \leq C(1+|x|)^{-n-1}$. Combining these two bounds

one sees that $|F(x, t)| \sim|x|^{-\alpha} t^{-\frac{1}{2}(n+1-\alpha)}$ for all $0 \leq \alpha \leq n+1$. Using such information one can perform the relevant estimates on the linear and bilinear terms that eventually 
lead to the desired result by a perturbation argument. Notice that the smallness condition on $u_{0}$ is invariant under the natural scaling of the equation.

The solution of Theorem 2.9 is unique e.g. in $C\left(\left[0, \infty\left[, L_{\gamma}^{\infty}\left(\mathbb{R}^{n}\right)\right)\right.\right.$, where $L_{\gamma}^{\infty}\left(\mathbb{R}^{n}\right)$ is the space of all functions $f$ such that $(1+|x|)^{\gamma} f(x) \in L^{\infty}\left(\mathbb{R}^{n}\right)$ and the continuity in $t=0$ is defined in the distributional sense (as it is usually done in non-separable spaces). The simplest way of ensuring the validity of (24) is to assume $u_{0} \in L^{1}\left(\mathbb{R}^{n}\right)$ (if $\gamma=n$ ) or $(1+|x|) u_{0} \in L^{1}\left(\mathbb{R}^{n}\right)$ (if $\gamma=n+1$ ). However, these latter conditions instantaneously break down (unless there are symmetries) whereas condition (24) remains true during the evolution.

For simplicity, focus is now centered on the case of fast decaying initial data, i.e. such that $(1+|x|)^{\gamma} u_{0} \in L^{\infty}$ with $\gamma \geq n+1$. From the point-wise estimates (25) one readily deduces decay estimates in weak-Lebesgue norm $L_{w}^{q}$ of $u$. Next interpolating the $L^{p}$ norm between two different weak norms, $L_{w}^{q}$ and $L_{w}^{q^{\prime}}, q<p<q^{\prime}$, leads to (the $p=1$ case would require in fact the use of weak-Hardy spaces, see [160]):

$$
\left\|u(\cdot, t)(1+|x|)^{\alpha}\right\|_{p}=\mathcal{O}\left(t^{-\frac{1}{2}\left(n+1-\alpha-\frac{n}{p}\right)}\right),
$$

valid for all $1 \leq p \leq \infty$ and $\alpha \geq 0$ such that $\alpha+\frac{n}{p}<n+1$, (or $0 \leq \alpha \leq n+1$ if $p=\infty)$. For more details on this argument, see the work by Bae and Brandolese [5]. For the corresponding lower bounds of these weighted norms, see Section 2.10.1.

The arguments in [161] could be used to obtain estimates for the derivatives of any order: in agreement with what happens for the heat equation, an estimate for a spatial derivative of order $b \in \mathbb{N}^{n}$ improves the above decay rate of $u$ of an exponent $-|b| / 2$.

I. Kukavica ans J.J. Torres $[137,140]$ proposed a different approach to such kind of estimates. Namely, using a parabolic interpolation inequality they proved that if $u_{0} \in$ $\mathcal{S}\left(\mathbb{R}^{n}\right)$ is an initial datum leading to a strong solution such that $\|u(t)\|_{2}=\mathcal{O}\left(t^{-\mu}\right)$, for some $\mu \geq 0$, then (provided $0 \leq \alpha<n+1-d / p$ ),

$$
\left\|D^{b} u(\cdot, t)(1+|x|)^{\alpha}\right\|_{p}=\mathcal{O}\left(t^{-\frac{1}{2}\left(\frac{n}{2}+2 \mu+b-\alpha-\frac{n}{p}\right)}\right) .
$$

Notice that the assumption $u_{0} \in \mathcal{S}\left(\mathbb{R}^{n}\right)$ is enough to guarantee that one can take $\mu \geq$ $(n+2) / 4$, by Wiegner's theorem (Theorem 2.5). Hence for such data:

$$
\left\|D^{b} u(\cdot, t)(1+|x|)^{\alpha}\right\|_{p}=\mathcal{O}\left(t^{-\frac{1}{2}\left(n+1+|b|-\alpha-\frac{n}{p}\right)}\right),
$$

for all $0 \leq \alpha<n+1-\frac{n}{p}, b \in \mathbb{N}^{n}$, and $1 \leq p \leq \infty$.

When there is no weight $(\alpha=0)$, a different approach leading to bounds of the form of (28) consists in deriving Gevrey-type estimates for the solutions, as done by M. Oliver and E.S. Titi, [172]. The key point is to derive a differential inequality for the norms $G_{r}=\left\|A^{r} e^{\tau(t)} A\right\|_{2}^{2}$, where $A=\sqrt{-\Delta}$ and appropriate choices of $\tau=\tau(t)$ that can be used to obtain explicit bounds on the radius of analyticity of the solution in time. An interesting feature of this approach is that it provides also some lower bounds for the $L^{2}$-large time behavior of the solutions and their derivatives. 


\section{$2.10 \quad$ Asymptotic profiles}

\subsubsection{Asymptotic profiles: the case of well localized velocities}

A. Carpio [41] proved that strong solutions to NS admit an asymptotic expansion in terms of space-time derivatives of the heat kernel. Her results were improved by Y. Fujigaki and T. Miyakawa [72]. To present their asymptotic profile, some notation is needed: recall that $F$ denotes the kernel of the operator $e^{t \Delta} \mathbb{P}$ div appearing in the integral formulation (IE) of the Navier-Stokes equation. More explicitly, $F(x, t)=\left(F_{l, j, k}(x, t)\right)$, and the components of $F$ are given by expression (13).

Denote, as before, the heat kernel by $E(x, t)$ and

$$
\mathcal{E}_{h, k}(t)=\int_{\mathbb{R}^{d}}\left(u_{h} u_{k}\right)(y, t) \mathrm{d} y
$$

the so-called energy matrix of the flow. Notice that $\int_{0}^{\infty} \mathcal{E}_{l, k}(s) \mathrm{d} s=c_{l, k}$ according to the notations introduced just before Theorem 2.7.

Theorem 2.10 ([72]). Let $u_{0}$ be a divergence-free vector field such that $(1+|x|) u_{0} \in$ $L^{1}\left(\mathbb{R}^{n}\right)$ and $u_{0} \in L^{\infty}\left(\mathbb{R}^{n}\right)$, giving rise to a unique global strong solution $u$ (this is always the case if $n=2$, or, e.g., $n \geq 3$ and $\left\|u_{0}\right\|_{n}$ is small enough). Then, for any $q \in[1, \infty]$ and $j=1, \ldots, n$, one has

$$
\lim _{t \rightarrow \infty} t^{\frac{1}{2}+\frac{n}{2}\left(1-\frac{1}{q}\right)}\left\|u_{j}(t)+\partial_{k} E(t, \cdot) \int y_{k} u_{0, j}(y) d y+F_{l, j, k}(t, \cdot) \int_{0}^{\infty} \mathcal{E}_{l, k}(s) d s\right\|_{q}=0
$$

where the summation symbols over repeated subscripts has been omitted.

The above limit does indeed describe the large time behavior of $u$, since by scaling reasons each one of the terms $\partial_{k} E(\cdot, t)$ and $F_{l, j, k}(x, \cdot)$ decay exactly as $t^{\frac{1}{2}+\frac{n}{2}\left(1-\frac{1}{q}\right)}$. The expansion (30) corresponds to a first-order asymptotic profile. Putting more stringent assumptions on the localization of $u_{0}$ (namely under the assumptions of Theorem 2.9 with $\gamma=n+1)$ then one can write a higher order asymptotics for $u$, up to the order $n$ that read as follows:

$$
\begin{aligned}
u_{j}(\cdot, t)= & \sum_{1|\leq| \alpha \mid \leq m} \frac{(-1)^{|\alpha|}}{\alpha !}\left(\partial_{x}^{\alpha} E(x, \cdot) \int y^{\alpha} u_{0, j} \mathrm{~d} y\right. \\
& +\sum_{|\beta|+2 p \leq m-1} \frac{(-1)^{|\beta|+p}}{p ! \beta !}\left(\partial_{t}^{p} \partial_{x}^{\beta}\right) F_{l, j, k} \int_{0}^{\infty} s^{p} y^{\beta}\left(u_{l} u_{k}\right)(y, s) \mathrm{d} y \mathrm{~d} s+\text { l.o.t. },
\end{aligned}
$$

where the neglected terms are of lower-order in the $L^{q}$-norm $(1 \leq q \leq \infty)$, as $t \rightarrow \infty$.

Theorem 2.10 has several variants. One of these applies to weak solutions (in this case one only assumes $u \in L_{\sigma}^{2}\left(\mathbb{R}^{n}\right)$ and $(1+|x|) u_{0} \in L^{2}\left(\mathbb{R}^{n}\right)$ where expansion (30) holds for $1 \leq q \leq 2$. The special case $q=2$ found an important application in Theorem 2.7 as already discussed. H.Y. Choe and B.J. Jin [51] extended (30) by allowing weight terms in the expansion. 
One drawback of the asymptotic expansions like the above or that in Eq. (30) is that any point-wise information on the velocity field is lost when computing the $L^{q}$-norm. However, L. Brandolese and F. Vigneron [27] noticed that for mild decaying solutions (namely, the solutions obtained in Theorem 2.9 with $\gamma>(n+1) / 2$ ), the following pointwise asymptotic profile holds as $t \rightarrow \infty$ :

$$
u(x, t)=e^{t \Delta} u_{0}(x)+\nabla h(x)+r(x, t)
$$

Here $\nabla h(x)$ is a time-independent homogeneous gradient field of degree $-(n+1)$, smooth for $x \neq 0$. The remainder term $r(x, t)$ is a lower-order term as $x$ goes to infinity: actually it satisfies

$$
\lim _{t, \frac{|x|}{\sqrt{t}} \rightarrow \infty}|x|^{n+1} r(x, t)=0 .
$$

In fact, the second term in the right-hand side of (31) is almost explicit (it depends, however, on the solution), as

$$
h(x)=c_{n} \sum_{h, k}\left(\frac{\delta_{h, k}}{n|x|^{n}}-\frac{x_{h} x_{k}}{|x|^{n+2}}\right) \cdot \int_{0}^{\infty} \mathcal{E}_{h, k}(s) \mathrm{d} s .
$$

The homogeneous function $h(x)$ is closely related to the kernel $\mathbb{K}$ of $e^{t \Delta} \mathbb{P}$. Indeed, $\mathbb{K}(x, t)=h(x)+|x|^{-d} \Psi(x / \sqrt{t})$, where $\Psi$ is a Gaussian-like decaying function. Profile (31) follows taking both $t \rightarrow \infty$ and $|x| / \sqrt{t} \rightarrow \infty$ in a more general formula in Brandolese and Vigneron [27, Theorem 1.7], that will not be reproduced here.

Expansion (31) and its variants have several consequences. As noticed in [27], it can be used to derive the corresponding lower bounds for the weighted estimates (27), generalizing those of Section 2.5. Indeed, integrating (31) in portions of the conic region $|x| \geq A \sqrt{t}$, with $A \gg 1$ one easily deduces

$$
\left\|(1+|x|)^{\alpha} u(t)\right\|_{p} \geq \kappa t^{-\frac{1}{2}\left(n+1-\alpha-\frac{n}{p}\right)},
$$

for all $1 \leq p<\infty$ and $\alpha \geq 0$ such that $\alpha+\frac{n}{p}<d+1$ (or $\alpha \leq n+1$ when $p=\infty$ ). Inequality (32) extends earlier lower bounds established in particular cases (namely, for $p=2$ and $0 \leq \alpha \leq 2$, see $[7,86,182]$, or $1 \leq p \leq \infty$ and $\alpha=0$, see [73]). The above constant $\kappa \geq 0$ is strictly positive as soon as $\nabla h(x) \not \equiv 0$, and this is what happens generically. In fact, accordingly with [182] and [164] (see also Theorem 2.7), $\nabla h(x) \equiv 0$ if and only if the matrix $\left(c_{l, k}\right)=\left(\int_{0}^{\infty} \mathcal{E}_{l, k}(s) \mathrm{d} s\right)$ is a scalar multiple of the identity. Of course, for symmetric solutions $\nabla h(x)$ can be identically zero.

An expansion similar to (31) is also meaningful for fixed $t$ and $|x| \rightarrow \infty$. In this case the result of [27] asserts that, for mild decaying solutions (possibly defined only locally in time)

$$
u(x, t)=e^{t \Delta} u_{0}(x)+H(x): K(t)+o_{t}\left(|x|^{-n-1}\right), \quad \text { as }|x| \rightarrow+\infty,
$$

where $H(x)=c_{n} \nabla\left(\frac{\delta_{h, k}}{n|x|^{n}}-\frac{x_{h} x_{k}}{|x|^{n+2}}\right)$, is homogeneous of degree $-(d+1), c_{n}$ is an absolute constant, $K(t)=\int_{0}^{t} \mathcal{E}_{h, k}(s) \mathrm{d} s$, the symbol “:" stands for a double summation 
on $h, k=1, \ldots, n$ and the $o_{t}\left(|x|^{-n-1}\right)$ notation denotes a function $\mathcal{R}(x, t)$ such that $\lim _{x \rightarrow \infty}|x|^{n+1} \mathcal{R}(x, t)=0$ for all fixed $t$. Thus, if $u_{0}$ is well localized (say $u_{0} \in \mathcal{S}\left(\mathbb{R}^{n}\right)$ ), then

$$
u(x, t) \sim H(x): K(t), \quad \text { as } x \rightarrow \infty
$$

for all fixed $t$ such that the strong solution is defined. This expansion can be used to establish point-wise lower bounds as $|x| \rightarrow \infty$. I. Kukavica and E. Reis [138] proposed a variant to (31), involving a higher-order expansion.

\subsubsection{Asymptotic profiles: the case of poorly localized velocities}

M. Cannone, C. He and G. Karch [34] established an analogue of the expansion (30) for strong solutions with slower decay, arising from inital data small in $L^{n}\left(\mathbb{R}^{n}\right)$ and belonging to the weak Lebesgue space $L_{w}^{\frac{n}{\alpha}}\left(\mathbb{R}^{n}\right)$, for some $\alpha \in[1, n)$. Let us recall for $1<p<\infty$ the space $L_{w}^{p}=L^{p, \infty}$ is normed by

$$
\|v\|_{p, \infty}=\sup _{r>0} r\left|\left\{x \in \mathbb{R}^{n}:|v(x)|>r\right\}\right|^{\frac{1}{p}}
$$

The results in [34] yield the second terms of the asymptotic expansions of the solutions to NS in $\mathbb{R}^{n}$. Their result applies to the class of global solutions satisfying the following estimates, for $1<\alpha<n$ :

$$
\sup _{t>0}\|u(t)\|_{n / \alpha, \infty}<\infty,\|u(t)\|_{p}<C(1+t)^{-\frac{n}{2}\left(\frac{\alpha}{n}-\frac{1}{p}\right)},\|\nabla u(t)\|_{p} \leq C t^{-\frac{1}{2}}(1+t)^{-\frac{n}{2}\left(\frac{\alpha}{n}-\frac{1}{p}\right)} .
$$

One of the main results in [34] is

Theorem 2.11 ([34]). Let $u$ be a solution of $N S$ satisfying (34). If $1<\alpha<1+\frac{n}{2}$, then for any $p \in[1, \infty]$ satisfying $p>n /(2 \alpha)$

$$
\lim _{t \rightarrow \infty} t^{\frac{n}{2}\left(\frac{\alpha}{n}-\frac{1}{p}\right)+\frac{n-1}{2}}\left\|u(t)-e^{t \Delta} u_{0}+\int_{0}^{t} \int_{\mathbb{R}^{n}} e^{(t-\tau) \Delta} \mathbb{P} \nabla \cdot(\tilde{u} \otimes \tilde{u})(y, \tau) d y d \tau\right\|_{p}=0
$$

for all $t>0$, where $\tilde{u}=e^{\tau \Delta} u_{0}=E(\cdot, \tau) * u_{0}$.

Remark 2.12. Estimates (34) hold for the unique mild solution $u_{0}$ arising e.g. from small data in $u_{0} \in L^{n / \alpha, \infty} \cap L^{\infty}\left(\mathbb{R}^{n}\right)$.

The authors of [34] complete their theorem studying the case $\alpha>1+\frac{n}{2}$ : in this case they obtain essentially the same profile as in Theorem 2.10. The proof follows by writing the solution in the appropriate integral form and using an adaptation of the method in [73] by Fujigaki and Miyakawa. 


\subsubsection{Asymptotic profiles: the self-similar case}

The two dimensional case is considered first. When the initial vorticity $\omega_{0}=\nabla \times u_{0}$ is in $L^{1}\left(\mathbb{R}^{2}\right.$ ), by the classical results of M. Ben Artzi and T. Kato (see e.g. [9]), there is a unique global solution $\omega \in C\left([0, \infty), L^{1}\left(\mathbb{R}^{2}\right)\right) \cap C\left((0, \infty), L^{\infty}\left(\mathbb{R}^{2}\right)\right)$ of the vorticity equation

$$
\partial_{t} \omega+(u \cdot \nabla) \omega=\Delta \omega, \quad x \in \mathbb{R}^{2}, t>0
$$

arising from $\omega_{0}$. Such solution is also known to decay as $\|\omega(t)\|_{p}=\mathcal{O}\left(t^{1-\frac{1}{p}}\right)$ as $t \rightarrow \infty$, for $1 \leq p \leq \infty$.

The simplest explicit self-similar solutions of the Navier-Stokes equation, in 2D, are the Oseen vortices,

$$
\omega_{\text {Oseen }}(x, t)=\frac{\alpha}{4 \pi t} e^{-\frac{|x|^{2}}{4 t}}, \quad u_{\text {Oseen }}(x, t)=\frac{\alpha}{2 \pi} \frac{x^{\perp}}{|x|^{2}}\left(1-e^{-\frac{|x|^{2}}{4 t}}\right), \quad x \in \mathbb{R}^{2}, \quad t>0 .
$$

The constant parameter $\alpha \in \mathbb{R}$ is the total circulation of the vortex, $\alpha=\int \omega(x, t) \mathrm{d} x$. Such solutions play a key role in the description of the large time behavior of solutions to the vorticity equation with infinite energy, as illustrated by Theorem 2.13 below. This theorem was first established, by Y. Giga and Kambe [94], under the additional assumption that $\left\|\omega_{0}\right\|_{1}$ is small. Such smallness assumption was subsequently relaxed by A. Carpio in [40] and finally completely removed by Th. Gallay and C.E. Wayne in [88]. See also [93, Chapter 2].

Theorem 2.13 ([88]). Let $\omega \in L^{1}\left(\mathbb{R}^{2}\right)$. Then the solution $\omega$ to (35) behaves as $t \rightarrow \infty$ like the Oseen vortex with circulation $\alpha=\int \omega_{0}$ :

$$
\lim _{t \rightarrow \infty} t^{1-\frac{1}{p}}\left\|\omega(\cdot, t)-\omega_{\text {Oseen }}(\cdot, t)\right\|_{p}=0 \quad(1 \leq p \leq \infty),
$$

and

$$
\lim _{t \rightarrow \infty} t^{\frac{1}{2}-\frac{1}{q}}\left\|u(\cdot, t)-u_{\text {Oseen }}(\cdot, t)\right\|_{q}=0 \quad(2<q \leq \infty) .
$$

See also [87] for higher order asymptotics. The main idea in [88] is the discovery of a new Lyapunov function: the relative entropy of the distribution of the vorticity with respect to the Gaussian distribution of the Oseen kernel. When $p=1$, the above result provides no convergence rate to the Oseen vortex. However, at least when $\omega_{0}$ is very well localized (e.g. when it belongs to a $L^{1}\left(\mathbb{R}^{2}\right)$ space with a Gaussian weight), Th. Gallay and L.M. Rodrigues did establish that such convergence holds at a logaritmic rate. See [85].

A consequence of Theorem 2.13 is that the Oseen vortices are the only self-similar and integrable solutions of the vorticity equation in 2D. However, plenty of self-similar solutions exist such that $\omega(\cdot, t) \notin L^{1}\left(\mathbb{R}^{n}\right)$. As a matter of fact, Y. Giga and T. Miyakawa [96] proposed a general method, based on the analysis of the vorticity equation in Morrey spaces, for constructing non-stationary self-similar solutions to NS in $\mathbb{R}^{3}$, and for two dimensions in [96] it is stated that: 
When the space dimension is two and the total variation of the initial measure is small, there is only one forward self-similar solution for a given total circulation [97]; moreover according to [94] the forward self-similar solutions describe large time behavior of the vorticity. Existence of many self-similar solutions therefore suggests that the large time behavior of the vorticity is much more complicated in the three-dimensional case compared with the two-dimensional case.

More direct constructions have been proposed by Cannone, Meyer, Planchon [37,38], see also [143, Chapt. 23]. Their constructions go through in any spatial dimension $n \geq 2$ : basically, one only has to choose a vector field $u_{0}(x)$ in $\mathbb{R}^{n}$, homogeneous of degree -1 , satisfying some mild smallness and regularity assumption on the sphere $\mathbb{S}^{n-1}$ to guarantee the existence and uniqueness of a self-similar solution emanating from $u_{0}$.

In [176], F. Planchon provided a condition implying that a solution $u(x, t)$ of the Navier-Stokes equations in $\mathbb{R}^{n}$ has a self-similar behavior for large time. Roughly, his results asserts that if $u_{0}$ is small enough in the Besov spaces $\dot{B}_{p}^{-1+\frac{n}{p}, \infty}\left(\mathbb{R}^{n}\right)$ (for some $p>n)$, and is such that $\sqrt{t} e^{t \Delta} u_{0}(\sqrt{t} \cdot) \rightarrow v$ in $L^{p}\left(\mathbb{R}^{n}\right)$ as $t \rightarrow \infty$, then the weak limit $v_{0}(x)=\lim _{\lambda \rightarrow \infty} \lambda u_{0}(\lambda x)$ does exist, next $v=e^{\Delta} v_{0}$, and $u(\cdot, t) \simeq \frac{1}{\sqrt{t}} V\left(\frac{\dot{1}}{\sqrt{t}}\right)$ as $t \rightarrow \infty$, where $\frac{1}{\sqrt{t}} V\left(\frac{x}{\sqrt{t}}\right)$ is the self-similar solution of (NS) arising from $v_{0}$. As observed in [176], it would not be enough to assume that the rescaled data $\lambda u_{0}(\lambda x)$ converge to get the same conclusion.

A byproduct of Planchon's results is that if $u_{0}(x) \sim|x|^{-1} \zeta(x /|x|)$ as $x \rightarrow \infty$, where $\zeta$ is in $L^{\infty}$ on the unit sphere with small enough $L^{\infty}$-norm, then the corresponding solution is asymptotically self-similar. If it is only known that $\left|u_{0}(x)\right| \leq \epsilon|x|^{-1}$ (with $\epsilon>0$ small enough) a quite different situation occurs. In this case, it is still possible to construct a unique global solution $u(x, t)$ such that $|u(x, t)| \leq C \epsilon(|x|+\sqrt{t})^{-1}$. However, Th. Cazenave, F. Dickstein and F. Weissler, in [45], showed that the large time behavior for such solution can be chaotic: They proved that if a sequence of dilates $\lambda_{n} u_{0}\left(\lambda_{n} \cdot\right)$ converges weakly to a solenoidal vector field $z(\cdot)$, when $\lambda_{n} \rightarrow \infty$, then, for $t_{n}=\lambda_{n}^{2}$, the discretely rescaled solution $\sqrt{t_{n}} u\left(\cdot \sqrt{t_{n}}, t_{n}\right)$ will converge as $n \rightarrow \infty$ toward a limit depending on $z$ (namely, $\mathcal{S}(1) z$, where $\mathcal{S}(t)$ is the Navier-Stokes flow). Now, if $t_{n}^{\prime}=\left(\lambda_{n}^{\prime}\right)^{2} \rightarrow \infty$ is another time sequence, it can happen that $\lambda_{n}^{\prime} u_{0}\left(\lambda_{n}^{\prime} \cdot\right)$ converges to a vector field $z^{\prime}(\cdot)$ different from $z(\cdot)$. In this case, the solution $u$ has at least two different asymptotic behaviors along different sequences. In [45], the authors succeeded in constructing initial data $u_{0}$ leading to solutions of the Navier-Stokes equation with infinitely many different asymptotic behaviors, along different time sequences.

\subsection{Decay in Besov spaces and frequency concentration effects}

\subsubsection{Miyakawa's $X^{q}$ spaces}

The asymptotic profile in Theorem 2.10 describes the long time behavior of $u$ in the $L^{q}$-norm, $1 \leq q \leq \infty$, In [163], T. Miyakawa addressed the problem of establishing the analogue result for $q<1$. As the $L^{q}$ spaces are no longer suitable for this purpose, he 
introduced the spaces $X^{q}$ :

$$
X^{q}= \begin{cases}L^{q}, & 1 \leq q \leq \infty, \\ \dot{B}_{1,1}^{n\left(1-\frac{1}{q}\right)}, & 0<q<1, q \neq \frac{n}{n+m}, m \in \mathbb{N} \\ \dot{B}_{1, \infty}^{-m}, & q=\frac{n}{n+m}, m \in \mathbb{N}\end{cases}
$$

The main result in [163] reads as follow

Theorem $2.14([163])$. Let $u_{0} \in L_{\sigma}^{n}\left(\mathbb{R}^{n}\right), \int_{\mathbb{R}^{n}}(1+|y|)\left|u_{0}(y)\right| d y<\infty$ and suppose $\left\|u_{0}\right\|_{n}$ small if $n \geq 3$. Then the strong solution with datum $u_{0}$ satisfies (30), with the norm $\|\cdot\|_{X^{q}}$ replacing the $L^{q}$ norm, for all $\frac{n}{n+1} \leq q \leq \infty$.

If instead $u_{0} \in L_{\sigma}^{2}\left(\mathbb{R}^{n}\right)$, and $\int_{\mathbb{R}^{n}}(1+|y|)\left|u_{0}(y)\right| d y<\infty$, then there is a weak solution satisfying the same profile in the $X^{q}$ norm for $\frac{n}{n+1} \leq q \leq 2$. In particular, the two-sided bound holds for large $t$,

$$
0<c_{q}^{\prime} \leq t^{\frac{n}{2}\left(1+\frac{1}{n}-\frac{1}{q}\right)}\|u(t)\|_{X^{q}} \leq c_{q}
$$

for appropriate constants $c_{q}^{\prime}, c_{q}$ if and only if $u_{0}$ and $u$ do not satisfy condition $(\mathcal{C})$ of Section 2.6 (i.e., if and only if the moments $\int x_{k} u_{0}(x) d x$ are not all zero, or the matrix $\int_{0}^{\infty} \int\left(u_{k} u_{l}\right)$ is not a sclar multiple of the identity matrix). As before, the conditions on $q$ are $\frac{n}{n+1} \leq q \leq \infty$ if $u$ is a strong solution, and $\frac{n}{n+1} \leq q \leq 2$ if $u$ is a weak solution.

To establish the above theorem, the solutions are expressed in integral form. Estimates similar to (14) and from Theorem 2.7 are obtained, however the analysis in Besov spaces is more involved and technical.

\subsubsection{Further applications of Besov spaces and Z. Skalák analysis on fre- quency concentration}

Other decay results in Besov's spaces can be found in Z. Skalák's paper [195]. Skalák considered data $u_{0} \in L_{\sigma}^{2} \cap \dot{B}_{2}^{-2 \gamma, \infty}\left(\mathbb{R}^{3}\right), \gamma \in\left[0, \frac{5}{4}\right]$, or equivalently, data $u_{0}$ such that $\left\|e^{t \Delta} u_{0}\right\|_{2} \leq C(1+t)^{-\gamma}$, by the well known characterization of negative Besov spaces in terms of heat kernel decay (see [143]). Combining the results in [195] the main result can be given as

Theorem 2.15 ([195]). Let $u_{0} \in L_{\sigma}^{2} \cap \dot{B}_{2}^{-2 \gamma, \infty}\left(\mathbb{R}^{3}\right), \gamma \in\left[0, \frac{5}{4}\right]$. Let $u$ be a weak solution to $N S$, with data $u_{0}$. Let $\kappa \in(0,1)$. Then there exists $\beta, \tilde{\beta}>0$, such that

$$
\lim \inf _{t \rightarrow \infty} \frac{\left\|\left(E_{\mu(t)}-E_{\tilde{\mu}(t)}\right) u(t)\right\|_{2}}{\|u(t)\|_{2}} \geq \kappa, \text { where } \mu(t)=\beta t^{-1} \text {, and } \tilde{\mu}(t)=\tilde{\beta} t^{-1} .
$$

Further if $\alpha \geq 0$, then

$$
\liminf _{t \rightarrow \infty} t^{\frac{\alpha}{2}}\|u(t)\|_{\dot{B}_{2}^{-2 \gamma+\alpha, \infty}}>0
$$

Here $\left\{E_{\lambda}: \lambda \geq 0\right\}$ denotes the resolution of the identity of the Stokes operator. The proof of Theorem 2.15 is done in several short lemmas and propositions that use properties of Besov spaces and the spectral family $E_{\mu(t)}$. 
The decay result of Thorem 2.15 is first shown for weak solutions that satisfy :

$$
0<\liminf _{t \rightarrow \infty} t^{\gamma}\|u(t)\|_{2} \leq \limsup _{t \rightarrow \infty} t^{\gamma}\|u(t)\|_{2}<\infty .
$$

The second theorem of [195] shows that condition (38) is equivalent with $u_{0} \in \dot{B}_{2, \infty}^{-2 \gamma}$. For solutions with datum as in Theorem above or satisfying (38), the work in [195] improves the results in [163] and of [164] as follows:

1. A lower bound of decay is obtained for any $\gamma \in\left(0, \frac{5}{4}\right]$.

2. If $\gamma=5 / 4$ the condition from [164]: $\int(1+|x|)\left|u_{0}(x)\right| d x<\infty$ is no longer required.

3. By the continuos imbedding $\dot{B}_{1, \infty}^{-1} \hookrightarrow \dot{B}_{2, \infty}^{-\frac{5}{2}}$ the lower bound obtained in Theorem 2.15

$$
0<\lim \inf _{t \rightarrow \infty}\|u(t)\|_{\dot{B}_{2, \infty}^{-\frac{5}{2}}}
$$

improves the lower bound in [163] given by $0<\liminf \operatorname{in}_{t \rightarrow \infty}\|u(t)\|_{\dot{B}_{1, \infty}^{-1}}$.

Notice that (37) provides information on the long time behavior of frequencies. In a series of papers (see [194] and the references therein) Z. Skalák gave new insight on the large time behavior of the solutions. He showed that a certain frequency concentration effect occurs for weak solutions with zero external forces, satisfying the strong energy inequality (5). We first recall the notation given in [195], let $a=\lim _{t \rightarrow+\infty} \frac{\left\|A^{1 / 2} u\right\|_{2}}{\|u\|_{2}}$, and define

$$
K_{a, \epsilon}= \begin{cases}B_{\sqrt{a+\epsilon}} \backslash B_{\sqrt{a-\epsilon}} & \text { if } a>\epsilon>0, \\ B_{\sqrt{a+\epsilon}}(0) & \text { if } \epsilon \geq a>0, \\ B_{\sqrt{\epsilon}}(0) & \text { if } a=0\end{cases}
$$

where $B_{r}(0)$ denotes a ball centered at the origin with radius $r$.

In [194] it is shown that frequencies tend to concentrate on an arbitrary small annulus $(a>0)$ or ball $(a=0)$ as $t \rightarrow \infty$. In particular it is established that for such weak solutions, which are non zero for times lager than some $T \geq 0$

$$
\lim _{t \rightarrow+\infty} \frac{\int_{\mathbb{R}^{3} \backslash K_{a, \epsilon}}|\xi|^{4 \alpha}|\widehat{u}(\xi, t)|^{2} \mathrm{~d} \xi}{\int_{K_{a, \epsilon}}|\xi|^{4 \alpha}|\widehat{u}(\xi, t)|^{2} \mathrm{~d} \xi}=0 .
$$

\subsection{Decay characterization to dissipative equations}

It has been frequently remarked that the long time behavior in $L^{2}$ of solutions and derivatives to diffusive systems, is influenced by the low frequencies of the initial data. This suggested the idea of finding the order of $\widehat{\Lambda}^{s} u_{0}(\xi)$ at the origin, by comparing it to the powers $f(\xi)=|\xi|^{r},[13]$, where as usual, $\Lambda=(-\Delta)^{\frac{1}{2}}$. For this comparison the notions of decay character and decay indicator were introduced by Bjorland and Schonbek in [13] and its use and applications extended by Niche and Schonbek in [169], [170]. The decay character and the Fourier Splitting method yielded upper and lower bounds for decay of solutions and derivatives to appropriate dissipative nonlinear equations, incompressible and compressible. We recall the definitions of decay indicator and decay character. For details see [13] and [169] 
Definition 2.16. ([13,169]) Let $u_{0} \in L^{2}\left(\mathbb{R}^{n}\right), B(\rho)=\{\xi:|\xi| \leq \rho\}, s \geq 0$. The decay indicator $P_{r}^{s}\left(u_{0}\right), r \in\left(-\frac{n}{2}+s, \infty\right)$ corresponding to $\Lambda^{s} u_{0}$ is defined by

$$
P_{r}^{s}\left(u_{0}\right)=\lim _{\rho \rightarrow 0} \rho^{-2 r-n} \int_{B(\rho)}|\xi|^{2 s}\left|\widehat{u}_{0}(\xi)\right|^{2} d \xi
$$

provided the limit exists.

Definition 2.17. ([169]) For $u_{0} \in L^{2}\left(\mathbb{R}^{n}\right)$, the decay character of $\Lambda^{s} u_{0}$, is defined by

$$
r_{s}^{*}=r_{s}^{*}\left(u_{0}\right)= \begin{cases}\text { the unique } r \in\left(-\frac{n}{2}+s, \infty\right), & \text { if } 0<P_{r}^{s}\left(u_{0}\right)<\infty, \\ -\frac{n}{2}+s, & \text { if } P_{r}^{s}\left(u_{0}\right)=\infty, \forall r \in\left(-\frac{n}{2}+s, \infty\right), \\ \infty, & \text { if } P_{r}^{s}\left(u_{0}\right)=0, \forall r \in\left(-\frac{n}{2}+s, \infty\right) .\end{cases}
$$

The decay character could not exist, for example when $\widehat{u}_{0}(\xi)$ is wildly oscillating near $\xi=0$, or when $\widehat{u}_{0}(\xi)$ has a logarithmic behavior as $\xi \rightarrow 0$. For this reason, improved definitions of the notions of decay indicator and decay character have been recently proposed by L. Brandolese [24]. The class of initial data admitting a decay character in the sense of [24] is larger, making the theory more widely applicable. The modified definitions are not reproduced here. The two next subsections provide a few interesting applications of these notions.

\subsubsection{Characterization of decay in $L^{2}$ and in $\dot{H}^{s}$ of solutions to linear dissi- pative equations}

Let $\mathcal{L}: X^{n} \rightarrow\left(L^{2}\left(\mathbb{R}^{n}\right)\right)^{n}$, be a a pseudo-differential operator on an $n$-dimensional Hilbert space $X^{n}$, with symbol $\mathcal{M}(\xi)=P^{-1}(\xi) D(\xi) P(\xi)$, $\xi$ a.e., where $D(\xi)=-c_{i}|\xi|^{2 \alpha} \delta_{i j}$, for $c_{i}>c>0$ and $0<\alpha \leq 1$ and $P(\xi) \in O(n)$. Let $v$ be a solution to

$$
v_{t}=\mathcal{L} v, \quad v(x, 0)=v_{0} .
$$

With the notion of decay character in hand, the following result can be obtained :

Theorem $2.18([13,169])$. Let $v_{0} \in H^{s}\left(\mathbb{R}^{n}\right), s \geq 0$, have decay character $r_{s}^{*}\left(v_{0}\right)=r_{s}^{*}$. Let $v(t)$ be a solution to (40) with data $v_{0}$. Then there exist constants $C_{1}, C_{2}, C_{\epsilon}, C_{m}>0$, so that

1. If $r_{s}^{*} \in\left(-\frac{n}{2}+s, \infty\right) \Rightarrow C_{1}(1+t)^{-\frac{1}{\alpha}\left(\frac{n}{2}+r_{s}^{*}+s\right)} \leq\|v(t)\|_{\dot{H}^{s}}^{2} \leq C_{2}(1+t)^{-\frac{1}{\alpha}\left(\frac{n}{2}+r^{*}+s\right)}$,

2. If $r_{s}^{*}=\infty \Rightarrow\|v(t)\|_{\dot{H}^{s}}^{2} \leq C_{m}(1+t)^{-m}, \quad \forall m>0$,

3. If $r_{0}^{*}=-\frac{n}{2} \Rightarrow\|v(t)\|_{2}^{2} \geq C_{\epsilon}(1+t)^{-\epsilon}, \quad \forall \epsilon>0$,

The above theorem follows combining energy inequalities with estimates resulting from the decay and an appropriate Fourier Splitting. For details when $s=0$ see [13], when $s>0$ see [169]. 
Remark 2.19. For $s>0$, to determine the decay in $\dot{H}^{s}$ of solutions to (40), it is necessary first to establish how the decay character of $\Lambda^{s} u_{0}$ and that of $u_{0}$ are related. Specifically if $u_{0} \in H^{s}\left(\mathbb{R}^{n}\right), s>0$, it can be shown that $r_{s}^{*}\left(u_{0}\right)=s+r^{*}\left(u_{0}\right)$, provided $-\frac{n}{2} \leq r^{*}\left(u_{0}\right) \leq \infty$. For a proof the reader is referred to [169].

The recent paper [24] explores further the relation between the behavior of $\widehat{v}_{0}$ near $\xi=0$ and the decay estimates from above and below. For example, under the same assumptions as before on $\mathcal{L}$, with the additional condition that the symbol $\mathcal{M}(\xi)$ is homogeneous of degree $2 \alpha$, it is proved therein that

$$
\left\{\begin{array}{l}
\liminf _{\rho \rightarrow 0^{+}} \rho^{-2 \sigma} \int_{|\xi| \leq \rho}\left|\widehat{v}_{0}(\xi)\right|^{2} \mathrm{~d} \xi>0 \\
\limsup _{\rho \rightarrow 0^{+}} \rho^{-2 \sigma} \int_{|\xi| \leq \rho}\left|\widehat{v}_{0}(\xi)\right|^{2} \mathrm{~d} \xi<\infty
\end{array} \Longleftrightarrow v_{0} \in \dot{\mathcal{A}}_{2, \infty}^{-2 \sigma}, \Longleftrightarrow(1+t)^{-\frac{\sigma}{\alpha}} \lesssim\|v(t)\|_{2} \lesssim(1+t)^{-\frac{\sigma}{\alpha}} .\right.
$$

Here $\dot{\mathcal{A}}_{2, \infty}^{-2 \sigma}$ is a specific subset of the Besov space $\dot{B}_{2, \infty}^{-2 \sigma}\left(\mathbb{R}^{n}\right)$ that can be defined through suitable size estimates on the dyadic blocks of frequency decompositions (see [24] for more details). The notation $\lesssim$ means that the inequality holds up to a multiplicative constant independent on $t$.

When an $L^{2}$ function satisfy any of the above equivalent conditions (41), the exponent $\sigma \in(0, \infty)$ is uniquely determined. This exponent $\sigma=\sigma\left(v_{0}\right)$ was coined the Besov character of $v_{0}$. It is related to (the improvement made in [24] of) Bjorland-Schonbek's notion of "decay character", by the relation $\sigma\left(v_{0}\right)=r^{*}\left(v_{0}\right)+\frac{n}{2}$.

\subsubsection{Application to the decay for Navier-Stokes}

The decay estimates for the linear system are used to obtain the decay rates for appropriate non linear diffusive systems. In [13] it was used for solutions to the NS equations with zero force. This allows, in particular, to restate the decay results Wiegner's Theorem 2.5 in the following form:

Theorem 2.20 (See [13]). Let $u$ be as in Theorem 2.5 (with forcing term $f \equiv 0$ ). Assume that $u_{0} \in L^{2}\left(\mathbb{R}^{n}\right)$ has a decay character $r^{*}=r^{*}\left(u_{0}\right)$. Then,

1. If $-\frac{n}{2}<r^{*}<1-\frac{n}{2}$ then there are constants $C_{1}, C_{2}, C_{3}>0$ so that

$$
C_{1}(1+t)^{-r^{*}-\frac{n}{2}} \leq\|u(t)\|_{2}^{2} \leq C_{2}\left(C_{3}+t\right)^{-r^{*}-\frac{n}{2}}
$$

2. If $r^{*} \geq 1-\frac{n}{2}$ then $\|u(t)\|_{2}^{2} \leq C(1+t)^{-\frac{n}{2}-\beta}$ where $\beta=\min \left(r^{*}, 1\right)$.

3. If $r^{*}=-\frac{n}{2}$ and $n=3,4$ then $\|u(t)\|_{2}^{2}$ decays slower than any algebraic decay rate for large time.

The first conclusion of the above theorem can be made more precise. In fact, necessary and sufficient conditions for the validity of upper-lower decay estimates can be prescribed. 
Theorem 2.21 (See [24]). Let $u_{0}$ and $u$ as in Theorem 2.5 (with forcing term $f \equiv 0$ ). Let $0<\sigma<5 / 4$. The three following properties are equivalent:

(i) $\liminf _{\rho \rightarrow 0} \rho^{-2 \sigma} \int_{|\xi| \leq \rho}\left|\widehat{u}_{0}(\xi)\right|^{2} d \xi>0 \quad$ and $\quad \limsup _{\rho \rightarrow 0} \rho^{-2 \sigma} \int_{|\xi| \leq \rho}\left|\widehat{u}_{0}(\xi)\right|^{2} d \xi<\infty$,

(ii) $u_{0} \in \dot{\mathcal{A}}_{2, \infty}^{-2 \sigma}$

(iii) $(1+t)^{-\sigma} \lesssim\|u(t)\|_{2} \lesssim(1+t)^{-\sigma}$.

The proof relies on the analysis of the linear system described in the previous subsection and on Z. Skalák's version of the "Inverse Wiegner's theorem", [196].

Applications of the decay character for other diffusive equations will be revisited in Section 5.4.

\subsection{Non-decaying velocities}

When the energy inequality

$$
\int_{0}^{t}\|u(t)\|_{2}^{2}+2 \int_{0}^{t}\|\nabla u\|_{2}^{2} \mathrm{~d} s \leq\left\|u_{0}\right\|_{2}^{2}
$$

is not available, the tools described in Section 2.4 are no longer effective. The case of solutions with non-decaying velocities attracted considerable attention. For example, if one drops the $L^{2}$-condition on the initial datum (that implies some sort of decay at infinity), replacing it with the more general condition $u_{0} \in L_{\text {uloc }}^{2}\left(\mathbb{R}^{3}\right)$ (the space of uniformly locally$L^{2}$ vector fields), then it is still possible to construct weak solutions satisfying a local energy inequality, defined at least on some finite time interval: under mild additional condition such solutions can be extended to global weak solutions (see P. G. Lemarié-Rieusset's book [143] for a detailed account) but nothing is known on their large time behavior.

Some results on the large time behavior of solution arising from $L^{\infty}\left(\mathbb{R}^{2}\right)$ initial data are briefly described here. In any space dimension $n \geq 2$, if $u_{0} \in L^{\infty}\left(\mathbb{R}^{n}\right)$, then it is possible to construct a unique local in time mild solution belonging to the space $B U C$ of bounded and uniformly continuous functions, with the corresponding pressure satisfying the natural condition

$$
p=(-\Delta)^{-1}(\nabla \cdot(u \otimes u)) .
$$

In the two-dimensional case, such solution is in fact global in time. In this case, the main issue is to establish some a priori bound on the growth of the velocity for large times. The problem of its behavior at infinity was first addressed by Y. Giga, S. Matsui, Y. Sawada [95], who established a double exponential bound for the growth of $\|u(t)\|_{\infty}$ and

Y. Sawada and Y. Taniuchi who improved this bound to a simple exponential-in-time growth, see [178]. Their proof relies on the maximum principle for the vorticity equation and splitting the frequencies into low and higher parts. More recently, using in a deeper way the structure of the Navier-Stokes equations, and the local energy inequality, S. Zelik 
(see [208], or Th. Gallay's review [83]) proved that the $L^{\infty}\left(\mathbb{R}^{2}\right)$-norm of $u$ grows at most linearly in time:

$$
\|u(t)\|_{\infty} \leq C\left\|u_{0}\right\|_{\infty}\left(1+C t\left\|u_{0}\right\|_{\infty}^{2}\right)
$$

In this bound, the constant explicitly depend on the viscosity coefficient (is set $=1$ throughout for simplicity) and breaks down in the limit as $\nu \rightarrow 0$. However, when one also assumes that the initial vorticity is in $L^{\infty}$, then these bounds turn out to be independent on the viscosity coefficient.

\subsection{The role of external forces}

The results described in Sections 2.2, 2.3, 2.4 are valid with non zero external forces provided they decay sufficiently fast in $L^{2}$. In [180], it is shown that if

$$
\|f(t)\|_{2} \leq C(1+t)^{-\frac{n}{2}-1} \text { and } f \in L^{\infty}\left((0, \infty), W^{-1,1}\left(\mathbb{R}^{n}\right)\right)
$$

then the energy decays at the same rate as for $f=0$. In this case, the FS method includes an extra term that decays much faster and hence is negligent. A natural question is what happens in the case that the external forces are slowly decaying. The surmise is that the forcing terms will steer the decay to a slower rate.

In [171], T. Ogawa, S.V Rajopadhye, M.E. Schonbek, studied the uniform and nonuniform decay for fluids with slowly decaying forcing terms. They looked for the weakest possible assumptions on $f$ so that a weak solution $u$, constructed by the techniques in $[29,145]$, that complies with the strong energy inequality (5), and satisfies $\|u(t)\|_{2} \rightarrow 0$ as $t \rightarrow \infty$, with or without rate. Technical assumptions on the force are needed, which include the assurance that $f$ is in the dual of the space of the weak solution. The main assumption $(\mathcal{A} 1)$ is quite technical. Instead of giving the exact terms of $(\mathcal{A} 1)$, only some typical forces included under assumption are depicted here :

1. $f \in L^{1}\left((0, \infty), L^{2}\right)$ and $f \in L^{2}\left((0, \infty), L^{\frac{2 n}{n+2}}\right)(n \geq 3)$.

2. If $f$ belongs to appropriate weighted space, then a weaker condition such as $|x| f \in$ $L^{2}\left((0, \infty), L^{2}\right)$, will suffice. For details see [171].

For uniform and non uniform decay a generalization of the strong energy inequality (5) is needed:

Proposition 2.22 (Existence and generalized energy inequality [171]). Let $u_{0} \in L^{2}$ and $f$ satisfy assumption $(\mathcal{A} 1)$. There exists $u \in L^{\infty}\left((0, T), L_{\sigma}^{2}\right) \cap L^{2}\left((0, T), \dot{H}_{\sigma}^{1}\right)$ for all $T>0$, a weak solution of $N S$ with datum $u_{0}$, satisfying (5). For any $E(t) \in C^{1}\left(\mathbb{R}_{1} \mathbb{R}_{+}\right)$with $E(t) \geq 0$ and $\psi \in C^{1}\left(\mathbb{R} ; C^{1} \cap L^{2}\right)$, u satisfies the generalized energy inequality (GE)

$$
\begin{array}{r}
E(t)\|\psi(t) * u(t)\|_{2}^{2} \leq E(s)\|\psi(s) * u(s)\|_{2}^{2}+\int_{s}^{t} E^{\prime}(\tau)\|\psi(\tau) * u(\tau)\|_{2}^{2} d \tau \\
+2 \int_{s}^{t} E(\tau)\left|\left\langle\psi^{\prime}(\tau) * u(\tau), \psi(\tau) * u(\tau)\right\rangle-\|\nabla \psi(\tau) * u(\tau)\|_{2}^{2}\right| d \tau \\
+2 \int_{s}^{t} E(\tau)(|\langle u \cdot \nabla u(\tau), \psi(\tau) * \psi(\tau) * u(\tau)\rangle|+|\langle f(\tau), \psi(\tau) * \psi(\tau) * u(\tau)\rangle|) d \tau
\end{array}
$$


where $0 \leq s<t<\infty$, for almost all $s$ and, all $t$.

Non-uniform decay. The Fourier splitting method, combined with inequality (44) yields the desired decay. As in [156], for the non-uniform decay, one decomposes the solution into low and high frequency modes and considers them separately. Choose $\phi=\phi(\xi)=e^{-|\xi|^{2}}$ :

$$
\|u(t)\|_{2}^{2}=\|\phi u(t)\|_{2}^{2}+\|(1-\phi) u(t)\|_{2}^{2}=\mathcal{L}(t)+\mathcal{H}(t)
$$

The decay of $\mathcal{L}(t)$ follows by technical Sobolev inequalities. For the decay of $\mathcal{H}(t)$ a modified FS technique is used. Choose $\psi(t)=1-\phi$. Split the frequency space into

$$
\mathbb{R}^{n}=\mathcal{S}(t) \cup \mathcal{S}(t)^{c}, \quad \text { with } \mathcal{S}(t)=\{\xi:|\xi|<\mathcal{G}(t)\} .
$$

Choose $E(t)=(t+1)^{\alpha}, \mathcal{G}^{2}(t)=\frac{\alpha}{2(t+1)}$. Then $(E(t), \mathcal{G})$ satisfy $E^{\prime}(t)-2 \mathcal{G}^{2}(t) E(t)=0$. Here $\alpha$ depends only on the dimension. Note that the third term on the right hand side of (44) vanishes since $\psi^{\prime}=0$. Combining the second term with the diffuse fourth, yields that it is only necessary to estimate the solution on a small ball of shrinking radius $\mathcal{G}(t)=\frac{\alpha}{\sqrt{2(t+1)}}$. Assumption $\mathcal{A}_{1}$ allows the term involving the force to be estimated by a Hardy inequality.

Uniform decay. For the uniform decay more assumptions on the force are needed. Here one uses directly a FS-technique combined with the generalized inequality (44). For details see $[171]$.

For the influence of external forces on the point-wise decay, the reader may consult the results by Bae and Brandolese in [5].

\section{Decay results in more general domains}

The literature of this subject is huge, the presentation in this chapter will be restricted to a few of the many relevant results. H. Sohr's book [197] is a classical reference for this topic. The reader is also referred to the work by R. Farwig, H. Kozono and J. Sohr [66] for more recent developments about domains with non-compact boundaries. The contributions in this handbook by M. Geißert, M. Hieber, J. Saal [111] and S. Monniaux, Z Shen [166] give also up-to-date results.

\subsection{Decay in bounded domains}

For bounded domains existence and stability has been understood at several levels. One advantage over unbounded domains is the validity of the Poincaré inequality. In two and three dimensions, it is known since Leray and Hopf's work [144,145] that the $L^{2}$ norm of the solution and its corresponding enstrophy decays at least exponentially as time tends to infinity. In [71] Foias and Saut show that the decay is exactly exponential, in the sense that the limit of the ratio of the enstrophy over the energy tends to an eigenvalue of the Stokes operator. This result serves as basis to obtain asymptotic expansion of the solution 
to NS as $t \rightarrow \infty$. More information on the asymptotic behavior of solutions in bounded domains can be found in $[60,197]$.

An original approach is that of [167], where S. Nečasová and P. Rabier make a careful analysis of the relationship between the time decay of solutions of the Navier-Stokes system in a bounded domain and the time decay of the external force $f$. They show that the decay properties of the solution depend upon the type and amount of decay of $f$ and upon the function spaces to which the data belong, but not on $f$ or of the initial data themselves. The "type" (exponential, power-like, etc.) and the "amount" of the decay of $f$ are defined in a rigorous way by considering the class of functions $\rho=\rho(t)$ such that, for some $s>0$, $e^{s \rho} f$ belongs to an $L^{p}\left(\mathbb{R}^{+}, L^{q}(\Omega)\right)$ space. In the same way, the decay of the solution is measured by the validity of conditions of the form $e^{s \rho} u \in L^{p}\left(\mathbb{R}^{+}, L^{q}(\Omega)\right)$. A consequence of the main result of [167], is that the solution will always inherit power-like decays of the external forces, but only part of their exponential decays, in a sense made precise in [167].

\subsection{Decay in exterior domains}

Exterior domain problems are used to investigate the behavior of fluids around an obstacle. One natural question to ask is what is the influence of the obstacle in the decay rates. Focus here is centered on the fundamental work of Borchers and Miyakawa [14-17]. Historically the analysis begins with the results by Heywood in [106,107], where the question was to compare the behavior of the stationary and the non-stationary solutions to the NS equations. In [141] Ladyzhenkaya states that "it seems impossible to prove the a priori estimates required to investigate for all $t>0$ a non-stationary flow past an object which begins at $t=0$ as a perturbation of one of these stationary solutions". An important element that allowed to overcome the obstacles specified in [141] is the work by Finn [68-70] on stationary flows, in exterior domains. Briefly, in [107] the following stability result was established:

Let $u=u(x, t)$ be the solution to NS in an exterior domain $\Omega \subset \mathbb{R}^{3}$ outside of a bounded domain with smooth boundary $\Sigma$. Suppose the forcing term is only $x$-dependent and small. Let $w=w(x)$ be the solution to the corresponding stationary problem. Suppose that $u=w$ on $\partial \Omega, t \geq 0$. In addition there are some appropriate somewhat involved conditions on $u_{0}=u(x, 0)$ and $w$, for details see [107]. If $\mathcal{A}$ is any bounded subset of $\Omega$, then

$$
\lim _{t \rightarrow \infty}\|\nabla v(t)-\nabla w\|_{L^{2}(\Omega)}=0, \quad \lim _{t \rightarrow \infty}\|v(t)-w(x)\|_{L^{2}(\mathcal{A})}=0 .
$$

This result was extended by Masuda [155] giving the first $L^{2}$ - rate of decay result for non stationary solutions to NS in an exterior domain $\Omega$. The domain $\Omega$ is as in Heywood's papers $[106,107]$, and $v, w$ satisfy the conditions (1) and (2) above. Then in [155] it is shown that

$$
\sup _{x \in \Omega}|v(x, t)-w(x)| \leq M t^{-\frac{1}{8}}, \quad \text { for } t \geq T_{0}>0, \text { and some constant } M .
$$

As Heywood points out in [105] "It was recognized by Masuda that this order of decay might be improved through the use of sharper estimates for solutions of the Stokes problem." 
The decay results in [155] were ameliorated in [105]. The optimal rates with less conditions on the exterior region were finally obtained in a series of papers by W. Borchers and T. Miyakawa [14-16]. These results involve a deep analysis of properties of $A_{r}$, (the Stokes operator in $L^{r}$ ) with fractional powers. In particular a fundamental Sobolev embedding theorem for fractional powers of the Stokes operators in exterior domains is obtained. The notation used is as follows:

$$
A u=A_{r} u=-P_{r} \Delta u, \quad u \in D\left(A_{r}\right)=L_{\sigma}^{r}(\Omega)^{r} \cap H_{0}^{1, r}(\Omega) \cap H^{2, r}(\Omega) .
$$

Here $\Omega$ is a domain in $\mathbb{R}^{n}, L_{\sigma}^{r}(\Omega)$ are solenoidal vector functions in $L^{r}$ and, $P_{r}$ is the bounded projection from $L^{r}(\Omega)$ into $L_{\sigma}^{r}(\Omega)^{r}$. The exterior domain $\Omega$ under consideration, is the complement of a finite number of bounded domains with $C^{\infty}$ boundaries. The forcing term is zero. The main theorems for solutions $v(x, t), x \in \Omega, t>0$ obtained in [14] are as follows:

Theorem $3.1([14])$. Let $n \geq 3$. Let the initial data $u_{0} \in L_{\sigma}^{2}(\Omega)$, let $v^{0}(t)$ be the solution to the Stokes problem with data $u_{0}$ and $\left.v^{0}(t)\right|_{\partial \Omega}=0, v^{0} \rightarrow 0$ as $|x| \rightarrow \infty$. Then

(i) There is a weak solution $v$ of $N S$ which satisfies $\|v(t)\|_{2} \rightarrow 0$ as $t \rightarrow \infty$.

(ii) If in addition $\left\|v^{0}(t)\right\|_{2}=O\left(t^{-\alpha}\right)$ as $t \rightarrow \infty$, for some $\alpha>0$, then $\|v(t)\|_{2}=O\left(t^{-\beta}\right)$ as $t \rightarrow \infty$ with $\beta=\min \left\{a, \frac{n}{4}-\epsilon\right\}$, for some $\epsilon \in\left(0, \frac{1}{4}\right)$.

(iii) $\left\|v(t)-v^{0}(t)\right\|_{2}=o\left(t^{\left.-\frac{n}{4}+\frac{1}{2}\right)}\right)$ as $t \rightarrow \infty$.

(iv) If in addition $\left\|v^{0}(t)\right\|_{2}=O\left(t^{-\alpha}\right)$ as $t \rightarrow \infty$, for some $\alpha>0$, then $\left\|v(t)-v^{0}(t)\right\|_{2}=$ $O\left(t^{-\gamma}\right)$ ast $\rightarrow \infty, \gamma=\frac{n}{4}-\frac{1}{2}+\alpha$, if $\alpha<\frac{1}{2}$ and $0<\gamma<\frac{n}{4}$ if $\alpha \geq \frac{1}{2}$.

If a weak solution satisfies the energy inequality (5) with $f=0$, then $v$ has all the previous properties.

Main ideas for the proof: The spectral decomposition of the self-adjoint operator $A_{2}$ given by

$$
A_{2}=\int_{0}^{\infty} \lambda d E_{\lambda}
$$

is used to obtain a lower bound for the $L^{2}$ norm of the gradient of the solution to NS.

$$
\|\nabla v\|_{2}^{2}=\left\|A_{2}^{\frac{1}{2}} v\right\|_{2}^{2}=\int_{0}^{\infty}\left\|E_{z} v\right\|_{2}^{2} \mathrm{~d} z \geq \int_{\lambda}^{\infty}\left\|E_{z} v\right\|_{2}^{2} \mathrm{~d} z \geq \lambda\left(\|v\|_{2}^{2}-\left\|E_{\lambda} v\right\|_{2}^{2}\right)
$$

This approach depicts Fourier splitting using spectral theory. The idea was already used in [118]. Estimate (45) combined with the energy inequality (5) yields

$$
\|v(t)\|_{2}^{2}+\int_{s}^{t} \lambda(\tau)\|v(\tau)\|_{2}^{2} \mathrm{~d} \tau \leq\|v(s)\|_{2}^{2}+\int_{s}^{t} \lambda(\tau)\left\|E_{\lambda} v(\tau)\right\|_{2}^{2} \mathrm{~d} \tau .
$$

Bounds for the Stokes operator with fractional powers combined with (46) yields an inequality for $\left\|E_{\lambda} v(t)\right\|_{2}$ where a Gronwall estimate can be applied. This provides the 
decay expected for hypothesis (i) and (ii). Cases (iii) and (iv) are more involved: in particular it is necessary to analyze $w=v(t)-v^{0}(t)$. As stated in [14], if $u_{0} \in L^{r} \cap L_{\sigma}^{2}$ one can take $\alpha=\left(\frac{n}{q}-\frac{n}{2}\right) / 2$ and thus $\alpha=\beta$. The final theorem in [14] gives decay in $L^{q}$, $q<2$ for solutions with data in $u_{0} \in L^{r} \cap L_{\sigma}^{2}, r \in(1, n / n-1]$ and $r<\frac{2 n}{n+2}$. The decay obtained is $\|v(t)\|_{q}=o\left(t^{\eta}\right)$, as $t \rightarrow \infty$, with $\eta=\left(\frac{n}{q}-\frac{n}{q}\right) / 2$.

Papers $[15,16]$ improve the results in [14]. The main difference in [15] is that less conditions are required on the data to get the decay. In [15] the comparison is with the solutions of the underlying heat equation. One of the main Theorems in [15] states

Theorem 3.2 ([15]). Let the initial data $u_{0} \in L^{r}(\Omega) \cap L_{\sigma}^{2}(\Omega)$ for some $1<r<2$, then there is a weak solution $v$ of $N S$ such that $\|v(t)\|_{2}=O\left(t^{-\gamma}\right)$, as $t \rightarrow \infty$, where $\gamma=\frac{1}{2}\left(\frac{n}{q}-\frac{n}{2}\right)$. The same holds for solutions which satisfy the energy inequality (5) (here $f=0)$.

The results in [16] involve arbitrary unbounded domains in dimensions $n=2,3,4$ where the Poincaré inequality might not hold. The methods used are based on the ones in the former two papers.

Remark 3.3. The last Theorem in [14] includes the main result of Maremonti [153]. Moreover this theorem also ameliorates the work of Galdi and Maremonti [79] and Maremonti [154].

The work of Borchers-Miyakawa, [14-16] dealt with solutions to NS for dimensions $n \geq 3$. The work of Kozono and Ogawa $[130,131]$ investigates questions related to decay in two dimensions. Specifically in [131] solutions to NS are studied for initial data in $L_{\sigma}^{2}(\Omega)$. Uniqueness and existence of a global strong solution $u=u(x, t)$ are first established. Decay rates obtained for $u$ and $\frac{d u}{d t} \in L^{p}, 2 \leq p<\infty$ are optimal in the sense that they coincide with the rates of the solutions to the underlying linear equations. The $L^{\infty}$ rates for $u$ and $\frac{d u}{d t}$ differ from the optimal one by a logarithmic multiple. Rates of decay for the $L^{2}$ norms of $A^{\alpha} u, 0<\alpha \leq 1$ and of $A^{\alpha}\left(\frac{d u}{d t}\right), 0<\alpha<1$ are also obtained.

The results on decay in [131] follow by an analysis of the operator $\left(A_{2}+\lambda\right)^{-\alpha}, 0<\alpha \leq$ $\frac{1}{2}$. The main difficulty, that the authors have to handle, is that $A_{2}$ in unbounded domains in $\mathbb{R}^{2}$ is not boundedly invertible. The work in [131] extends the one in [130]. In [131] for data with additional conditions, new rates are obtained for $u, \frac{d u}{d t}$, in $L^{p}, 2 \leq p \leq \infty$ and in $L^{2}$ for $A^{\alpha} u, A^{\alpha}\left(\frac{d u}{d t}\right)$. Furthermore decay rates in $L^{p}, 1<p<2$, are derived for solutions with data in $L^{p} \cap L^{2}, 1<p<2$.

In [132] Kozono and Ogawa analyze in exterior domains in $\mathbb{R}^{n}, n \geq 3$, the stability of the stationary solutions and first derivatives in $L^{p}(\Omega)$, with appropriate $p$ depending on conditions on the data and on the stationary solutions.

The decay in weighted spaces also attracted a considerable attention. For a nice review of recents results the the reader is referred to the introduction of the paper [103]. In this paper, C. He and T. Miyakawa address the problem of the time decay of weighted norms 
of weak and strong solutions to the NS equations in a 3D exterior domain. They prove that weak solutions satisfy, for all $t \geq 0$ :

$$
\left\||x|^{\alpha} u(t)\right\|_{2}^{2}+\int_{0}^{t}\left\||x|^{\alpha} \nabla u(\tau)\right\|_{2}^{2} d \tau \leq c \quad\left(1<\alpha<\frac{n}{2}\right)
$$

and

$$
\left\||x|^{\beta} u(t)\right\|_{2} \leq c(1+t)^{-\frac{n(\alpha-\beta)}{4 \alpha}} \quad\left(0 \leq \beta \leq \alpha<\frac{n}{2}\right) .
$$

In addition, the authors find the optimal decay rates in space-time of strong solutions. The balance between these two kinds of decays turns out to be similar to that of solutions of the Cauchy problem. One important issue is to know if the above range on the parameters $\alpha$ and $\beta$ is the best possible. In this regard, necessary and sufficient conditions for the improvement of such a range are discussed. These conditions involve the vanishing of the total net force exerted by the fluid and are related to $\mathrm{H}$. Kozono $L^{1}$-summability problem of the velocity field. It is difficult to check if the solutions satisfy such conditions or not. However, the authors prove that it is indeed the case for flows with special symmetries.

Up-to-date information on the asymptotic behavior in exterior domains, with focus on weighted-spaces, higher-order norms, can be found in P. Han's recent paper [100]. Han's arguments are effective to obtain also subtle $L^{1}$-estimates, provided the initial data are taken in appropriate subspaces of $L_{\sigma}^{1}(\Omega)$.

\section{Convergence and stability results for stationary solutions}

The decay results described in Sections 2.2-2.4, can be viewed as asymptotic stability results of the identically zero solutions. In the presence of an appropriate time-independent external force $f$, it is possible to construct a unique nonzero stationary solution $w$. Then the study of the large time decay of a perturbed problem provides information on the asymptotic stability of such solutions. In this section a few of the many important results on the convergence to stationary solutions are recalled, referring to G. Galdi [76], T. Hishida [113], and K. Pileckas [152] contributions to this handbook for a more complete presentation.

\subsection{Some results on the asymptotic stability of stationary solutions of the Navier-Stokes equations}

\subsubsection{Stability of stationary flows in $L^{p}\left(\mathbb{R}^{n}\right), p \geq n$, and in exterior domains}

When $f$ is an appropriate forcing term of the form $f(x)=\nabla \cdot F(x)$, it is possible to construct a unique nonzero stationary solution $w$, i.e. a solution of the system

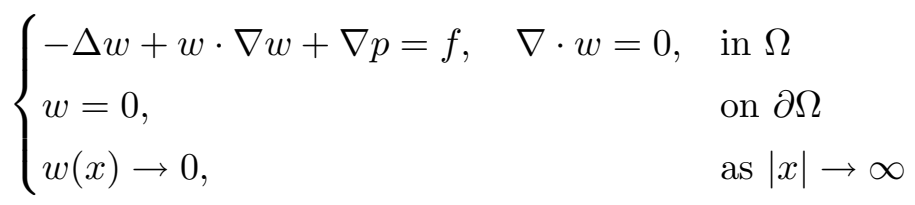


where $\Omega$ is an exterior domain of $\mathbb{R}^{n}, n \geq 3$. The underlying linear system is the Stokes system. An alternative realistic boundary condition at infinity is $\lim _{|x| \rightarrow \infty} w(x)=w_{\infty} \in$ $\mathbb{R}^{n}$, where $w_{\infty}$ is a constant vector field. If $w_{\infty} \neq 0$ then the underlying linear system is no longer given by the Stokes system, but by the Oseen system. For the Oseen case, stability results can be found in Maremonti's work in [153]. The stability results in [153] work for small perturbations of solutions to the stationary solutions $w$ in the whole space and exterior domains. For more details on stability in the Oseen case the reader is referred to [77]. General information on properties of the Stokes operator can be found in the contribution to this Handbook by M. Hieber and J. Saal [111]. In the remaining part of this section the case $w_{\infty}=0$ is discussed.

For the system (47), W.Borchers and T. Miyakawa [17] under a smallness assumption of the form

$$
\sup _{x \in \Omega}|x|^{2}|F(x)|+\sup |x|^{3}|\nabla F(x)|<\epsilon
$$

constructed such a solution $w$, satisfying

$$
\sup _{x \in \Omega}|x||w(x)|+\sup |x|^{2}|\nabla w(x)|<\infty,
$$

the uniqueness being guaranteed if the left-hand side of the above inequality is small. The result of [17] also asserts that if such solution $w$ is perturbed by a disturbance " $a$ " (assumed to be small in the $L^{n, \infty}(\Omega)$ norm), then the solution $u$ of the Navier-Stokes equations on $\Omega$ with homogeneous boundary conditions and external force $\nabla \cdot F$, arising from $u_{0}=w+a$, satisfies

$$
\|u(t)-w\|_{L^{p}(\Omega)}=\mathcal{O}\left(t^{-\frac{n}{2}\left(\frac{1}{n}-\frac{1}{p}\right)}\right), \quad\|\nabla u(t)-\nabla w\|_{L^{n}(\Omega)}=\mathcal{O}\left(t^{-\frac{1}{2}}\right)
$$

as $t \rightarrow \infty$, for all $n \leq p<\infty$.

It is possible to considerably relax the condition on the initial force in order to get the existence and uniqueness of a solution $w$ of (47) in a suitable class as well as the convergence toward the stationary solution. For example, H. Kozono and M. Yamazaki [135] achieved this by simply assuming that $F$ is small in the Lorentz space $L^{\frac{n}{2}, \infty}(\Omega)$, with no needed condition on its gradient (in this case $w$ belongs to $L^{n, \infty}(\Omega)$ ).

\subsubsection{Stability of stationary flows in $\mathbb{R}^{3}$ in $L^{p}, p<3$}

A different approach, for the stability problem in the whole $\mathbb{R}^{3}$ has been obtained by C. Bjorland and M.E. Schonbek [12], with the motivation of getting stability results in the $L^{2}$-norm. For stationary solutions $w$ of (47) with appropriate forcing terms it is easy to see that $\|\nabla w\|_{2}<\infty$. It is more difficult to show that the $L^{2}$-norm of the solutions is finite: this usually requires some smallness assumption. To insure such an $L^{2}$ bound, in [12], it was assumed that the forcing terms have vanishing frequencies in a neighborhood of the origin and that the product $\|f\|_{2}\|f\|_{H_{\sigma}^{-1}}$ is small. This allowed to establish the existence of a unique stationary solution that is asymptotically stable in $L^{2}$ under small initial perturbations. The proof involves a modified FS technique applied to the solution 
of the equations for $z=u-w$, where $\mathrm{u}$ is the solution to NS and, $w$ is the solution to the stationary NS.

Wider stability results were obtained in [10] by C. Bjorland, L. Brandolese, D. Iftimie and M.E. Schonbek, where the stability for $L^{p}$ spaces, $3 / 2<p \leq \infty$ were considered. These results improved the work in [12] in the case $p=2$, by relaxing the assumptions on $f$. Specifically, the stability of the stationary solution $w$ was investigated in the $L^{p}$ and the Lorentz $L^{p, \infty}$-norms. Stationary solutions $w \in L^{3, \infty} \cap L^{p}$ or $w \in L^{3, \infty} \cap L^{p, \infty}$, small in $L^{3, \infty}$ are investigated. These authors show that possibly initially large $L^{3, \infty}$ non-stationary solutions eventually become small in $L^{3, \infty}$ (i.e. they eventually become of a size comparable to that of $w$ ). Decay estimates for the difference $u(t)-w$ are then established and a necessary and sufficient conditions are given to have the convergence result $u(t) \rightarrow w$.

Results in the same spirit as in [10], but under even less restrictive assumptions on $f$ were found by T.V. Phang and N.C. Phuc [174]. Let us describe in more details the stability result of [174] that seems to be optimal, in the sense that it requires the weakest conditions on the external force $f$ known so far. As pointed out in [10], in order to construct $w \in L_{l o c}^{2}$ (this is needed e.g. to give a meaning to $\nabla \cdot(w \otimes w)$ as a distribution) it is tempting to construct $w$ in the largest space that is both translation and scale invariant, embedded in $L_{l o c}^{2}$. Such space is known to be the Morrey-Campanato space $\mathcal{M}_{2,2}$ : the weakest possible smallness assumption under which one could hope to construct such solution $w \in \mathcal{M}_{2,2}$ would then be of the form $\left\|(-\Delta)^{-1} f\right\|_{\mathcal{M}_{2,2}}<\epsilon$. But, in fact, such a construction is still an open problem. A good substitute for the space $\mathcal{M}_{2,2}$ is the space

$$
\mathcal{V}_{1,2}=\left\{w \in L_{l o c}^{2}:\|f\|_{\mathcal{V}_{1,2}} \equiv \sup _{K \subset \subset \mathbb{R}^{n}}\left(\int_{K}|f|^{2}\right) / \operatorname{cap}_{1,2}(K)\right\}
$$

where $\operatorname{cap}(K)_{1,2}$ is the capacity of the compact $K$ (see [174]). As a matter of fact, $\mathcal{V}_{1,2}$ is only slightly smaller than $\mathcal{M}_{2,2}$ :

$$
L^{n, \infty}\left(\mathbb{R}^{n}\right) \subset \mathcal{M}_{2+\epsilon, 2+\epsilon} \subset \mathcal{V}_{1,2} \subset \mathcal{M}_{2,2},
$$

where the norms of all the spaces in the above chain of inclusions share the scaling property $\|w\|=\|\lambda w(\lambda \cdot)\|$. The results of [174] can be summarized as follows: there exist an absolute constant $\delta>0$ such that:

- if $\left\|\Delta^{-1} f\right\|_{\mathcal{V}_{1,2}}<\delta$, then there is one unique solution to (47) such that $\|w\|_{\mathcal{V}_{1,2}} \simeq$ $\left\|\Delta^{-1} f\right\|_{\mathcal{V}_{1,2}}$.

- If $u_{0}$ is a divergence-free vector field such that $\left\|u_{0}-w\right\|_{\mathcal{V}_{1,2}} \leq \delta$, then there is a unique solution $u$ of the non-stationary Navier-Stokes equation (47) such that $\left\|(-\Delta)^{s / 2} u(t)-w\right\|_{\mathcal{V}_{1,2}} \simeq t^{-s / 2}\left\|u_{0}-w\right\|_{\mathcal{V}_{1,2}}$, for all $-1 \leq s<1$. (The case $s=0$ provides the Lyapunov stability of $w)$. 


\section{2 $\quad L^{2}$-perturbations of infinite energy flows}

\subsubsection{Perturbation of Landau and of more general solutions}

The work in $[111,119,120]$. analyzes the asymptotic behavior of infinite energy solutions to NS, under $L^{2}\left(\mathbb{R}^{3}\right)$ perturbations. The results in [119] by G. Karch and D. Pilarczyk address the global asymptotic stability of the family of axisymmetric stationary solutions introduced by Landau in [142]:

$$
\begin{aligned}
v_{c}^{1}(x) & =2 \frac{c|x|^{2}-2 x_{1}|x|+c x_{1}^{2}}{|x|\left(c|x|-x_{1}\right)^{2}}, & v_{c}^{2}(x) & =2 \frac{x_{2}\left(c x_{1}-|x|\right)}{|x|\left(c|x|-x_{1}\right)^{2}}, \\
v_{c}^{3}(x) & =2 \frac{x_{3}\left(c x_{1}-|x|\right)}{|x|\left(c|x|-x_{1}\right)^{2}}, & p_{c}(x) & =4 \frac{c x_{1}-|x|}{|x|\left(c|x|-x_{1}\right)^{2}} .
\end{aligned}
$$

Here $|x|=\sqrt{x_{1}^{2}+x_{2}^{2}+x_{3}^{2}}$ and $c$ is an arbitrary constant such that $|c|>1$. It is well known that in the whole $\mathbb{R}^{3}, v_{c}=\left(v_{c}^{1}, v_{c}^{2}, v_{c}^{3}\right)$, is a distributional stationary solution to NS with a forcing term $f=\left(b(c) \delta_{0}, 0,0\right)$. The parameter $b \neq 0$ depends on $c$ and, $\delta_{0}$ is the Dirac measure. To study the stability it is necessary to analyze the perturbed problem

$$
\begin{aligned}
w_{t}-\Delta w+(w \cdot \nabla) w+(w \cdot \nabla) v_{c}+\left(v_{c} \cdot \nabla\right) w+\nabla \pi & =0, \\
\operatorname{div} w & =0, \\
w(x, 0) & =w_{0}(x) .
\end{aligned}
$$

where $w(x, t)=u(x, t)-v_{c}(x)$ and $u=u(x, t)$ is a solution of the NS system with the external force $f=\left(b(c) \delta_{0}, 0,0\right)$ and, initial datum $u_{0}=v_{c}+w_{0}$. Here $\pi$ is the difference of the pressures. The first step is to obtain an energy inequality:

Theorem 4.1 ([119]). For every $c \in \mathbb{R}$ such that $|c| \geq c_{0}>1$, every $w_{0} \in L_{\sigma}^{2}\left(\mathbb{R}^{3}\right)$, and for every $T>0$ the perturbed problem has a weak solution in the usual energy space which satisfies the strong energy inequality

$$
\|w(t)\|_{2}^{2}+2(1-K(c)) \int_{s}^{t}\|\nabla \otimes w(\tau)\|_{2}^{2} d \tau \leqslant\|w(s)\|_{2}^{2}
$$

for almost all $s \geqslant 0$ including $s=0$ and all $t \geqslant s$. Moreover,

$$
\lim _{t \rightarrow \infty}\|w(t)\|_{2}=0
$$

Ideas of the proof. The energy inequality follows after multiplying the equation by $w$ and integrating. Note that the convective term can be estimated by a Hardy type inequality of the form:

$$
\left|\int_{\mathbb{R}^{3}}(w \cdot \nabla) v_{c} \cdot w d x\right|=\left|\int_{\mathbb{R}^{3}} w \cdot(w \cdot \nabla) v_{c} d x\right| \leqslant K(c)\|\nabla \otimes w\|_{2}^{2}, \quad \forall w \in \dot{H}^{1}\left(\mathbb{R}^{3}\right)^{3},
$$

where $K=K(c)>0, \lim _{|c| \rightarrow 1} K(c)=+\infty$ and $\lim _{|c| \rightarrow+\infty} K(c)=0$. Hence, there exists $c_{0}>1$ such that $K(c)<1$ for all $c$ satisfying $|c| \geqslant c_{0}>1$. 
The next step for the stability is the study of the linearized equation. For this consideration is given to the linearized operator $\mathcal{L}$ which satisfies

$$
z_{t}+\mathcal{L} z=z_{t}-\Delta z+\mathbb{P}\left((z \cdot \nabla) v_{c}\right)+\mathbb{P}\left(\left(v_{c} \cdot \nabla\right) z\right)=0
$$

It is shown that both operators $-\mathcal{L}$ and $-\mathcal{L}^{*}$ are infinitesimal generators of strongly continuous semigroups of linear operators on $L_{\sigma}^{2}\left(\mathbb{R}^{3}\right)$ which are holomorphic in a sector $\{s \in \mathcal{C}:|\operatorname{Arg} s|<\epsilon\}$ for a certain $\epsilon=\epsilon(c)>0$, provided $|c|$ is sufficiently large.

The estimates on $\mathcal{L}$ allow to get the decay of $\left\|e^{-\mathcal{L} t} z_{0}\right\|_{2}$, for $z_{0} \in L_{\sigma}^{2}\left(\mathbb{R}^{3}\right)$. With this information in hand, the decay of $\|w(t)\|_{2}$ follows now from an analysis that involves the integral form of the solution.

The stability, under $L^{2}$-perturbations, of infinite energy solutions $V(x, t)$ other than the Landau ones, is investigated in [120] by G. Karch, D. Pilarczyk and M. Schonbek. One main assumption for the solutions $V=V(x, t)$ under consideration in [120], is that the corresponding convective term satisfies a Hardy type inequality. More precisely it is required that

- $V=V(x, t)$ is a global-in-time solution to NS with an external force $f=f(x, t)$ and an initial datum $V(x, 0)=V_{0}(x)$.

- there exists a Banach space $\left(\mathcal{X},\|\cdot\|_{\mathcal{X}}\right)$ such that this solution satisfies

$$
\begin{aligned}
& -V \in C_{w}([0, \infty], \mathcal{X}), \\
& -\left|\int_{\mathbb{R}^{3}}(g \cdot \nabla) h \cdot V(t) d x\right| \leqslant K \sup _{t>0}\|V(t)\|_{\mathcal{X}}\|\nabla \otimes g\|_{2}\|\nabla \otimes h\|_{2}, \quad \text { some } K>0, \\
& -\forall g, h \in \dot{H}_{\sigma}^{1}\left(\mathbb{R}^{3}\right), \quad K \sup _{t>0}\|V(t)\| \mathcal{X}<1 .
\end{aligned}
$$

Remark 4.2. Spaces ( $\mathcal{X}$ that satisfy the Hardy type inequalities as depicted above, include the Lebesgue and weak-Lebesgue spaces $L_{\sigma}^{3}\left(\mathbb{R}^{3}\right)$ and $L^{3, \infty}\left(\mathbb{R}^{3}\right)$. Other possible choices are $\mathcal{X}=\left\{f \in L_{l o c}^{1}\left(\mathbb{R}^{3}\right):\|f\|=\sup _{x \in \mathcal{X}}|x||f(x)|<\infty\right\}$, or $\mathcal{X}=\mathcal{P} \mathcal{M}^{2}\left(\mathbb{R}^{3}\right)=\left\{v \in \mathcal{S}^{\prime}\left(\mathbb{R}^{3}\right):\right.$ $\left.\operatorname{ess}_{\sup } \operatorname{se\mathbb {R}}^{3}|\xi|^{2}|\hat{v}(\xi)|<\infty\right\}$.

Let $X_{T}=C_{w}\left([0, T], L_{\sigma}^{2}\left(\mathbb{R}^{3}\right)\right) \cap L^{2}\left([0, T], \dot{H}_{\sigma}^{1}\left(\mathbb{R}^{3}\right)\right)$. The following stability result is established in [120].

Theorem 4.3 ([120]). Let $V=V(x, t)$ be a solution to $N S$ satisfying the conditions listed above, with data $V_{0} \in \mathcal{X}$. Then

- $\forall w_{0} \in L_{\sigma}^{2}\left(\mathbb{R}^{3}\right)$, there exists a global-in-time distributional $u=u(x, y)$ solution to $N S$ system with data $u_{0}=V_{0}+w_{0}$ and force $f(x, t)$.

- The difference $w=u(x, t)-V(x, t) \in X_{T}, \forall T>0$, and $w$ satisfies

$$
\|w(t)\|_{2}^{2}+2\left(1-K \sup _{t>0}\|V(t)\|_{\mathcal{X}}\right) \int_{s}^{t}\|\nabla \otimes w(\tau)\|_{2}^{2} d \tau \leqslant\|w(s)\|_{2}^{2},
$$

for almost all $s \geqslant 0$, including $s=0$ and all $t \geqslant s$ 


$$
\text { - }\|u(t)-V(t)\|_{2} \rightarrow 0 \quad \text { as } \quad t \rightarrow \infty .
$$

Comments regarding the proof. For the algebraic decay the high and low frequencies of the $L^{2}$ of norm of $w$ are analyzed separately. For the high frequencies the decay is obtained establishing a generalized energy inequality (similar to the one from Proposition 2.22) combined with a modified Fourier splitting. The low frequencies decay follows by technical energy estimates.

The stable solutions $V$ in [120] include the Landau solutions [119]. Other examples of asymptotically stable solutions $V$, under arbitrary large $L^{2}$-perturbations, are suitable stationary Navier-Stokes equations and Leray-type self-similar solutions. The results in [120] give only non-uniform stability in $L^{2}$. For algebraic rates of decay additional conditions had to be added on the solutions $V$. This is the task done in [111] by T. Hishida and M. Schonbek. To describe the results of [111], we first define the the following space and energy inequality:

$$
\mathcal{V}=V \in L^{\infty}\left(\mathbb{R}_{+} ; L^{n, \infty}\left(\mathbb{R}^{n}\right)\right) \cap C_{w}\left([0, \infty): L^{n, \infty}\left(\mathbb{R}^{n}\right), \quad \operatorname{div} V=0,\right.
$$

where $C_{w}\left([0, \infty) ; L^{n, \infty}\left(\mathbb{R}^{n}\right)\right)$ consists of all weak*-continuous functions with values in $L^{n, \infty}\left(\mathbb{R}^{n}\right)$. Let $w$ be the weak solutions of

$$
\begin{aligned}
w_{t}-\Delta w+(w \cdot \nabla) w+(w \cdot \nabla) V+(V \cdot \nabla) w+\nabla \pi & =0 \\
\operatorname{div} w & =0 \\
w(x, 0) & =w_{0}(x),
\end{aligned}
$$

which satisfies the energy inequality given by

$$
\|w(t)\|_{2}^{2}+2 \int_{s}^{t}\|\nabla w\|_{2}^{2} d \tau \leq\|w(s)\|_{2}^{2}+2 \int_{s}^{t}|\langle V \otimes w, \nabla w\rangle| d \tau
$$

for a.e. $s \geq 0$, including $s=0$, and all $t \geq s$.

With these definitions in hand the following result of [111] is recalled:

Theorem 4.4 ([111]). Let $n=3,4$. Let $w_{0} \in L_{\sigma}^{2}\left(\mathbb{R}^{n} \cap L^{q}\left(\mathbb{R}^{n}\right), q \in[1,2)\right.$, suppose $V(x, t) \in \mathcal{V}$ satisfies $\|V\|=\sup _{t}\|V(t)\|_{3, \infty} \leq \delta$ with $\delta$ small enough. Let $u$ be the solution to NS with data $w_{0}+V, w=u-V$ be a weak solution to the perturbed problem given by (51) satisfying (52). Then

1. Let $1 \leq q<2$ and $w_{0} \in L^{q} \cap L_{\sigma}^{2}$., then every weak solution $w(t)$ with (52) satisfies

$$
\|w(t)\|_{2} \leq C(1+t)^{-\frac{n}{2}\left(\frac{1}{q}-\frac{1}{2}\right)} \text { as } t \rightarrow \infty
$$

2. Let $w_{0} \in L^{1} \cap L_{\sigma}^{2}$ and $\int|y|\left|u_{0}(y)\right| d y<\infty$. Given $\varepsilon>0$ arbitrarily small, there is a constant $\delta_{1}=\delta_{1}(\varepsilon) \in(0, \delta]$ such that if $\|V\| \leq \delta_{1}$, then every weak solution $w(t)$ with (52) satisfies

$$
\|w(t)\|_{2} \leq C(1+t)^{-\frac{n}{4}-\frac{1}{2}+\varepsilon} \text { as } t \rightarrow \infty
$$


The proof of the theorem combines the Fourier splitting method, with an analysis of large time behavior of solutions to the initial value problem for the linearized equations:

$$
\begin{aligned}
& \partial_{t} u-\Delta u+V \cdot \nabla u+u \cdot \nabla V+\nabla p=0 \\
& \operatorname{div} u=0 \\
& u(\cdot, s)=f
\end{aligned}
$$

This system is studied in $\mathbb{R}^{n} \times(s, \infty)$, where $s \geq 0$ is the given initial time, recall that $V$ is time-dependent and the system is non-autonomous. The energy $\|u(t)\|_{2}^{2}$ is split into time-dependent low and high frequency regions in the Fourier side. The decay rate is determined from the low frequency part and the argument is based on analyzing the integral equation in terms of the evolution operator $T(t, s)$ in $L_{\sigma}^{2}$ for all $t \geq s \geq 0$. That is the equation given by

$$
u(t)=T(t, s) u(s)-\int_{s}^{t} T(t, \tau) P(u \cdot \nabla u)(\tau) d \tau
$$

which provides a unique solution to (53). Technical difficulties prevent the use of standard techniques, but Yamazaki's idea of using real interpolation [207] is very useful in the study the operator $T(t, s)$.

\subsubsection{Two dimensional case. On flows with radial energy decomposition.}

The work by C. Bjorland and C. Niche [11] analyzes the behavior of infinite energy solutions $u$ satisfying

$$
\begin{array}{r}
u_{t}+u \cdot \nabla u+\nabla p-\Delta u=-u \cdot \nabla v-v \cdot \nabla u, \\
\nabla \cdot u=0, \quad u(0)=u_{0} \in L^{2}\left(\mathbb{R}^{2}\right)
\end{array}
$$

where $v$ is an a priori given vector field which satisfies

$$
\left\|\nabla^{\alpha} v\right\|_{L^{\eta}\left(\mathbb{R}^{2}\right)} \leq C t^{-\frac{1}{2}-\frac{\alpha}{2}+\frac{1}{\eta}}, \quad \text { where } \begin{cases}\alpha=0, & \text { if } 2<\eta<\infty \\ \alpha=0 \text { or } \alpha=1 & \text { if } \eta=\infty .\end{cases}
$$

System (55) can be obtained for example as the difference of two solutions to the NavierStokes equations: $u=w-v$. In [11] the the corresponding data for $w$ is decomposed as $w_{0}=u_{0}+v_{0}$, where $u_{0} \in L^{2}\left(\mathbb{R}^{2}\right)$ has finite energy while $v_{0}=v(\cdot, 0)$, with $v$ an a priori given solution of NS (possibly of infinite energy), that satisfies (56).

If $e^{t \Delta} u_{0}$ decays in the $L^{2}$-norm at an algebraic rate, then it is claimed in [11] that the solution $u=w-v$ of (55) remains in $L^{2}$ and decays at the same algebraic rate (if this rate is not too large). This process would allow to obtain the asymptotic behavior of solutions with infinite energy. However, the proof main result of that paper seems to be built upon an incorrect Gronwall inequality [11, p.674]. This erratum was pointed to the authors of the present review paper by an anonymous referee. As a result, the main theorem of [11] 
does not seem to be proved successfully any longer. We therefore reformulate it as an interesting open problem:

Let $u$ be a global solution to (55), $v$ satisfy (56), and assume:

(i) $\forall t_{0}>0, \exists C_{t_{0}}>0$, such that $\forall T>t_{0} \sup _{t_{0} \leq t \leq T}\|u(t)\|_{2} \leq C_{t_{o}}(1+T)^{\frac{1}{2}}$.

(ii) For some $\gamma \in[0,1],\left\|e^{\Delta t} u_{0}\right\|_{2}^{2} \leq C(1+t)^{-\gamma}$.

Can one deduce from the above that $\forall t \geq t_{0},\|u(t)\|_{2} \leq \tilde{C}_{t_{o}}(1+t)^{-\gamma}$ ?

Assumption (i) is satisfied in many natural situations. If positively answered, the above question would have several consequences, including an improved decay for solutions arising from data in $w_{0}=v_{0}+u_{0} \in \dot{B}_{r}^{\frac{2}{r}-1, q}\left(\mathbb{R}^{2}\right)$, or arising from data with radial energy decomposition. See [11] for more a detailed discussion.

Remark 4.5. An anonymous referee pointed out to us, that a partial solution of the open problem can be found in the paper by Y. Maekawa [151]. In [151] the vector field $v(t)$ is assumed to be a Kozono-Yamazaki solution of the Navier-Stokes equations with data $v_{0} \in L^{2, \infty}(\Omega)$ and $\left\|v_{0}\right\|_{L^{2, \infty}(\Omega)} \ll 1$, where $\Omega=R^{2}$ or is an exterior domain. Such solutions satisfy

$$
\sup _{t>0}\|v(t)\|_{L^{2, \infty}(\Omega)}+\sup _{t>0} t^{\frac{1}{4}}\|v(t)\|_{L^{4}(\Omega)} \ll 1
$$

The results obtained in [151] analyze the stability of the solutions. The main result obtained is the following:

Theorem 4.6. There exists a constant $\delta>0$ such that for any $u_{0} \in L_{\sigma}^{2, \infty}(\Omega)+L^{2}(\Omega)_{\sigma}^{\|\cdot\|_{L^{2}, \infty}}$ of the form

$$
u_{0}=v_{0}+w_{0}, \quad\left\|v_{0}\right\|_{L^{2, \infty}(\Omega)} \leq \delta, \quad w_{0} \in L^{2}(\Omega)_{\sigma}^{\|\cdot\|_{L}^{2, \infty}},
$$

if $u(t)$ is the solution to the NS equations with data $u_{0}$, then the perturbation $w(t)=$ $u(t)-v(t)$ satisfies

$$
\lim _{t \rightarrow \infty}\|w(t)\|_{L^{2, \infty}(\Omega)}=0,
$$

and moreover

$$
\lim _{t \rightarrow \infty}\|w(t)\|_{L^{2}(\Omega)}=0
$$

\section{Other models}

\subsection{The MHD equations}

In plasma physics, the magnetohydrodynamics (MHD) equations describe the interactions between a magnetic field and a fluid made of moving electrically charged particles. In non- 
dimensional form, these equations can be written in the following way:

$$
\left\{\begin{array}{l}
\frac{\partial u}{\partial t}+(u \cdot \nabla) u-S(B \cdot \nabla) B+\nabla\left(p+\frac{S}{2}|B|^{2}\right)=\frac{1}{R_{e}} \Delta u \\
\frac{\partial B}{\partial t}+(u \cdot \nabla) B-(B \cdot \nabla) u=\frac{1}{R_{m}} \Delta B \\
\operatorname{div} u=\operatorname{div} B=0 \\
u(0)=u_{0} \quad \text { and } \quad B(0)=B_{0} .
\end{array}\right.
$$

Here the unknowns are the velocity field $u$ of the fluid, the pressure $p$ and the magnetic field $B$, all defined in $\mathbb{R}^{d}(d \geq 2)$. The constants $R_{e}>0$ and $R_{m}>0$ are respectively the Reynolds number and the magnetic Reynolds number; moreover $S=M^{2} /\left(R_{e} R_{m}\right)$, where $M$ is the Hartman number. From now on, unless otherwise stated, all these constants are assumed equal to 1 . After rescaling $u$ and $B$, it can be surmised that $S=R_{e}=1$.

Many of the results established for the Navier-Stokes equations have their analogue counterpart for the MHD equations. For example, M.E. Schonbek, T. Schonbek and E. Suli [187] established that, if $u_{0}$ and $B_{0}$ are in $L^{2}\left(\mathbb{R}^{3}\right)$ and satisfy suitable moment conditions, then weak solutions (at least those satisfying a strong energy inequality) satisfy

$$
c(1+t)^{-\frac{n+2}{4}} \leq\|u(t)\|_{2}+\|B(t)\|_{2} \leq C(1+t)^{-\frac{n+2}{4}},
$$

where $c \geq 0$, but $c$ is generically strictly positive. In fact, $c=0$ if and only if the matrices $\int_{0}^{\infty} \int(u \otimes u-B \times B)$ and $\int_{0}^{\infty} \int(u \otimes B-B \otimes u)$ are multiple of the identity matrix. Nontrivial solutions inside this class with faster dissipation rates can been constructed in even dimension by adapting the classical construction of Beltrami flows (see [187]) and in any dimension putting symmetry conditions, similar to those as mentioned in section 2.6.

R. Agapito and M.E. Schonbek [2] addressed the limit case of infinite magnetic Reynolds number, in which case there is no diffusion in the second equation in (MHD). Under the conditional assumption that there exists a global strong solution $(u, B)$, with magnetic field $B \in L^{\infty}\left(\mathbb{R}^{3} \times \mathbb{R}^{+}\right)$, then, as time t goes to infinity, $\|u(t)\|_{2} \rightarrow 0$ and $\|B(t)\|_{2}$ converges to a non-negative constant. This result shows that the diffusion in the velocity is sufficient to prevent compensatory oscillations between the two energies of the velocity and the magnetic field. Moreover, in the presence of both fluid and magnetic diffusion the energies of the velocity and the magnetic field decay to zero, but if the data are only assumed to be in $L^{2}$, there cannot be a uniform rate for the energy of the solutions.

S. Weng [204] derived sharp bounds for moments and higher-order derivatives similar to (28) (both for $u$ and $B$ ) using the approach of Kukavica and Torres, as mentioned in Section 2.9.2. His results holds true also for the Hall-MHD equation, extending earlier studies by D.Chae and M.E. Schonbek [48]. The Hall-MHD equations are obtained putting an additional $\nabla \times((\nabla \times B) \times B)$ term in the second equation in (MHD): this is the Hall term that takes into account the magnetic reconnection effects, and is important when the magnetic shear is large. 


\subsection{The Boussinesq system}

The Boussinesq system is a well established model for studying the heat transfer in incompressible viscous flows. The Boussinesq approximation consists in neglecting density variations in the continuity equation and the heating process of viscous dissipation: the temperature variations are taken into consideration through an additional buoyancy forcing term acting on the fluid, leading to the following Cauchy problem

$$
\left\{\begin{array}{l}
\partial_{t} \theta+u \cdot \nabla \theta=\kappa \Delta \theta \\
\partial_{t} u+u \cdot \nabla u+\nabla p=\nu \Delta u+\beta \theta e_{3} \\
\nabla \cdot u=0 \\
\left.u\right|_{t=0}=u_{0},\left.\quad \theta\right|_{t=0}=\theta_{0} .
\end{array} \quad x \in \mathbb{R}^{3}, t \in \mathbb{R}_{+}\right.
$$

Here $u: \mathbb{R}^{3} \times \mathbb{R}^{+} \rightarrow \mathbb{R}^{3}$ is the velocity field. The scalar fields $p: \mathbb{R}^{3} \times \mathbb{R}^{+} \rightarrow \mathbb{R}$ and $\theta: \mathbb{R}^{3} \times$ $\mathbb{R}^{+} \rightarrow \mathbb{R}$ denote respectively the pressure and the temperature of the fluid. Moreover, $e_{3}=(0,0,1)$, and $\beta \in \mathbb{R}$ is a physical constant related to the gravity. In what follows for simplicity it is assumed that all these constants are equal to 1.

To construct a global weak solution, it is only necessary to assume that $u_{0} \in L_{\sigma}^{2}\left(\mathbb{R}^{3}\right)$ and, e.g., that $\theta_{0} \in L^{2}\left(\mathbb{R}^{3}\right)$. See, e.g., R. Danchin and M. Paicu's paper [59], where such construction is performed in the more general setting $\kappa \geq 0$. Such solutions then satisfy the energy inequality

$$
\|u(t)\|_{2}^{2}+2 \int_{0}^{t}\|\nabla u(s)\|^{2} d s \leq C\left(\left\|u_{0}\right\|+t^{2}\left\|\theta_{0}\right\|^{2}\right)
$$

for all $t \geq 0$, and some absolute constant $C>0$. The above upper bound can be improved under the slightly more stringent but natural condition $\theta_{0} \in L^{1} \cap L^{2}\left(\mathbb{R}^{3}\right)$. In this case one easily gets the bound $\|u(t)\|_{2}^{2}=\mathcal{O}(\sqrt{t})$ as $t \rightarrow \infty$, and $\|\theta(t)\|_{2}^{2}=\mathcal{O}\left(t^{-3 / 2}\right)$. The latter bound looks optimal, as it agrees with that of the solution to linear heat equation $e^{t \Delta} \theta_{0}$, but the bound of the velocity is physically strange, as one would not a priori expect that the energy can grow arbitrarily large. However, L. Brandolese and M.E. Schonbek [26] proved that, for strong solutions arising from small and well localized data, as the matter of fact $\|u(t)\|_{2}^{2} \sim \sqrt{t}$ as $t \rightarrow \infty$, provided that the mean $\int \theta_{0}$ of the initial temperature is nonzero. When $\int \theta_{0}=0$ the energy goes to zero, in the large time, accordingly to what happens in the Navier-Stokes case $\theta \equiv 0$.

The method of [26] consists in obtaining point-wise asymptotic profiles for the velocity in the parabolic region $|x| \gg \sqrt{t}$. Namely, if

$$
\left\|\theta_{0}\right\|_{1}<\epsilon, \quad \text { ess } \sup _{x \in \mathbb{R}^{3}}|x|^{3}\left|\theta_{0}(x)\right|<\epsilon, \quad \text { ess } \sup _{x \in \mathbb{R}^{3}}|x|\left|u_{0}(x)\right|<\epsilon
$$

and $\epsilon>0$ is small enough, then one can construct a unique global solution to (57) satisfying suitable point-wise estimate. If in addition $\left|u_{0}(x)\right| \leq C(1+|x|)^{-a}$ and $\left|\theta_{0}(x)\right| \leq C(1+|x|)^{-b}$ for some $a>3 / 2$ and $b>3$, then

$$
u(x, t)=e^{t \Delta} u_{0}(x)+\left(\int \theta_{0}\right) t\left(\nabla E_{x_{3}}\right)(x)+\mathcal{R}(x, t)
$$


where $\mathcal{R}(x, t)$ is a lower order term with respect to $t \nabla E_{x_{3}}(x)$ for $|x| \gg \sqrt{t}$, namely, $\lim _{\frac{|x|}{\sqrt{t}} \rightarrow \infty} \frac{\mathcal{R}(x, t)}{t|x|^{3}}=0$. Such point-wise asymptotic profiles can be used to deduce upper and lower bound estimates for weighted $L^{p}$-norms:

$$
c(1+t)^{\frac{1}{2}\left(r+\frac{3}{p}-1\right)} \leq\left\|(1+|x|)^{r} u(t)\right\|_{p} \leq C(1+t)^{\frac{1}{2}\left(r+\frac{3}{p}-1\right)},
$$

for all $r \geq 0,1<p<\infty$ and $r+\frac{3}{p}<3$. The constant $c$ in the above lower bound is strictly positive if and only if the mean of $\int \theta_{0} \neq 0$. The case $r=0$ and $p=0$ agrees with the energy growth phenomenon $\|u(t)\|_{2}^{2} \sim \sqrt{t}$ mentioned before.

It is interesting to observe that this result is specific to the whole space. Indeed, when studying the system (57) in the half space $\mathbb{R}_{+}^{3}$ (complementing it with the boundary conditions $u=\theta=0$ on $\partial \mathbb{R}_{+}^{3} \times(0, \infty)$, then the energy growth estimate (61) is no longer true. In fact, P. Han and M.E. Schonbek [99] showed that $\|u(t)\|_{L^{2}\left(\mathbb{R}_{+}^{3}\right)} \rightarrow 0$ even if no cancellation of the temperature holds. The only conditions to achieve this decay to zero of the energy of a weak solution are suitable size and decay conditions of the initial data, namely $u_{0} \in L_{\sigma}^{2}\left(\mathbb{R}_{+}^{3}\right), \theta_{0} \in L^{1} \cap L^{2}\left(\mathbb{R}_{+}^{3}\right), \int_{\mathbb{R}_{+}^{3}} x_{3}\left|\theta_{0}\right|<\infty$ and $\left\|\theta_{0}\right\|_{1}$ small enough. In the exterior domain, to prevent energy growth, P. Han [98] put the decay assumption $\left\|e^{t \Delta} \theta_{0}\right\|_{1} \leq C(1+t)^{-\frac{1}{2}}$, that however is not generic and requires cancellations. Under additional assumptions, decay of higher order derivative can be obtained: see [99], [203].

One can view the energy growth result included estimates (61) as a physical limitation of the model (57) when studying heat transfers in unbounded domains for long time intervals. The correction proposed in [67] consists in replacing the constant vector $\beta e_{3}$ with a term $\nabla G(x)$ where $G$ is a harmonic function behaving like $1 /|x|$ as $|x| \rightarrow \infty$, accordingly to Newton's gravitational law. More precisely in the model used in [67] the self-gravitation of the fluid is neglected, hence the origin of the gravitational force must be an object placed outside the fluid domain $\Omega$ and hence

$$
G(x)=\int_{\mathbb{R}^{3}} \frac{1}{|x-y|} m(y) \mathrm{d} y, \text { with } m \geq 0, \operatorname{supp}[m] \subset \mathbb{R}^{3} \backslash \Omega,
$$

where $m$ denotes the mass density of the object acting on the fluid by means of gravitation. It follows that the forcing term $G$ is a harmonic function in $\Omega$, and as stated above $G(x) \approx$ $1 /|x|$ as $|x| \rightarrow \infty$. Motivated by the previous observations, this Boussinesq system (usually referred to as the Oberbeck-Boussinesq system ) is considered in a domain $\Omega=R^{3} \backslash K$, exterior to a compact set $K$. The correction introduced by the forcing term $\mathrm{G}$ prevents the energy to become arbitrarily large. Furthermore when the solution is considered with zero boundary condition on $\partial K$ and initial conditions $u_{0} \in L_{\sigma}^{2}, \theta \in L^{1} \cap L^{\infty}$. Then $u(t) \rightarrow 0 \in L^{2}$ and $\theta \rightarrow 0 \in L^{p}, 1<p \leq \infty$.

\subsection{Nematic liquid crystals}

The flow of nematic liquid crystals can be treated as slow moving particles where the fluid velocity and the alignment of the particles influence each other. The hydrodynamic theory of liquid crystals was established by Ericksen $[62,63]$ and Leslie $[146,147]$ in the 
1960's. As F.M. Leslie points out in his 1968 paper: "liquid crystals are states of matter which are capable of flow, and in which the molecular arrangements give rise to a preferred direction". The discussion is first centered on the asymptotics of two simplified models defined in $\Omega \times(0, T)$, where $\Omega$ is either a bounded domain of $\mathbb{R}^{n}$ or $\Omega=\mathbb{R}^{n}$, with $n=2$ or $n=3$. The models are given by

$$
\begin{array}{r}
u_{t}+u \cdot \nabla u+\nabla \pi=\nu \Delta u-\nabla \cdot(\nabla d \otimes \nabla d), \\
d_{t}+u \cdot \nabla d=\Delta d-\mathcal{H}_{i}(d), \\
\nabla \cdot u=0 .
\end{array}
$$

Here, $\pi=\pi(x, t)$ and $u=u(x, t)$ are the fluid pressure and velocity, and $d=d(x, t)$ is the director field representing the alignment of the molecules. The constant $\nu>0$ is viscosity coefficient. The terms $\mathcal{H}_{i}, i=1,2$ will determine which system is used. They are chosen to be

$$
\mathcal{H}_{1}=f(d)=\nabla F(d)=\frac{1}{\eta^{2}}\left(|d|^{2}-1\right) d, \text { or } \mathcal{H}_{2}=\nabla d|\nabla d|^{2}
$$

Depending on the choice of $\mathcal{H}_{i}, i=1,2$ the systems will be referred as (62) by LCD1 or LCD2 respectively. For LCD1 the forcing term $\nabla d \otimes \nabla d$ is the stress tensor of the energy about the director field $d$, with energy of the form:

$$
\frac{1}{2} \int_{\mathbb{R}^{3}}|\nabla d|^{2} d x+\int_{\mathbb{R}^{3}} F(d) d x, \text { where } F(d)=\frac{1}{4 \eta^{2}}\left(|d|^{2}-1\right)^{2},
$$

and $\eta$ a constant.

Remark 5.1. The $F(d)$ is the penalty term of the Ginzburg-Landau approximation of the original free energy for the director field with unit length. When $\eta \rightarrow 0$, i.e the penalty term disappears and, the term $\nabla d|\nabla d|^{2}$ should be recovered

There is a vast literature on the general behavior of liquid crystal systems. For background the reader is referred to $[56,108]$ and the references within. The asymptotic behavior of regular solutions to the flow of nematic liquid crystals was studied for bounded domains in $[148,206]$. In [206] Hao Wu shows that, with suitable initial conditions, the velocity converges to zero and the direction field converges to the steady solution of

$$
\left\{\begin{array}{l}
-\Delta d_{\infty}+f\left(d_{\infty}\right)=0, x \in \Omega \\
d_{\infty}(x)=d_{0}(x), x \in \partial \Omega
\end{array}\right.
$$

In [206] a Łojasiewicz-Simon [193] type inequality is used to derive the convergence when $\Omega$ is a bounded domain, with data satisfying $u_{0} \in H_{0}^{1}(\Omega), \nabla \cdot u_{0}=0, d_{0} \in H^{2}(\Omega)$. The following additional conditions are stipulated

- If $\Omega \subset \mathbb{R}^{2}$ suppose $\lim _{t \rightarrow \infty}\left(\|u(t)\|_{H^{1}}+\left\|d(t)-d_{\infty}\right\|_{H^{2}}\right)=0$.

- If $\Omega \subset \mathbb{R}^{3}$ suppose the viscosity constant is sufficiently large. 
Then the unique global solution satisfies

$$
\|u(t)\|_{H^{1}}+\left\|d(t)-d_{\infty}\right\|_{H^{2}} \leq(1+t)^{-\frac{\theta}{1-2 \theta}}, \forall t \geq 0 .
$$

Here $\theta \in\left(0, \frac{1}{2}\right)$ is the constant from the Lojasiewicz-Simon type inequality.

Lack of compactness considerations do not allow to use similar arguments in the whole space $\mathbb{R}^{3}$ and, other methods need to be used. In [56] Dai, Qing and M. Schonbek establish a non-optimal decay rate for regular solutions in $\mathbb{R}^{3}$ to LCD1, with small initial data. Moreover the initial director field is required to tend to a constant unit vector $w_{0}$, as the space variable tends to infinity:

$$
\lim _{|x| \rightarrow \infty} d_{0}(x)=w_{0}
$$

This behavior at infinity of the initial director field allows to obtain the stability without needing the Liapunov reduction and Łojasiewicz-Simon inequality. Note that $w_{0}$ is the simplest case of a steady solution to (63).

The decay estimates in [56] were improved in [58] by Dai and M. Schonbek, were optimal rates in $H^{m}$ were obtained in the sense that they coincide with the rates of the underlying linear equations. The following initial conditions were supposed

$$
\begin{gathered}
u(x, 0)=u_{0}(x), \quad \nabla \cdot u_{0}=0, \quad d(x, 0)=d_{0}(x), \quad\left|d_{0}(x)\right|=1, \\
u_{0} \in H^{m}\left(\mathbb{R}^{3}\right) \cap L^{1}\left(\mathbb{R}^{3}\right), \quad d_{0}-w_{0} \in H^{m+1}\left(\mathbb{R}^{3}\right) \cap L^{1}\left(\mathbb{R}^{3}\right)
\end{gathered}
$$

for any integer $m \geq 1$ with a fixed vector $w_{0} \in S^{2}$, that is, $\left|w_{0}\right|=1$.

Theorem 5.2. [58] Assume the initial data $\left(u_{0}, d_{0}\right)$ satisfies the conditions above, Let $(u, \pi, d)$ be the regular solution. There exists a small number $\epsilon_{0}>0$ such that if $\left\|u_{0}\right\|_{H^{1}\left(\mathbb{R}^{3}\right)}^{2}$ $\left\|d_{0}-w_{0}\right\|_{H^{2}\left(\mathbb{R}^{3}\right)}^{2} \leq \epsilon_{0}$, then, for all $m \geq 0$ and $2 \leq p \leq \infty$

$$
\left\|D^{m}\left(d(\cdot, t)-w_{0}\right)\right\|_{p}+\left\|D^{m} u(\cdot, t)\right\|_{p} \leq C_{m}(1+t)^{-\left(\frac{3}{2}\left(1-\frac{1}{p}\right)+\frac{m}{2}\right)},
$$

The constant $C_{m}$ depends on initial data, $\nu, \eta$ and $m$.

The proof of Theorem 5.2 first established decay for the director vector and then uses this decay to show that the forcing term " $\nabla \cdot(\nabla d \otimes \nabla d)$ " decays sufficiently fast to be able to implement a modified Fourier splitting argument.

The decay for solutions to the system LCD2, the system without the Ginzburg-Landau penalty term, was obtained by Shengquan Liu and Xinying Xu in [150]. Specifically the results in [150] deal with the decay of smooth solutions with data $u_{0}$ satisfying

$$
u_{0} \in H^{m}, d_{0} \in H^{m+1}, m \geq 3, \text { and }\left\|u_{0}\right\|_{2}+\|\nabla d\|_{2} \leq \epsilon, \operatorname{div} u_{0}=0 .
$$

If $m \geq 3$, solutions with data satisfying the last conditions are shown to decay at the rate:

$$
\left\|\nabla^{l} u\right\|_{2}^{2}+\left\|\nabla^{l} \nabla d\right\|_{2}^{2} \leq C(t+1)^{-\frac{3+2 l}{2}}, l=1,2
$$


These rates are optimal for the velocity, but not for the director vector. The work in [150] was improved by Gao et al. [89], where for $m \geq 1$ and, under slightly stronger hypothesis, optimal decay rates where obtained for both the velocity and the director vector. Specifically in [89] the data is supposed to satisfy $\left(u_{0}, d_{0}-w_{0}\right) \in H^{m} \times H^{m+1}, m \geq$ 1 , div $u_{0}=0$ and $\left\|u_{0}\right\|_{H^{1}}+\left\|\nabla\left(d-w_{0}\right)\right\|_{H^{2}} \leq \epsilon$, where $w_{0}$ is a fixed constant unit vector. The methods used in both papers are similar, both used Fourier splitting and involved energy estimates. In particular clever energy estimates are introduced to handle the nonlinear term $|\nabla d|^{2} \nabla d$.

For general information on the system LCD2 for bounded domains the reader is referred to M. Hieber et al. [108]. As stated by the authors [108]: by means of the associated energy functional, [they] prove convergence of a solution to an equilibrium, whenever the solution is eventually bounded in the natural state space.

\section{$Q$-tensor model}

A frequently used hydrodynamic model of nematic liquid crystals has the local configuration of the crystals represented by a $\mathbb{Q}$-tensor $=\mathbb{Q}(t, x) \in \mathbb{R}_{\text {sym }, 0}^{3 \times 3}$, a symmetric traceless matrix, and the motion described through an Eulerian velocity field $u=u(t, x)$. Here $(t, x) \in \mathbb{R}_{+} \times \mathbb{R}^{3}$ and, the system has the form

$$
\begin{aligned}
\partial_{t} \mathbb{Q}+\operatorname{div}(\mathbb{Q} u)-\mathcal{S}(\nabla u, \mathbb{Q}) & =\Delta \mathbb{Q}-\mathcal{L}[\partial F(\mathbb{Q})], \\
\partial_{t} u+\operatorname{div}(u \otimes u)+\nabla p & =\Delta u+\operatorname{div} \Sigma(\mathbb{Q}) \\
\operatorname{div} u & =0 .
\end{aligned}
$$

The operator $\mathcal{L}$ denotes the projection onto the space of traceless matrices, and $F$ is a special potential function. The tensors $\mathcal{S}$ and $\Sigma$ both depend nonlinearly on the tensor $\mathbb{Q}$. In [55], Dai, Feireisl, Rocca, Schimperna and M. Schonbek investigate the existence and decay of solutions to (66) for data satisfying

$u_{0} \in L^{1} \cap L^{2}\left(\mathbb{R}^{3} ; \mathbb{R}^{3}\right), \operatorname{div} u_{0}=0, Q_{0} \in L^{1} \cap W^{1,2}\left(\mathbb{R}^{3} ; \mathbb{R}_{\text {sym }, 0}^{3 \times 3}\right),\left|\mathbb{Q}_{0}(x)\right| \leq r_{2}$ for a.e. $x \in \mathbb{R}^{3}$,

For such solutions, it is shown, under appropriate conditions on the potential function $F$ that

$$
\|u(t, \cdot)\|_{L^{2}\left(\mathbb{R}^{3} ; \mathbb{R}^{3}\right)}+\|\mathbb{Q}(t, \cdot)\|_{W^{1,2}\left(\mathbb{R}^{3} ; \mathbb{R}^{3 \times 3}\right)} \leq c(1+t)^{-\alpha}
$$

Where $\alpha=\frac{15}{28}$. This decay rate can be improved to $\frac{3}{4}$ under the additional condition

$$
F(\mathbb{Q}) \geq \lambda|\mathbb{Q}|^{2} \text { in } B_{r_{1}} \text {, some } \lambda>0, \text { and } B_{r_{1}}=\text { a ball of radius } r_{1}>0 \text {. }
$$

The decay rates in [55] are obtained by some quite technical modifications of the Fourier splitting technique.

For more information on related issues of nematic liquid crystals the reader is referred to [109], an dthe contributions in this handbook by M. Hieber and J. Saal [110]. 


\subsection{Quasi-Geostrophic sytem}

\section{Decay in the whole plane case}

Besides its intrinsic mathematical interest, the dissipative 2D Quasi-Geostrophic equation describes phenomena arising in meteorology and oceanography. The model can be derived from the General Quasi Geostrophic equations by assuming constant potential vorticity and constant buoyancy frequency. See Constantin et al. [52] and Pedlosky [175]. The equations are given by

$$
\begin{array}{r}
\theta_{t}+(u \cdot \nabla) \theta+(-\Delta)^{\alpha} \theta=0 \\
\theta(x, 0)=\theta_{0}(x)
\end{array}
$$

here $\alpha \in(0,1], t>0$ and, $x \in \Omega$, with $\Omega=\mathbb{R}^{2}$ or $\Omega$ is an exterior domain of the form $\Omega=\mathbb{R}^{2} \backslash \overline{\mathcal{O}}$, with $\mathcal{O}$ an open connected bounded set in $\mathbb{R}^{2}$. The variable $\theta=\theta(x, t)$ is a real scalar function, standing for the temperature of the fluid, $u$ is the velocity field, determined by the scalar function $\psi$ (the stream function) through

$$
u=\left(u_{1}, u_{2}\right)=\left(-\frac{\partial \psi}{\partial x_{2}}, \frac{\partial \psi}{\partial x_{1}}\right), \quad \Lambda \psi=-\theta, \quad \text { where } \Lambda=(-\Delta)^{\frac{1}{2}}
$$

Decay in the whole space $\mathbb{R}^{2}$. Constantin and $\mathrm{Wu}[53]$ showed that, for datum $\theta_{0}$ in $L^{2} \cap L^{1}$, the corresponding solution solution of (67) satisfies

$$
\|\theta(t)\|_{2} \leq C(1+t)^{-\frac{1}{2 \alpha}}, \quad t \geq 0
$$

where $C$ depends only on the $L^{2}$ and $L^{1}$ the data.

Their proof relies on a modified version of the Fourier splitting method developed in $[188,189]$, The second main tool used was the retarded mollifiers method of Caffarelli, Kohn and Nirenberg [29]. The authors of [53] proved that for generic initial data, the decay rate (68) is optimal.

Remark 5.3. The results in [53] also work with $\Omega=\mathbb{T}^{2}$ a two dimensional torus.

Remark 5.4. The kind of modified FS used in [53], was also employed by Linghai Zhang in [209] to get optimal decay in 2 dimensions for solutions to the Navier-Stokes.

The results in [53] were extended by M. Schonbek and T. Schonbek in [189] to obtain the decay of the derivatives: supposing that $\theta_{0} \in L^{1}\left(\mathbb{R}^{2}\right) \cap H^{m}\left(\mathbb{R}^{2}\right)$, then

$$
\left\|\Lambda^{\beta} \theta(t)\right\|_{2} \leq C(t+1)^{-\frac{\beta+1}{2 \alpha}},
$$

where the constant depends on ly on the $L^{1}$ and $H^{m}$ norms of the data.

Furthermore if the Riesz potential of the data $I_{\beta} \theta_{0}=\frac{\widehat{\theta}_{0}(\xi)}{|\xi|^{\beta}} \in L^{1}\left(\mathbb{R}^{2}\right)$, it is shown in [189], that the $L^{p}\left(\mathbb{R}^{2}\right)$ of the solutions decay at a rate that depends on the order of the derivatives, $\alpha$ and $\beta$. Moreover for data with Riesz potentials in $L^{1}\left(\mathbb{R}^{2}\right)$ it was shown that there is a slightly better $L^{2}$ decay rate towards $A \partial^{\gamma} \Lambda^{\beta} G_{\alpha}(t)$, where $A=\int_{\mathbb{R}^{2}}\left(I_{\beta} \theta_{0}\right)(x) d x$ and $G_{\alpha}(t) \theta_{0}=e^{-t \Lambda^{2 \alpha}} \theta_{0}$. 
Using rather general point-wise estimates for the fractional derivative $\Lambda^{\alpha} \theta$ and a positivity lemma, Córdoba and Córdoba [54] gave a proof of a maximum-principle and used it to establish decay of solutions when $\theta_{0}$ is in $L^{1} \cap L^{p}, 1<p<\infty$. More specifically, they showed that

$$
\|\theta(t)\|_{p} \leq C_{1}\left(1+C_{2} t\right)^{-\frac{p-1}{\alpha p}}, \quad t \geq 0
$$

where $C_{1}$ and $C_{2}$ are explicit constants. Working along the same lines, Ju [117] obtained an improved maximum principle, for $\theta_{0}$ in $L^{2} \cap L^{p}$, with $p \geq 2$ and a constant $C \neq 1$, of the form

$$
\|\theta(t)\|_{p} \leq\left\|\theta_{0}\right\|_{p}\left(1+\frac{C^{\frac{1}{p-2}}}{p-2} t\right)^{\frac{2-p}{2 p \alpha}} .
$$

When $p>2$, this last inequality also gives decay. Note that for $p=2$, i.e. $\theta_{0}$ in $L^{2}$, this expression reduces to $\|\theta(t)\|_{2} \leq\left\|\theta_{0}\right\|_{2}$.

In [188], M. Schonbek and T. Schonbek established rates of decay are for moments of the solutions to the QG equations, and also obtain lower bounds on decay rates of the solutions. For data in $L^{p} \cap L^{2}, p \in[1,2)$ algebraic decay can be found in [168] by Niche and Schonbek . Using ideas from Kato algebraic decay for large in $L^{q}$ norms with large $q$ are obtained in [168]. That is provided $\theta_{0} \in L^{\frac{2}{2 \alpha-1}}$ then the solutions and first derivatives decay in $L^{q}$ for $q \in\left[\frac{2}{2 \alpha-1}, \infty\right)$.

In the work of Carrillo and Ferreira [42-44], the asymptotics of the solutions to the 2DQG equations are carefully analyzed. In [44] it is shown that the solutions converge to an Oseen vortex-like solution in $L^{1}\left(\mathbb{R}^{2}\right)$, for initial datum with appropriate decay at infinity. In [43] stability is studied in $L^{p}$ and weak $L^{p}$ spaces. The third paper [42] gives a fairly complete analysis of the asymptotic behavior in certain $L^{p}$ spaces. It is shown that solutions with datum in $L^{1}\left(\mathbb{R}^{2}\right) \cap L^{\frac{2}{2 \alpha-1}}\left(\mathbb{R}^{2}\right)$ converge as time goes to infinity to a particular self-similar solution normalized by the mass. The convergence is algebraic in $L^{q}, 1 \leq q \leq \infty$ and it is for derivatives all orders. Moreover it is also shown that if the data is in $L^{2 /(2 \alpha-1)}\left(\mathbb{R}^{2}\right)$, then the solutions and derivatives tend to zero in $L^{q}$, for $q \in\left[\frac{2}{2 \alpha-1}, \infty\right]$. The decay is non-uniform in $L^{2}$, and is algebraic for all the $L^{2}$ norms of the derivatives.

As stated in Section 2.12, the decay character technique [169] can be applied for the solutions and derivatives of (67). Here only the results are stated and the reader is referred to [169] for more details.

Theorem 5.5 ([169]). Let $\theta_{0} \in L^{2}\left(\mathbb{R}^{2}\right), \alpha \in(0,1]$ with decay character $r^{*}=r^{*}\left(\theta_{0}\right)$, then there exists constants $C_{i}>0, i=1, \cdots, 5$ so that

$$
\begin{aligned}
& \text { - If } r^{*} \leq 1-\alpha \Rightarrow C_{1}(1+t)^{-\frac{1}{\alpha}\left(1+r^{*}\right)} \leq\|\theta(t)\|_{2}^{2} \leq C_{2}(1+t)^{-\frac{1}{\alpha}\left(1+r^{*}\right)} . \\
& \text { - If } r^{*} \in(1-\alpha)[1,2], r^{*} \leq 1 \Rightarrow C_{3}(1+t)^{-\frac{1}{\alpha}\left(1+r^{*}\right)} \leq\|\theta(t)\|_{2}^{2} \leq C_{4}(1+t)^{-\frac{1}{\alpha}(2-\alpha)} . \\
& \text { - If } r^{*}>1, r^{*} \geq 2(1-\alpha) \Rightarrow\|\theta(t)\|_{2}^{2} \leq C_{5}(1+t)^{-\frac{1}{\alpha}(2-\alpha)} .
\end{aligned}
$$


(ii) If $\theta_{0} \in H^{s}\left(\mathbb{R}^{2}\right), \frac{1}{2}<\alpha \leq 1$. For $r_{s}^{*}=r_{s}^{*}\left(\theta_{0}\right)$ there exists constants $C$ such that

$$
\begin{aligned}
& \text { - If } r_{s}^{*} \leq 1-\alpha \Rightarrow\|\theta(t)\|_{\dot{H}^{s}}^{2} \leq C(t+1)^{-\frac{1}{\alpha}\left(s+1+r^{*}\right)} ; \\
& \text { - If } r_{s}^{*} \geq 1-\alpha \Rightarrow\|\theta(t)\|_{\dot{H}^{s}}^{2} \leq C(t+1)^{-\frac{1}{\alpha}(s+2-\alpha)} .
\end{aligned}
$$

\section{Decay in exterior domains}

Decay in exterior domains for the 2DQG equations is studied in the papers by T. Schonbek and L. Kosloff $[127,128]$. The exterior domains considered are as follows: $\Omega=\mathbb{R}^{n} \backslash \mathcal{O}$ where $\mathcal{O}$ is a non-empty open, bounded, and connected subset of $\mathbb{R}^{n}$. For the particular case of the 2 DQG equations " $n$ " is taken to be 2 .

In [127] the authors first generalize the Fourier transform introduced by Ramm [177] in 3 dimensions to $\mathrm{n}$ dimensions with $n \geq 2$. This allows to obtain spectral representations of the Laplacian and fractional Laplacian in exterior domains and, moreover opens new ways to investigate questions in such domains. In particular it is used in 2 dimensions to study the decay of solutions to the 2DQG equations. In [127] the QG equations (67) are considered in the critical case when $\alpha=\frac{1}{2}$ for solutions $\theta \in C\left([0, \infty), H^{2}(\Omega)\right)$ with data in $L^{1}(\Omega) \cap L^{2}(\Omega)$. In [128], the decay was extended to weak solutions with $\alpha \in(0,1]$. The rates obtained were optimal, as they coincide with the rates of solutions to the underlying linear equations:

$$
\|\theta(t)\|_{2}^{2} \leq C(t+1)^{-\frac{1}{\alpha}}
$$

with $C$ depending only on $\left\|\theta_{0}\right\|_{L^{1} \cap L^{2}}$. In summary the work in $[127,128]$ combines three techniques:

- A Fourier transform which can be used for exterior domains and gives a spectral representation for the Laplacian and the fractional Laplacian.

- A localization of the operator $\Lambda,[30,198]$.

- An interesting and quite involved modification to the Fourier splitting technique, using the spectral decomposition via the Fourier transform for exterior domains.

\subsection{Incompressible inhomogeneous NS}

The motion of incompressible flows of mixing fluids with different densities, or of fluid flows with melted substances inside it, can be modeled by the following system

$$
\left\{\begin{array}{l}
\partial_{t} \rho+\nabla \cdot(\rho u)=0 \\
\partial_{t}(\rho u)+\nabla \cdot(\rho u \otimes u)-\nabla \cdot(\mu \mathcal{M})+\nabla \Pi=0, \quad(x, t) \in \mathbb{R}^{3} \times \mathbb{R}^{+}, \\
\nabla \cdot u=0 \\
\left.\rho\right|_{t=0}=\rho_{0},\left.\quad u\right|_{t=0}=u_{0},
\end{array}\right.
$$


where the scalar functions $\rho=\rho(x, t)$ and $\Pi=\Pi(x, t)$ are respectively the fluid density and the pressure, and $u$ denotes, as usual, the velocity field. Moreover, $\mathcal{M}=\frac{1}{2}\left(\partial_{i} u_{j}+\partial_{j} u_{i}\right)$, and $\mu=\mu(\rho)$ is a smooth positive function on $[0, \infty)$, expressing the viscosity coefficient. The existence of global weak solutions, in any space dimension, is due to DiPerna and Lions, see [149], but their uniqueness is not known, even in the 2D case.

One often reformulates system (70) by introducing the modified density $a=-1+\frac{1}{\rho}$, assuming that $\rho>0$, i.e. that there is no vacuum. It is then possible to prove local or global well-posedness results, putting some smalless assumption on $u_{0}$ and $a_{0}=\left.a\right|_{t=0}$. The early results in this direction by A.V. Kažihov, O. Ladjženskaja and Solonnikov, have been considerably improved and extended in the more recent works by H. Abidi, R. Danchin, G. Gui, M. Paicu, P. Zhang, and others (see [1] and the references therein). In [1], Abidi, Gui and, Zhang, addressed the case of flows with viscosity independent on the density, i.e., $\mu(\rho)=$ constant $>0$. They proved that any a priori given global solution (in suitable Besov spaces) must decay as $t \rightarrow \infty:\|u(t)\|_{L^{2}}$ and $\|\nabla u(t)\|_{L^{2}}$ decay at the same rate as the solution to the heat equation, at least when the latter has an algebraic decay not exceeding a critical rate.

In the two-dimensional case, H. Huang and M. Paicu [115] got similar decay results, namely,

$$
\|u(t)\|_{L^{2}}^{2} \leq C(1+t)^{-\frac{1}{2}\left(\frac{2}{p}-1\right)}, \quad\|\nabla u(t)\|_{L^{2}}^{2} \leq C(1+t)^{-1-\frac{1}{2}\left(\frac{2}{p}-1\right)+\epsilon}
$$

for Lions' weak solution arising from $u_{0} \in L^{p}\left(\mathbb{R}^{2}\right) \cap H^{1}\left(\mathbb{R}^{2}\right), \rho_{0}-1 \in L^{2} \cap L^{\infty}\left(\mathbb{R}^{2}\right)$, in the case of a non-constant viscosity. The main technical restriction in [115] is that the viscosity coeffient must remain close to a positive constant. See also the very recent paper by J.-Y. Chemin and P. Zhang [47], for an original construction of a class of "large", yet global smooth solutions to the system (70) and the corresponding decay results.

\section{Conclusion}

The work in this chapter tries to summarize the main progress on decay of solutions to diffusive equations. starting from the years after Leray's 1934 pioneering paper on solution to the Navier-Stokes equations.

The results presented analyze the decay of solutions to the Navier-Stpkes, MHD, QG, Boussinesq and liquid crystal systems. In particular showing that under appropriate initial conditions the solutions decay at optimal rates, in the sense that their rates are the same as for their underlying linear counterpart. It is noted that for most of these systems if the solutions to the underlying linear equations decay to zero, so do the solutions to the corresponding nonlinear system. One exception being the solutions to the Boussinesq system in the whole space, in the case that the temperature has non zero initial mass. It is interesting to note that this is not so in the case of the half space, provided the data is small. A question of interest is then, if for large data in the half space the solution will still decay to zero. 
The models other than the Navier-Stokes, considered within the chapter had zero external forces. If forces are added which decay in $L^{p}$ or Sobolev spaces sufficiently rapidly then the methods used for systems with zero forces can be easily extended. A natural query then is how do the solutions to the diffusive systems behave in presence of slowly decaying forces.

Finally a very interesting problem is to analyze the decay of solutions to the MHD with no magnetic diffusion. As pointed out in the chapter there are some results provided specific bounds are supposed on the solutions.

In conclusion what is shown by the results in this chapter is that due to the diffusion, the nonlinearity has limited influence as time tends to infinity. The solutions tend to behave as their linear part when the decay rates of the latter are low.

\section{Cross references}

1. G. P. Galdi and J. Neustupa, Steady-state Navier-Stokes flow around a moving body, $[76]$.

2. I. Gallagher, em Critical function spaces for well-posedness of the Navier-Stokes initial value problems, [82].

3. T. Gallay and Y. Maekawa, Existence and Stability of Viscous Vortices, [84].

4. M. Hieber and J. Prss, Modeling and analysis of the Ericksen-Leslie equations for nematic liquid crystal flow, [110].

5. M. Hieber and J. Saal, The Stokes equation in the $L^{p}$-setting: Well-posedness and regularity properties, [111].

6. T. Hishida, Stationary Navier-Stokes flow in exterior domains and Landau solutions, [113].

7. H. Jia, V. Sverak and T.-P. Tsai, em Self-similar solutions to the non-stationary Navier-Stokes equations, [116].

8. M. Korobkov, K. Pileckas and R. Russo, Solution of Leray's Problem for Stationary Navier-Stokes Equations in Plane and Axially Symmetric Spatial Domains, [152].

\section{Acknowledgements}

The authors thank the anonymous reviewers for some very helpful suggestions and corrections which served to improve the presentation of this chapter. 


\section{References}

[1] H. Abidi, G. Gui, and P. Zhang, On the decay and stability of global solutions to the 3D inhomogeneous Navier-Stokes equations, Comm. Pure Appl. Math. 64 (2011), no. 6, 832-881.

[2] R. Agapito and M. Schonbek, Non-uniform decay of MHD equations with and without magnetic diffusion, Comm. Partial Differential Equations 32 (2007), no. 10-12, 1791-1812.

[3] C. Amrouche, V. Girault, M. E. Schonbek, and T. P. Schonbek, point-wise decay of solutions and of higher derivatives to Navier-Stokes equations, SIAM J. Math. Anal. 31 (2000), no. 4, 740-753.

[4] P. Auscher, S. Dubois, and P. Tchamitchian, On the stability of global solutions to Navier-Stokes equations in the space, J. Math. Pures Appl. (9) 83 (2004), no. 6, 673-697 (English, with English and French summaries).

[5] H.-O. Bae and L. Brandolese, On the effect of external forces on incompressible fluid motions at large distances, Ann. Univ. Ferrara Sez. VII Sci. Mat. 55 (2009), no. 2, 225-238.

[6] H.-O. Bae, L. Brandolese, and B. J. Jin, Asymptotic behavior for the Navier-Stokes equations with nonzero external forces, Nonlinear analysis $\mathbf{7 1}$ (2009), no. 12 (Electronique. Doi:10.1016/j.na.2008.10.074), e292-e302.

[7] H.-O. Bae and B. J. Jin, Temporal and spatial decays for the Navier-Stokes equations, Proc. Roy. Soc. Edinburgh Sect. A 135 (2005), no. 3, 461-477.

[8] H.-O. Bae and B. J Jin, Upper and lower bounds of temporal and spatial decays for the Navier-Stokes equations, J. Differential Equations 209 (2005), no. 2, 365-391.

[9] M. Ben-Artzi, Global solutions of two-dimensional Navier-Stokes and Euler equations, Arch. Rational Mech. Anal. 128 (1994), no. 4, 329-358.

[10] C. Bjorland, L. Brandolese, D. Iftimie, and M. Schonbek, $L^{p}$-solutions of the steady-state NavierStokes equations with rough external forces, Comm. Partial Differential Equations 36 (2011), no. 2, $216-246$.

[11] C. Bjorland and C. Niche, On the decay of infinite energy solutions to the Navier-Stokes equations in the plane, Phys. D 240 (2011), no. 7, 670-674.

[12] C. Bjorland and M. Schonbek, Existence and stability of steady-state solutions with finite energy for the Navier-Stokes equation in the whole space, Nonlinearity 22 (2009), no. 7, 1615-1637.

[13] Poincaré's inequality and diffusive evolution equations, Adv. Differential Equations 14 (2009), no. 3-4, 241-260. MR2493562 (2010a:35006)

[14] W. Borchers and T. Miyakawa, Algebraic $L^{2}$ decay for Navier-Stokes flows in exterior domains, Acta Math. 165 (1990), no. 3-4, 189-227.

[15] - Algebraic $L^{2}$ decay for Navier-Stokes flows in exterior domains. II, Hiroshima Math. J. 21 (1991), no. 3, 621-640. MR1148998 (93g:35111)

[16] _ $L^{2}$-decay for Navier-Stokes flows in unbounded domains, with application to exterior stationary flows, Arch. Rational Mech. Anal. 118 (1992), no. 3, 273-295.

[17] _ On stability of exterior stationary Navier-Stokes flows, Acta Math. 174 (1995), no. 2, 311382.

[18] L. Brandolese, On the localization of symmetric and asymmetric solutions of the Navier-Stokes equations in $\mathbb{R}^{n}$, C. R. Acad. Sci. Paris Sér. I Math. 332 (2001), no. 2, 125-130.

[19] Space-time decay of Navier-Stokes flows invariant under rotations, Math. Ann. 329 (2004), no. $4,685-706$.

[20] - Asymptotic behavior of the energy and point-wise estimates for solutions to the Navier-Stokes equations, Rev. Mat. Iberoamericana 20 (2004), no. 1, 223-256. 
[21] Application of the realization of homogeneous Sobolev spaces to Navier-Stokes, SIAM J. Math. Anal. 37 (2005), no. 2, 673-683 (electronic).

[22] Concentration-diffusion effects in viscous incompressible flows, Indiana Univ. Math. J. 58 (2009), no. 2, 789-806.

[23] _ Fine properties of self-similar solutions of the Navier-Stokes equations, Arch. Ration. Mech. Anal. 192 (2009), no. 3, 375-401.

[24] Characterization of solutions to dissipative systems with sharp algebraic decay, arXiv:1509.05928 (To appear on SIAM J. Math. Anal.)

[25] L. Brandolese and Y. Meyer, On the instantaneous spreading for the Navier-Stokes system in the whole space, ESAIM Control Optim. Calc. Var. 8 (2002), 273-285. A tribute to J. L. Lions.

[26] L. Brandolese and M. Schonbek, Large time decay and growth for solutions of a viscous Boussinesq system, Trans. Amer. Math. Soc. 364 (2012), no. 10, 5057-5090.

[27] L. Brandolese and F. Vigneron, New asymptotic profiles of nonstationary solutions of the NavierStokes system, J. Math. Pures Appl. (9) 88 (2007), no. 1, 64-86.

[28] - On the localization of the magnetic and the velocity fields in the equations of magnetohydrodynamics, Proc. Roy. Soc. Edinburgh Sect. A 137 (2007), no. 3, 475-495.

[29] L. Caffarelli, R. Kohn, and L. Nirenberg, Partial regularity of suitable weak solutions of the NavierStokes equations, Comm. Pure Appl. Math. 35 (1982), no. 6, 771-831.

[30] L. Caffarelli and L. Silvestre, An extension problem related to the fractional Laplacian, Comm. Partial Differential Equations 32 (2007), no. 7-9, 1245-1260.

[31] C. Calderón, Existence of weak solutions for the Navier-Stokes equations with initial data in $L^{p}$, Trans. Amer. Math. Soc. 318 (1990), no. 1, 179-200.

[32] M. Cannone, Ondelettes, paraproduits et Navier-Stokes, Diderot Editeur, Paris, 1995. With a preface by Yves Meyer.

[33] _ Harmonic analysis tools for solving the incompressible Navier-Stokes equations, Handbook of mathematical fluid dynamics. Vol. III. Edited by S.J. Friedlander and D. Serre, Elsevier (2004), 161-244.

[34] M. Cannone, C. He, and G. Karch, Slowly decaying solutions to incompressible Navier-Stokes system, Gakuto International Series. Mathematical Sciences and applications 35 (2011).

[35] M. Cannone and G. Karch, Smooth or singular solutions to the Navier-Stokes system?, J. Differential Equations 197 (2004), no. 2, 247-274.

[36] _ About the regularized Navier-Stokes equations, J. Math. Fluid Mech. 7 (2005), no. 1, 1-28.

[37] M. Cannone, Y. Meyer, and F. Planchon, Solutions auto-similaires des équations de Navier-Stokes, Séminaire sur les Équations aux Dérivées Partielles, 1993-1994, École Polytech., Palaiseau, 1994, pp. Exp. No. VIII, 12.

[38] M. Cannone and F. Planchon, Self-similar solutions for Navier-Stokes equations in $\mathbf{R}^{3}$, Comm. Partial Differential Equations 21 (1996), no. 1-2, 179-193.

[39] A. Carpio, Comportement asymptotique dans les équations de Navier-Stokes, C. R. Acad. Sci. Paris Sér. I Math. 319 (1994), no. 3, 223-228 (French, with English and French summaries). MR1288407 (95h:35174)

[40] - Asymptotic behavior for the vorticity equations in dimensions two and three, Comm. Partial Differential Equations 19 (1994), no. 5-6, 827-872.

[41] _ Large-time behavior in incompressible Navier-Stokes equations, SIAM J. Math. Anal. 27 (1996), no. 2, 449-475.

[42] J. Carrillo and L. Ferreira, The asymptotic behaviour of subcritical dissipative quasi-geostrophic equations, Nonlinearity 21 (2008), no. 5, 1001-1018. 
[43] _ Self-similar solutions and large time asymptotics for the dissipative quasi-geostrophic equation, Monatsh. Math. 151 (2007), no. 2, 111-142.

[44] Convergence towards self-similar asymptotic behavior for the dissipative quasi-geostrophic equations, Self-similar solutions of nonlinear PDE, Banach Center Publ., vol. 74, Polish Acad. Sci. Inst. Math., Warsaw, 2006, pp. 95-115.

[45] Th. Cazenave, F. Dickstein, and F. B. Weissler, Chaotic behavior of solutions of the Navier-Stokes system in $\mathbb{R}^{N}$, Adv. Differential Equations 10 (2005), no. 4, 361-398.

[46] Th. Cazenave and F. B. Weissler, Asymptotically self-similar global solutions of the nonlinear Schrödinger and heat equations, Math. Z. 228 (1998), no. 1, 83-120.

[47] J. Chemin and P. Zhang, Inhomogeneous incompressible Navier-Stokes Flows with slowly varying initial data, arXiv (2015).

[48] D. Chae and M. Schonbek, On the temporal decay for the Hall-magnetohydrodynamic equations, J. Differential Equations 255 (2013), no. 11, 3971-3982.

[49] J. Chemin and I. Gallagher, Wellposedness and stability results for the Navier-Stokes equations in $\mathbf{R}^{3}$, Ann. Inst. H. Poincaré Anal. Non Linéaire 26 (2009), no. 2, 599-624.

[50] Z. Chen, A sharp decay result on strong solutions of the Navier-Stokes equations in the whole space, Comm. Partial Differential Equations 16 (1991), no. 4-5, 801-820.

[51] H. Choe and B. Jin, Weighted estimate of the asymptotic profiles of the Navier-Stokes flow in $\mathbb{R}^{n}$, J. Math. Anal. Appl. 344 (2008), no. 1, 353-366.

[52] P. Constantin, A.. Majda, and E. Tabak, Singular front formation in a model for quasigeostrophic flow, Phys. Fluids 6 (1994), no. 1, 9-11.

[53] P. Constantin and J. Wu, Behavior of solutions of 2D quasi-geostrophic equations, SIAM J. Math. Anal. 30 (1999), no. 5, 937-948.

[54] A. Córdoba and D. Córdoba, A maximum principle applied to quasi-geostrophic equations, Comm. Math. Phys. 249 (2004), no. 3, 511-528.

[55] M. Dai, E. Feireisl, E. Rocca, G. Shimperna, and M. Schonbek, On asymptotic isotropy for a hydrodynamic model of liquid crystals, arXiv:1409.7499 (2014).

[56] M. Dai, J. Qing, and M. Schonbek, Regularity of solutions to the liquid crystals systems in $\mathbb{R}^{2}$ and $\mathbb{R}^{3}$, Nonlinearity 25 (2012), no. 2, 513-532.

[57] Asymptotic behavior of solutions to liquid crystal systems in $R^{3}$, Comm. Partial Differential Equations 37 (2012), no. 12, 2138-2164.

[58] M. Dai and M. Schonbek, Asymptotic behavior of solutions to the liquid crystal system in $H^{m}\left(\mathbb{R}^{3}\right)$, SIAM J. Math. Anal. 46 (2014), no. 5, 3131-3150.

[59] R. Danchin and M. Paicu, Les théorèmes de Leray et de Fujita-Kato pour le système de Boussinesq partiellement visqueux, Bull. Soc. Math. France 136 (2008), no. 2 (French, with English and French summaries).

[60] C. Doering and J. D. Gibbon, Applied analysis of the Navier-Stokes equations, Cambridge Texts in Applied Mathematics, Cambridge University Press, Cambridge, 1995.

[61] S. Dubois, What is a solution to the Navier-Stokes equations?, C. R. Math. Acad. Sci. Paris 335 (2002), no. 1, 27-32 (English, with English and French summaries).

[62] J. L. Ericksen, Continuum theory of nematic liquid crystals, Res Mechanica 21 (1987), 381-392.

[63] Conservation laws for liquid crystals, Trans. Soc. Rheology 5 (1961), 23-34. MR0158610 (28 \#1833)

[64] L. Escauriaza, G. A. Serëgin, and V. Šverák, $L_{3, \infty}$-solutions of Navier-Stokes equations and backward uniqueness, Uspekhi Mat. Nauk 58 (2003), no. 2(350), 3-44 (Russian); English transl., Russian Math. Surveys 58 (2003), no. 2, 211-250. 
[65] L. Escauriaza, G. Serëgin, and V. Šverák, Backward uniqueness for parabolic equations, Arch. Ration. Mech. Anal. 169 (2003), no. 2, 147-157.

[66] R. Farwig, H. Kozono, and H. Sohr, An $L^{q}$-approach to Stokes and Navier-Stokes equations in general domains, Acta Math. 195 (2005), 21-53.

[67] E. Feireisl and M. Schonbek, On the Oberbeck-Boussinesq approximation on unbounded domains, Abel proceedings (2013).

[68] R. Finn, On the exterior stationary problem for the Navier-Stokes equations, and associated perturbation problems, Arch. Rational Mech. Anal. 19 (1965), 363-406. MR0182816 (32 \#298)

[69] - On the steady-state solutions of the Navier-Stokes equations. III, Acta Math. 105 (1961), 197-244. MR0166498 (29 \#3773)

[70] - On steady-state solutions of the Navier-Stokes partial differential equations, Arch. Rational Mech. Anal. 3 (1959), 381-396 (1959). MR0107442 (21 \#6167)

[71] C. Foias and J.-C. Saut, Asymptotic behavior, as $t \rightarrow+\infty$, of solutions of Navier-Stokes equations and nonlinear spectral manifolds, Indiana Univ. Math. J. 33 (1984), no. 3, 459-477.

[72] Y. Fujigaki and T. Miyakawa, Asymptotic profiles of nonstationary incompressible Navier-Stokes flows in $\mathbb{R}^{n}$ and $\mathbb{R}_{+}^{n}$, Sūrikaisekikenkyūsho Kōkyūroku 1225 (2001), 14-33. Mathematical analysis in fluid and gas dynamics (Japanese) (Kyoto, 2000).

[73] - Asymptotic profiles of nonstationary incompressible Navier-Stokes flows in the whole space, SIAM J. Math. Anal. 33 (2001), no. 3, 523-544.

[74] Asymptotic profiles of nonstationary incompressible Navier-Stokes flows in the half-space, Methods Appl. Anal. 8 (2001), no. 1, 121-157.

[75] H. Fujita and T. Kato, On the Navier-Stokes initial value problem. I, Arch. Rational Mech. Anal. 16 (1964), 269-315.

[76] G. Galdi and J Nestupa, Steady-state Navier-Stokes flow around a moving body, Handbook of Mathematical Analysis in Mechanics of Viscous Fluids. Chapter xxx (Springer, ed.)

[77] G. Galdi, An introduction to the mathematical theory of the Navier-Stokes equations. Vol. I, Springer Tracts in Natural Philosophy, vol. 38, Springer-Verlag, New York, 1994. Linearized steady problems.

[78] G. Galdi, J. Heywood, and Y. Shibata, On the global existence and convergence to steady state of Navier-Stokes flow past an obstacle that is started from rest, Arch. Rational Mech. Anal. 138 (1997), no. 4, 307-318.

[79] G. Galdi and P. Maremonti, Monotonic decreasing and asymptotic behavior of the kinetic energy for weak solutions of the Navier-Stokes equations in exterior domains, Arch. Rational Mech. Anal. 94 (1986), no. 3, 253-266.

[80] I. Gallagher and F. Planchon, On global infinite energy solutions to the Navier-Stokes equations in two dimensions, Arch. Ration. Mech. Anal. 161 (2002), no. 4, 307-337.

[81] I. Gallagher, D. Iftimie, and F. Planchon, Asymptotics and stability for global solutions to the NavierStokes equations, Ann. Inst. Fourier (Grenoble) 53 (2003), no. 5, 1387-1424.

[82] I. Gallagher, Critical function spaces for well-posedness of the Navier-Stokes initial value problems, Handbook of Mathematical Analysis in Mechanics of Viscous Fluids. Chapter xxx (Springer, ed.)

[83] T. Gallay, Infinite energy solutions of the two-dimensional Navier-Stokes equations (2014).

[84] T. Gallay and Y. Maekawa, Existence and Stability of Viscous Vortices, Handbook of Mathematical Analysis in Mechanics of Viscous Fluids. Chapter xxx (Springer, ed.)

[85] T. Gallay and L. Rodrigues, Sur le temps de vie de la turbulence bidimensionnelle, Ann. Fac. Sci. Toulouse Math. (6) 17 (2008), no. 4, 719-733 (French, with English and French summaries). 
[86] Th. Gallay and C. E. Wayne, Long-time asymptotics of the Navier-Stokes and vorticity equations on $\mathbb{R}^{3}$, R. Soc. Lond. Philos. Trans. Ser. A Math. Phys. Eng. Sci. 360 (2002), no. 1799, 2155-2188. Recent developments in the mathematical theory of water waves (Oberwolfach, 2001).

[87] - Invariant manifolds and the long-time asymptotics of the Navier-Stokes and vorticity equations on $\mathbb{R}^{2}$, Arch. Ration. Mech. Anal. 163 (2002), no. 3, 209-258.

[88] _ Global stability of vortex solutions of the two-dimensional Navier-Stokes equation, Comm. Math. Phys. 255 (2005), no. 1, 97-129.

[89] J Gao, Q. Tao, and Z. Yao, Dynamics of nematic liquid crystal flows: the quasilinear approach, arXiv:1412.0498v2 (2015).

[90] P. Germain, Multipliers, para-multipliers, and weak-strong uniqueness for the Navier-Stokes equations, J. Differential Equations 226 (2006), no. 2, 373-428.

[91] P. Germain, N. Pavlović, and G. Staffilani, Regularity of solutions to the Navier-Stokes equations evolving from small data in $\mathrm{BMO}^{-1}$, Int. Math. Res. Not. IMRN 21 (2007), Art. ID rnm087, 35.

[92] Y. Giga, Solutions for semilinear parabolic equations in $L^{p}$ and regularity of weak solutions of the Navier-Stokes system, J. Differential Equations 62 (1986), no. 2, 186-212.

[93] M-H. Giga, Y. Giga, and Jürgen Saal, Nonlinear partial differential equations, Progress in Nonlinear Differential Equations and their Applications, 79, Birkhäuser Boston, Inc., Boston, MA, 2010. Asymptotic behavior of solutions and self-similar solutions.

[94] Y. Giga and T. Kambe, Large time behavior of the vorticity of two-dimensional viscous flow and its application to vortex formation, Comm. Math. Phys. 117 (1988), no. 4, 549-568.

[95] Y. Giga, S. Matsui, and O. Sawada, Global existence of two-dimensional Navier-Stokes flow with nondecaying initial velocity, J. Math. Fluid Mech. 3 (2001), no. 3, 302-315.

[96] Y. Giga and T. Miyakawa, Navier-Stokes flow in $\mathbb{R}^{3}$ with measures as initial vorticity and Morrey spaces, Comm. Partial Differential Equations 14 (1989), no. 5, 577-618.

[97] Y. Giga, T. Miyakawa, and H. Osada, Two-dimensional Navier-Stokes flow with measures as initial vorticity, Arch. Rational Mech. Anal. 104 (1988), no. 3, 223-250.

[98] P. Han, Algebraic $L^{2}$ decay for weak solutions of a viscous Boussinesq system in exterior domains, J. Differential Equations 252 (2012), no. 12, 6306-6323.

[99] P. Han and M. Schonbek, Large time decay properties of solutions to a viscous Boussinesq system in a half space, Adv. Differential Equations 19 (2014), no. 1-2, 87-132. MR3161657

[100] P. Han, Decay results of higher-order norms for the Navier-Stokes flows in $3 D$ exterior domains, Comm. Math. Phys. 334 (2015), no. 1, 397-432.

[101] C. He and T. Miyakawa, On $L^{1}$-summability and asymptotic profiles for smooth solutions to NavierStokes equations in a 3D exterior domain, Math. Z. 245 (2003), no. 2, 387-417.

[102] _ Non-stationary Navier-Stokes flows in a two-dimensional exterior domain with rotational symmetries, Indiana Univ. Math. J. 55 (2006), no. 5, 1483-1555.

[103] _ On weighted-norm estimates for non-stationary incompressible Navier-Stokes flows in a 3D exterior domain, J. Differential Equations 246 (2009), no. 6, 2355-2386.

[104] C He and Z. Xin, On the decay properties of solutions to the non-stationary Navier-Stokes equations in $\mathbb{R}^{3}$, Proc. Roy. Soc. Edinburgh Sect. A 131 (2001), no. 3, 597-619.

[105] J. Heywood, The Navier-Stokes equations: on the existence, regularity and decay of solutions, Indiana Univ. Math. J. 29 (1980), no. 5, 639-681.

[106] - The exterior nonstationary problem for the Navier-Stokes equations, Acta Math. 129 (1972), no. 1-2, 11-34. MR0609550 (58 \#29432)

[107] _ On stationary solutions of the Navier-Stokes equations as limits of nonstationary solutions, Arch. Rational Mech. Anal. 37 (1970), 48-60. MR0412639 (54 \#761) 
[108] M. Hieber, M Nesensohn, J. Prüss, and K Schade, Dynamics of nematic liquid crystal flows: the quasilinear approach, arXiv:1302.4596v1 (2013).

[109] M. Hieber and J. Prüss, Thermodynamical consistent modeling and analysis of nematic liquid crystal flows, arXiv:1504.01237vi (2015).

[110] M Hieber and P Prüss, Handbook of Mathematical Analysis in Mechanics of Viscous Fluids: Modeling and analysis of the Ericksen-Leslie equations for nematic liquid crystal flows. Chapter xxx (Springer, ed.)

[111] M Hieber and J Saal, The Stokes equation in the $L^{p}$-setting: Well-posedness and regularity properties, Handbook of Mathematical Analysis in Mechanics of Viscous Fluids. Chapter xxx (Springer, ed.)

[112] T. Hishida, Lack of uniformity of $L^{2}$ decay for viscous incompressible flows in exterior domains, Adv. Math. Sci. Appl. 2 (1993), no. 2, 345-367. MR1239264 (94j:35144)

[113] — Stationary Navier-Stokes flow in exterior domains and Landau solutions, Handbook of Mathematical Analysis in Mechanics of Viscous Fluids. Chapter xxx (Springer, ed.)

[114] Toshiaki Hishida and Maria Schonbek, Stability of time-dependent Navier-Stokes flow and algebraic energy decay, arXiv:1412.0204 (2014).

[115] J. Huang and M. Paicu, Decay estimates of global solution to 2D incompressible Navier-Stokes equations with variable viscosity, Discrete Contin. Dyn. Syst. 34 (2014), no. 11, 4647-4669.

[116] H. Jia, V. Šverák, and T.-P. Tsai, Self-similar solutions to the non-stationary Navier-Stokes equations, Handbook of Mathematical Analysis in Mechanics of Viscous Fluids. Chapter xxx.

[117] N. Ju, The maximum principle and the global attractor for the dissipative $2 D$ quasi-geostrophic equations, Comm. Math. Phys. 255 (2005), no. 1, 161-181.

[118] R. Kajikiya and T. Miyakawa, On $L^{2}$ decay of weak solutions of the Navier-Stokes equations in $\mathbf{R}^{n}$, Math. Z. 192 (1986), no. 1, 135-148.

[119] G. Karch and D. Pilarczyk, Asymptotic stability of Landau solutions to Navier-Stokes system, Arch. Ration. Mech. Anal. 202 (2011), no. 1, 115-131.

[120] G. Karch, D. Pilarczyk, and M. Schonbek, $A L^{2}$-asymptotic stability of singular solutions to the Navier-Stokes system of equations in $\mathbb{R}^{3}$, arXiv:1308.6667.

[121] J. Kato, The uniqueness of non-decaying solutions for the Navier-Stokes equations, Arch. Ration. Mech. Anal. 169 (2003), no. 2, 159-175.

[122] T. Kato, Strong $L^{p}$-solutions of the Navier-Stokes equation in $\mathbf{R}^{m}$, with applications to weak solutions, Math. Z. 187 (1984), no. 4, 471-480.

[123] G. Knightly, On a class of global solutions of the Navier-Stokes equations, Arch. Rational Mech. Anal. 21 (1966), 211-245. MR0191213 (32 \#8621)

[124] _ A Cauchy problem for the Navier-Stokes equations in $R^{n}$, SIAM J. Math. Anal. 3 (1972), 506-511. MR0312093 (47 \#655)

[125] Some decay properties of solutions of the Navier-Stokes equations, Approximation methods for Navier-Stokes problems (Proc. Sympos., Univ. Paderborn, Paderborn, 1979), Lecture Notes in Math., vol. 771, Springer, Berlin, 1980, pp. 287-298. MR566003 (81c:35104)

[126] H. Koch and D. Tataru, Well-posedness for the Navier-Stokes equations, Adv. Math. 157 (2001), no. $1,22-35$.

[127] L. Kosloff and T. Schonbek, On the Laplacian and fractional Laplacian in an exterior domain, Adv. Differential Equations 17 (2012), no. 1-2, 173-200. MR2906733

[128] _ Existence and decay of solutions of the 2D QG equation in the presence of an obstacle, Discrete Contin. Dyn. Syst. Ser. S 7 (2014), no. 5, 1025-1043.

[129] H. Kozono, $L^{1}$-solutions of the Navier-Stokes equations in exterior domains, Math. Ann. 312 (1998), no. $2,319-340$. 
[130] H. Kozono and T. Ogawa, Two-dimensional Navier-Stokes flow in unbounded domains, Math. Ann. 297 (1993), no. 1, 1-31.

[131] _ Decay properties of strong solutions for the Navier-Stokes equations in two-dimensional unbounded domains, Arch. Rational Mech. Anal. 122 (1993), no. 1, 1-17.

[132] _ On stability of Navier-Stokes flows in exterior domains, Arch. Rational Mech. Anal. 128 (1994), no. 1, 1-31.

[133] H. Kozono and M. Yamazaki, Local and global unique solvability of the Navier-Stokes exterior problem with Cauchy data in the space $L^{n, \infty}$, Houston J. Math. 21 (1995), no. 4, 755-799.

[134] - The stability of small stationary solutions in Morrey spaces of the Navier-Stokes equation, Indiana Univ. Math. J. 44 (1995), no. 4, 1307-1336.

[135] _ On a larger class of stable solutions to the Navier-Stokes equations in exterior domains, Math. Z. 228 (1998), no. 4, 751-785.

[136] I. Kukavica, Space-time decay for solutions of the Navier-Stokes equations, Indiana Univ. Math. J. 50 (2001), no. Special Issue, 205-222. Dedicated to Professors Ciprian Foias and Roger Temam (Bloomington, IN, 2000).

[137] - On the weighted decay for solutions of the Navier-Stokes system, Nonlinear Anal. 70 (2009), no. $6,2466-2470$.

[138] I. Kukavica and Ednei Reis, Asymptotic expansion for solutions of the Navier-Stokes equations with potential forces, J. Differential Equations 250 (2011), no. 1, 607-622.

[139] I. Kukavica and J. J. Torres, Weighted bounds for the velocity and the vorticity for the Navier-Stokes equations, Nonlinearity 19 (2006), no. 2, 293-303.

[140] _ Weighted $L^{p}$ decay for solutions of the Navier-Stokes equations, Comm. Partial Differential Equations 32 (2007), no. 4-6, 819-831.

[141] O. A. Ladyzhenskaya, The mathematical theory of viscous incompressible flow, Second English edition, revised and enlarged. Translated from the Russian by Richard A. Silverman and John Chu. Mathematics and its Applications, Vol. 2, Gordon and Breach, Science Publishers, New York-LondonParis, 1969. MR0254401 (40 \#7610)

[142] L. Landau, A new exact solution of Navier-Stokes equations, C. R. (Doklady) Acad. Sci. URSS (N.S.) 43 (1944), 286-288. MR0011205 (6,135d)

[143] P. Lemarié-Rieusset, Recent developments in the Navier-Stokes problem, Chapman \& Hall/CRC Research Notes in Mathematics, vol. 431, Chapman \& Hall/CRC, Boca Raton, FL, 2002.

[144] J. Leray, Étude de diverses équations integrales non lineaires et de quelques problèmes que pose l'hydrodynamique, J. Math. Pure Appl. 9 (1933), 1-82.

[145] _ Sur le mouvement d'un liquide visqueux emplissant l'espace, Acta Math. 63 (1934), no. 1, 193-248.

[146] F. Leslie, Some constitutive equations for liquid crystals, Arch. Rational Mech. Anal. 28 (1968), no. 4, 265-283.

[147] _ Theory of flow phenomena in liquid crystals, Vol. 4 G, Brown ed., Academic Press,New York, 1979.

[148] F. Lin and C. Liu, Nonparabolic dissipative systems modeling the flow of liquid crystals, Comm. Pure Appl. Math. 48 (1995), no. 5, 501-537.

[149] P. Lions, Mathematical topics in fluid mechanics. Vol. 1, Oxford Lecture Series in Mathematics and its Applications, vol. 3, The Clarendon Press, Oxford University Press, New York, 1996. Incompressible models; Oxford Science Publications.

[150] S. Liu and X. Xu, Global existence and temporal decay for the nematic liquid crystal flows, J. Math. Anal. Appl. 426 (2015), no. 1, 228-246, DOI 10.1016/j.jmaa.2015.01.001. MR3306371 
[151] Y. Maekawa, On asymptotic stability of global solutions in the weak $L^{2}$ space for the two-dimensional Navier-Stokes equations, Analysis (Berlin) 35 (2015), no. 4, 245-257, DOI 10.1515/anly-2014-1302.

[152] M. Korobkov, K. Pileckas, and R Russo, Solution of Leray's Problem for Stationary Navier-Stokes Equations in Plane and Axially Symmetric Spatial Domains, Handbook of Mathematical Analysis in Mechanics of Viscous Fluids. Chapter xxx (Springer, ed.)

[153] P. Maremonti, Stabilita asintotica in media per moti fluidi viscosi in domini esterni, Anna. Mat. Pura Appl. 4 (1986), no. 142, 57-75.

[154] P. Maremonti, On the asymptotic behaviour of the $L^{2}$-norm of suitable weak solutions to the NavierStokes equations in three-dimensional exterior domains, Comm. Math. Phys. 118 (1988), no. 3, 385-400. MR958803 (89k:35185)

[155] K.T. Masuda, On the stability of incompressible viscous fluid motions past objects, J. Math. Soc. Japan 27 (1975), 294-327.

[156] K. Masuda, Weak solutions of Navier-Stokes equations, Tohoku Math. J. (2) 36 (1984), no. 4, 623646.

[157] Y. Meyer, Wavelets, paraproducts, and Navier-Stokes equations, Current developments in mathematics, 1996 (Cambridge, MA), Int. Press, Boston, MA, 1997, pp. 105-212.

[158] T. Miyakawa, On $L^{1}$-stability of stationary Navier-Stokes flows in $\mathbb{R}^{n}$, J. Math. Sci. Univ. Tokyo 4 (1997), no. 1, 67-119. MR1451304 (98f:35119)

[159] _ On uniqueness of steady Navier-Stokes flows in an exterior domain, Adv. Math. Sci. Appl. 5 (1995), no. 2, 411-420. MR1360998 (97f:35164)

[160] _ Application of Hardy space techniques to the time-decay problem for incompressible NavierStokes flows in $\mathbf{R}^{n}$, Funkcial. Ekvac. 41 (1998), no. 3, 383-434.

[161] _ On space-time decay properties of nonstationary incompressible Navier-Stokes flows in $\mathbf{R}^{n}$, Funkcial. Ekvac. 43 (2000), no. 3, 541-557.

[162] _ Notes on space-time decay properties of nonstationary incompressible Navier-Stokes flows in $\mathbb{R}^{n}$, Funkcial. Ekvac. 45 (2002), no. 2, 271-289.

[163] _ On upper and lower bounds of rates of decay for nonstationary Navier-Stokes flows in the whole space, Hiroshima Math. J. 32 (2002), no. 3, 431-462.

[164] T. Miyakawa and M. E. Schonbek, On optimal decay rates for weak solutions to the Navier-Stokes equations in $\mathbb{R}^{n}$, Proceedings of Partial Differential Equations and Applications (Olomouc, 1999), 2001, pp. 443-455.

[165] T. Miyakawa and Hermann Sohr, On energy inequality, smoothness and large time behavior in $L^{2}$ for weak solutions of the Navier-Stokes equations in exterior domains, Math. Z. 199 (1988), no. 4, 455-478.

[166] M. Monniaux and Z. Shen, Hanbook of mathematical fluid mechanics. Chapter xxx, Springer.

[167] Š. Nečasová and P. Rabier, On the time decay of the solutions of the Navier-Stokes system, J. Math. Fluid Mech. 9 (2007), no. 4, 517-532.

[168] C. Niche and María E. Schonbek, Decay of weak solutions to the 2D dissipative quasi-geostrophic equation, Comm. Math. Phys. 276 (2007), no. 1, 93-115.

[169] C. Niche and M. E. Schonbek, Decay characterization of solutions to dissipative equations, J. London Math. Soc. 9 (2015), no. 2, 573-595.

[170] _ Comparison of decay of solutions to two compressible approximations to Navier-Stokes equations, arXiv:1501.. 9 (2015).

[171] T. Ogawa, S. Rajopadhye, and M. Schonbek, Energy decay for a weak solution of the Navier-Stokes equation with slowly varying external forces, J. Funct. Anal. 144 (1997), no. 2, 325-358. 
[172] M. Oliver and E. Titi, Remark on the rate of decay of higher order derivatives for solutions to the Navier-Stokes equations in $\mathbf{R}^{n}$, J. Funct. Anal. 172 (2000), no. 1, 1-18.

[173] C. Oseen, Neuere Methoden und Ergebnisse in der Hydrodynamik, Akademische Verlagsgesellschaft, M.B.H., Leipzig, 1927.

[174] T. Phan and N. Phuc, Stationary Navier-Stokes equations with critically singular external forces: existence and stability results, Adv. Math. 241 (2013), 137-161.

[175] J. Pedlosky, Geophysical Fluid Dynamics, Springer Verlag, New York, 1987.

[176] F. Planchon, Asymptotic behavior of global solutions to the Navier-Stokes equations in $\mathbf{R}^{3}$, Rev. Mat. Iberoamericana 14 (1998), no. 1, 71-93.

[177] A. Ramm, Scattering by obstacles, Mathematics and its Applications, vol. 21, D. Reidel Publishing Co., Dordrecht, 1986.

[178] O. Sawada and Y. Taniuchi, A remark on $L^{\infty}$ solutions to the 2-D Navier-Stokes equations, J. Math. Fluid Mech. 9 (2007), no. 4, 533-542.

[179] M. Schonbek, Decay of solutions to parabolic conservation laws, Comm. Partial Differential Equations 5 (1980), no. 5, 449-473.

[180] M. Schonbek, $L^{2}$ decay for weak solutions of the Navier-Stokes equations, Arch. Rational Mech. Anal. 88 (1985), no. 3, 209-222.

[181] M. Schonbek, Large time behaviour of solutions to the Navier-Stokes equations, Comm. Partial Differential Equations 11 (1986), no. 7, 733-763.

[182] M. Schonbek, Lower bounds of rates of decay for solutions to the Navier-Stokes equations, J. Amer. Math. Soc. 4 (1991), no. 3, 423-449.

[183] M. Schonbek, The Fourier splitting method, Advances in geometric analysis and continuum mechanics (Stanford, CA, 1993), Int. Press, Cambridge, MA, 1995, pp. 269-274.

[184] _ Large time behaviour of solutions to the Navier-Stokes equations in $H^{m}$ spaces, Comm. Partial Differential Equations 20 (1995), no. 1-2, 103-117.

[185] _ Navier-Stokes space time decay revisited, Mathematical analysis on the Navier-Stokes equations and related topics, past and future, GAKUTO Internat. Ser. Math. Sci. Appl., vol. 35, Gakkōtosho, Tokyo, 2011, pp. 173-184.

[186] M. Schonbek and Tomas P. Schonbek, On the boundedness and decay of moments of solutions to the Navier-Stokes equations, Adv. Differential Equations 5 (2000), no. 7-9, 861-898.

[187] M. Schonbek, T. Schonbek, and E. Süli, Large-time behaviour of solutions to the magnetohydrodynamics equations, Math. Ann. 304 (1996), no. 4, 717-756.

[188] M. Schonbek and T. Schonbek, Moments and lower bounds in the far-field of solutions to quasigeostrophic flows, Discrete Contin. Dyn. Syst. 13 (2005), no. 5, 1277-1304.

[189] Asymptotic behavior to dissipative quasi-geostrophic flows, SIAM J. Math. Anal. 35 (2003), no. $2,357-375$.

[190] M. Schonbek and M. Wiegner, On the decay of higher-order norms of the solutions of Navier-Stokes equations, Proc. Roy. Soc. Edinburgh Sect. A 126 (1996), no. 3, 677-685.

[191] P. Secchi, On the stationary and nonstationary Navier-Stokes equations in $\mathbf{R}^{n}$, Ann. Mat. Pura Appl. (4) 153 (1988), 293-305.

[192] J. Serrin, On the interior regularity of weak solutions of the Navier-Stokes equations, Arch. Rational Mech. Anal. 9 (1962), 187-195.

[193] L. Simon, Asymptotics for a class of nonlinear evolution equations, with applications to geometric problems, Ann. of Math. (2) 118 (1983), no. 3, 525-571. 
[194] Z. Skalák, The large-time energy concentration in solutions to the Navier-Stokes equations in the frequency space, J. Math. Anal. Appl. 400 (2013), no. 2, 689-709.

[195] _ A note on lower bounds of decay rates for solutions to the Navier-Stokes equations in the norms of Besov spaces, Nonlinear Anal. 97 (2014), 228-233.

[196] - On the characterization of the Navier-Stokes flows with the power-like energy decay, J. Math. Fluid Mech. 16 (2014), no. 3, 431-446.

[197] H. Sohr, The Navier-Stokes equations, Birkhäuser Advanced Texts: Basler Lehrbücher. [Birkhäuser Advanced Texts: Basel Textbooks], Birkhäuser Verlag, Basel, 2001. An elementary functional analytic approach.

[198] P. Stinga and J. Torrea, Extension problem and Harnack's inequality for some fractional operators, Comm. Partial Differential Equations 35 (2010), no. 11, 2092-2122, DOI 10.1080/03605301003735680. MR2754080 (2012c:35456)

[199] S. Takahashi, A weighted equation approach to decay rate estimates for the Navier-Stokes equations, Nonlinear Anal. 37 (1999), no. 6, Ser. A: Theory Methods, 751-789.

[200] R. Temam, Sur la stabilité et la convergence de la méthode des pas fractionnaires, Ann. Mat. Pura Appl. (4) 79 (1968), 191-379 (French).

[201] F. Vigneron, Spatial decay of the velocity field of an incompressible viscous fluid in $\mathbb{R}^{d}$, Nonlinear Anal. 63 (2005), no. 4, 525-549.

[202] C. Wang, Exact solutions of the Navier-Stokes equations - the generalized Beltrami flows, review and extension, Acta Mech 81 (1990), 69-74.

[203] S. Weng, Remarks on asymptotic behaviors of strong solutions to a viscous Boussinesq system, arXiv:1412.8267 (2014).

[204] _ Space-time decay estimates for the incompressible viscous resistive Hall-MHD equations, arXiv:1412.8267 (2014).

[205] M. Wiegner, Decay results for weak solutions of the Navier-Stokes equations on $\mathbf{R}^{n}$, J. London Math. Soc. (2) 35 (1987), no. 2, 303-313.

[206] H. Wu, Long-time behavior for nonlinear hydrodynamic system modeling the nematic liquid crystal flows, Discrete Contin. Dyn. Syst. 26 (2010), no. 1, 379-396.

[207] M. Yamazaki, The Navier-Stokes equations in the weak- $L^{n}$ space with time-dependent external force, Math. Ann. 317 (2000), no. 4, 635-675.

[208] S. Zelik, Infinite energy solutions for damped Navier-Stokes equations in $\mathbb{R}^{2}$, J. Math. Fluid Mech. 15 (2013), no. 4, 717-745.

[209] L. Zhang, Sharp rate of decay of solutions to 2-dimensional Navier-Stokes equations, Comm. Partial Differential Equations 20 (1995), no. 1-2, 119-127. 